\title{
Dynamics of vortex Rossby waves in tropical cyclones
}

\author{
by
}

\section{Lidia Nikitina}

\author{
A thesis submitted to \\ the Faculty of Graduate and Postdoctoral Affairs \\ in partial fulfillment of \\ the requirements for the degree of \\ Doctor of Philosophy \\ in \\ Mathematics \\ Carleton University \\ Ottawa, Ontario \\ (C) 2013 \\ Lidia Nikitina
}


Library and Archives

Canada

Published Heritage

Branch

395 Wellington Street

Ottawa ON K1A ON4

Canada
Bibliothèque et

Archives Canada

Direction du

Patrimoine de l'édition

395 , rue Wellington

Ottawa ON K1A ON4

Canada
Your file Votre référence

ISBN: 978-0-494-94543-8

Our file Notre référence

ISBN: $978-0-494-94543-8$
NOTICE:

The author has granted a nonexclusive license allowing Library and Archives Canada to reproduce, publish, archive, preserve, conserve, communicate to the public by telecommunication or on the Internet, loan, distrbute and sell theses worldwide, for commercial or noncommercial purposes, in microform, paper, electronic and/or any other formats.

The author retains copyright ownership and moral rights in this thesis. Neither the thesis nor substantial extracts from it may be printed or otherwise reproduced without the author's permission.
AVIS:

L'auteur a accordé une licence non exclusive permettant à la Bibliothèque et Archives Canada de reproduire, publier, archiver, sauvegarder, conserver, transmettre au public par télécommunication ou par l'Internet, prêter, distribuer et vendre des thèses partout dans le monde, à des fins commerciales ou autres, sur support microforme, papier, électronique et/ou autres formats.

L'auteur conserve la propriété du droit d'auteur et des droits moraux qui protege cette thèse. $\mathrm{Ni}$ la thèse ni des extraits substantiels de celle-ci ne doivent être imprimés ou autrement reproduits sans son autorisation.
In compliance with the Canadian Privacy Act some supporting forms may have been removed from this thesis.

While these forms may be included in the document page count, their removal does not represent any loss of content from the thesis.
Conformément à la loi canadienne sur la protection de la vie privée, quelques formulaires secondaires ont été enlevés de cette thèse.

Bien que ces formulaires aient inclus dans la pagination, il n'y aura aucun contenu manquant. 


\begin{abstract}
This thesis describes an analytical study of vortex Rossby waves in tropical cyclones. Observational analyses of hurricanes in the tropical atmosphere indicate the existence of spiral rainbands which propagate outwards from the eye and affect the structure and intensity of the hurricane. These disturbances may be described as vortex Rossby waves.

The aim of this research is to study the propagation of vortex Rossby waves in tropical cyclones and wave-mean-flow interactions near the critical radius where the mean flow angular velocity matches the phase speed of the waves. Depending on the wave magnitude, the problem can be linear or nonlinear. Analytical techniques including Laplace transforms, multiple scaling and asymptotic expansions are used to obtain approximate solutions of the governing linear and nonlinear equations. In this study we carry out asymptotic analyses to examine the evolution of the interactions near the critical radius in some two-dimensional configurations on an $f$-plane and a $\beta$-plane.

The results are used to explain some features of the tropical cyclone's development, namely, the change of angular wind in the critical layer, the secondary eyewall formation and the eyewall dynamics.
\end{abstract}




\section{Acknowledgements}

I would like to express my deepest gratitude to my supervisor, Prof. Lucy Campbell, and thank her for her permanent support and patience, for her wisdom and kindness, for her high-level of professionalism both in research and teaching, for the great time I spent at the School of Mathematics and Statistics during the course of my thesis work.

I appreciate the time and effort the external examiner Prof. Simon Clarke and the committee members Prof. Dave Amundsen, Prof. Arian Novruzi, and Prof. Abhijit Sarkar put into reading and reviewing my thesis in spite of their busy schedules.

I would like to thank Dr. Gilbert Brunet for bringing this interesting problem to our attention. I also would like to thank him for helpful discussions.

This thesis project was supported by Natural Sciences and Engineering Council of Canada (NSERC) for three years with Alexander Graham Bell Canada Graduate Scholarship. 


\section{Contents}

Title page

$\begin{array}{ll}\text { Abstract } & \text { ii }\end{array}$

Acknowledgments $\quad$ iii

List of Tables vi

List of Figures vii

List of Appendices viii

1 Introduction 1

1.1 Motivation for the study and overview of the thesis . . . . . . 1

1.2 Study of tropical cyclones . . . . . . . . . . . . . . 4

2 Basic concepts of geophysical fluid dynamics 13

2.1 Basic equations of fluid mechanics . . . . . . . . . . . . . 14

2.2 Effects of rotation . . . . . . . . . . . . . 16

2.3 The Coriolis effect for a thin layer on a rotating sphere . . . . . . . . 21

2.4 Waves in the ocean and the atmosphere ........... 23 
3 Vortex dynamics in the atmosphere $\quad 26$

3.1 Barotropic vorticity equation . . . . . . . . . . . . . 26

3.2 Barotropic Rossby waves . . . . . . . . . . . . . . . . 34

3.3 Vortex Rossby waves . . . . . . . . . . . . . . 36

3.4 Previous theoretical studies of tropical cyclone dynamics . . . . . . . . 39

4 Steady linear problem $\quad 46$

4.1 Steady linear solution for some special cases . . . . . . . . . . . 46

5 The linear time-dependent problem $\quad 64$

5.1 Laplace transform of the linear time-dependent equation . . . . . . . 65

5.2 Evaluation of the inverse Laplace transform . . . . . . . . . . . 75

5.3 Time-dependent solution close to the critical layer . . . . . . . . . 88

5.4 The inner layer solution . . . . . . . . . . . . . . 91

5.5 Discussion of the linear time-dependent solution . . . . . . . . . . . 94

$6 \quad$ Effect of nonlinearity and $\beta$-effect $\quad 97$

6.1 Weakly-nonlinear analysis . . . . . . . . . . . . . 97

6.2 Effect of the variation of the Coriolis force . . . . . . . . . . . . 101

6.3 Combined effects of nonlinearity and the $\beta$-effect . . . . . . . . . . 109

6.4 Evolution of the mean flow in the inner layer . . . . . . . . . . . 114

7 Conclusions $\quad 118$

7.1 Summary . . . . . . . . . . . . . . . . . . . . 118

A Hypergeometric functions $\quad 122$

A.1 Generalized hypergeometric functions . . . . . . . . . . . . . 122

A.2 The solutions of the hypergeometric equation $\ldots \ldots \ldots$ 


\section{List of Tables}

6.1 Wavenumbers corresponding to the terms in equation $(6.49) \ldots 111$ 


\section{List of Figures}

1.1 Tropical cyclone structure . . . . . . . . . . . . . . 7

1.2 Hurricane Ivan 2004 , secondary eyewall formation . . . . . . . . . . . 11

2.1 Vector in a rotating frame ............... 17

2.2 Centripetal acceleration . . . . . . . . . . . . . . 19

2.3 Local Cartesian coordinates . . . . . . . . . . . . . . . . . 21

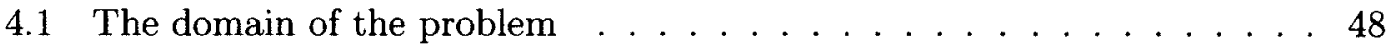

4.2 The angular velocity profile . . . . . . . . . . . . 50

4.3 Intervals for the steady solution . . . . . . . . . . . . . . . 57

4.4 The amplitude of the steady solution. . . . . . . . . . . . 62

4.5 Contour plots for the steady solution . . . . . . . . . . 63

5.1 The contour of integration in the complex $s$-plane. Case 1 and 2. . . 69

5.2 The contour of integration in the complex $s$-plane. Case $3 \ldots \ldots$

6.1 Influence of $\beta$-effect on the vortex Rossby wave propagation . . . . . 106

6.2 Schematic diagrams for wavenumbers . . . . . . . . . . . . 111

6.3 Dynamics of the critical radius location. . . . . . . . . . 117

A.1 The hypergeometric functions. . . . . . . . . . . . 127 


\section{List of Appendices}

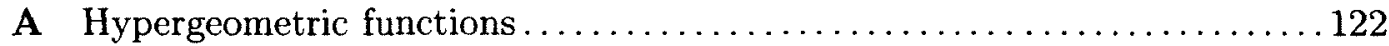

A1 Generalized hypergeometric functions $\ldots \ldots \ldots \ldots \ldots \ldots \ldots \ldots \ldots \ldots \ldots \ldots \ldots$

A2 The solutions of the hypergeometric equation $\ldots \ldots \ldots \ldots \ldots \ldots 123$

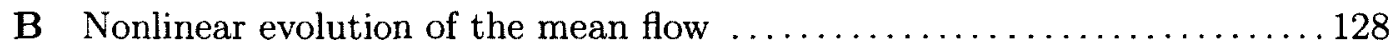

C The phase shift for the steady solution $\ldots \ldots \ldots \ldots \ldots \ldots \ldots \ldots \ldots \ldots \ldots \ldots \ldots$ 


\section{Chapter 1}

\section{Introduction}

\subsection{Motivation for the study and overview of the thesis}

The effect of the Coriolis force gives rise to large scale oscillations in the atmosphere and ocean which are known as Rossby waves. These waves affect weather and climate and play a role in various observed phenomena including the development of hurricanes or tropical cyclones. The dynamics of tropical cyclones have been studied extensively during the past few decades using observational data, theoretical modeling and numerical simulations. The motivation for understanding and simulating hurricanes is based on the profound effects that hurricanes can have on human life as one of the most devastating disasters. But a full theory of hurricane dynamics is far from complete.

Vortex Rossby waves propagating within tropical cyclones are known to play an important part in cyclone development and evolution. The waves affect the strength, structure and dynamics of a cyclone. In particular, vortex Rossby waves are believed 
to play a role in the formation of the secondary eyewall, an outer ring of intense thunderstorms that develops outside the initial eyewall of the hurricane and eventually replaces the original eyewall.

In this thesis we present a simple two dimensional configuration that represents Rossby wave propagation in a cyclonic vortex and describes a possible mechanism for the secondary eyewall formation. Approximate analytic solutions are derived using asymptotic methods and weakly-nonlinear analyses. Previous analytic investigations of this mechanism have been based on linearized equations that did not include the effects of the gradient of the Coriolis force. The addition of nonlinearity and Coriolis effects are important features of this thesis project.

An overview of the thesis is as follows. The rest of this chapter gives background information about tropical cyclones. It includes a description of cyclones and the physical processes involved in the development of the tropical cyclones, as well some basic information about the structure of cyclones, their life cycle, intensity. In particular, the secondary eyewall replacement cycle is described.

Chapter 2 gives a mathematical description of the basic features of geophysical fluid dynamics, including the effect of the Earth's rotation, and a description of Rossby waves.

Chapter 3 deals with analysis of vortex dynamics in the atmosphere. This chapter includes the derivation of the basic equations that are solved in the thesis. These equations describe tropical cyclones as well as the propagation of vortex Rossby waves in tropical cyclones. Chapter 3 also gives information about some basic approaches to the study of the dynamics of tropical cyclones.

Chapters 1-3 present introductory material on hurricanes, geophysical fluid dynamics, and Rossby waves based on standard texts and papers on these topics. The derivation of the basic equations follows Kundu and Cohen (2004), Holton (1992), 
Landau and Lifshitz (1953), and Gill (1982). The equations for the vortex waves are based on the studies of Montgomery and Kallenbach (1997) and Brunet and Montgomery (2002).

The description of my original work starts in Chapter 4. I represent a tropical cyclone as a vortex in a two-dimensional configuration. The vortex Rossby waves are considered as small perturbations to the basic rotation of the cyclone, which are periodic in the azimuthal direction and have amplitude that varies in the radial direction. I derive a solution of the governing equation for a configuration in which the wave amplitude is steady. The waves propagate on an $f$-plane, a horizontal plane in which the Coriolis force is taken to be a constant. The waves are assumed to be of small amplitude and the governing equation is linearized. The problem is defined in terms of polar coordinates. The waves are generated by a boundary condition, periodic both in time and in the azimuthal direction, at some fixed distance from the centre of the vortex.

The steady linear problem is governed by an ordinary differential equation which describes the wave amplitude in terms of the radial variable. The equation is singular at the radius where the cyclone angular velocity is equal to the phase speed of the waves. This is called the critical radius and the region close to this radius is called the critical layer. The solution of the ordinary differential equation is expressed in terms of hypergeometric functions.

In Chapter 5 a Laplace transform is used to derive an approximate time-dependent solution for the linear problem. I derive an approximate solution valid in the outer region, far from the critical layer, as well as in the inner region inside the critical layer.

In Chapter $6 \mathrm{I}$ include the effects of nonlinearity and the " $\beta$-effect" which arises from the latitudinal variation of the Coriolis force. In section 6.1 I carry out a weaklynonlinear analysis of the nonlinear equation considering the nondimensional wave 
amplitude $\varepsilon$ as a small parameter without taking into account the $\beta$-effect. In section 6.2 the $\beta$-effect is included in the linear problern and in section 6.3 I discuss the effect of including both nonlinearity and the $\beta$-effect. The nonlinear interaction between vortex waves and the mean flow changes the angular momentum and changes the mean flow speed in the inner layer. This is discussed in section 6.4. I also give some explanation for the secondary eyewall formation and the eyewall replacement cycle.

Appendix A describes the properties of the hypergeometric equation, its singular points, and its solutions that I use in my thesis. Appendix B gives the derivation of the angular momentum equation that $I$ use in Chapter 6 to examine the nonlinear evolution of the angular velocity of the mean flow. Appendix $\mathrm{C}$ explains the phase shift of a singular term in the steady solution.

\subsection{Study of tropical cyclones}

A cyclone is a system of rotating fluid flow with sustained winds in the form of an axisymmetric vortex. In this section a brief description of tropical cyclones, their development and their structure is given following Holton (1992), Ogawa (1992) and the review article of Emanuel (2003). The eyewall replacement cycle is described.

\section{Tropical cyclones}

A tropical cyclone is a cyclone that originates over tropical oceans and is driven by heat transfer from the ocean. Tropical cyclones are categorized according to their maximum wind speed, defined as the maximum speed of the wind at an altitude of $10 \mathrm{~m}$. Tropical cyclones with maximum wind speeds in the range $15-17 \mathrm{~ms}^{-1}$, are known as tropical depressions; when their speeds are in the range of 18 to $32 \mathrm{~ms}^{-1}$, they are called tropical storms. Tropical cyclones with maximum winds of $33 \mathrm{~ms}^{-1}$ or 
greater are called hurricanes in the western North Atlantic and eastern North Pacific regions; in the South Pacific and Indian Oceans they are usually called cyclones; in East Asia, e.g. Japan and China, they are usually called typhoons. Other names used include "cordonazo" in Mexico, "bagyo" in the Philippines, and "tainos" in Haiti.

The kinetic energy of these large scale vortex motions is about $10^{18}$ Joules. Tropical cyclones are among the most spectacular and deadly geophysical phenomena. Tropical cyclones that cause extreme destruction are rare, although when they occur, they can cause great amounts of damage or thousands of fatalities. The 1970 Bhola cyclone is the deadliest tropical cyclone on record, killing more than 300,000 people and potentially as many as 1 million after striking the densely populated Ganges Delta region of Bangladesh on 13 November 1970. The North Indian cyclone basin has historically been the deadliest. The Great Hurricane of 1780 is the deadliest Atlantic hurricane on record, killing about 22,000 people in the Lesser Antilles in the Caribbean. A tropical cyclone does not need to be extremely strong to cause memorable damage; deaths can be caused by rainfall, flood, or mudslides. The Galveston Hurricane of 1900 is the deadliest natural disaster in the United States, killing an estimated 6,000 to 12,000 people in Galveston, Texas. Hurricane Katrina is estimated as the costliest tropical cyclone worldwide, causing $\$ 81.2$ billion in property damage with overall damage estimates exceeding $\$ 100$ billion. Katrina killed at least 1,836 people after striking Louisiana and Mississippi as a major hurricane in August 2005. Damage from Hurricane Sandy in 2012 is estimated as $\$ 50$ billion. Hurricane Andrew (1992) is also one of the most destructive tropical cyclones in U.S history, with damages totaling $\$ 40.7$ billion.

Up until World War II, detection of tropical cyclones relied on reports from coastal stations, islands, and ships at sea. At this time, it is likely that many storms escaped detection entirely, and some of them were observed only once or a few times during 
the course of their life cycle. During World War II, military aircraft were tasked with finding tropical cyclones that could pose a danger to naval operations. An aircraft was able to penetrate the interior of strong storms for the first time in July 27, 1943, flying into the eye of a Gulf of Mexico hurricane. At about the same time, the first radar images revealed the structure of tropical cyclones including the eye and spiral rainbands. A great step forward came in 1960, when the first image of a tropical cyclone was transmitted from a polar orbiting satellite. Now all tropical cyclones are recorded by satellite based measurements.

\section{The structure of a tropical cyclone}

The radius of a tropical cyclone is generally between 100 and $2000 \mathrm{~km}$. A cyclone of high intensity tends to develop an eye, an area of relative calm (and lowest atmospheric pressure) at the center of circulation. The eye is often visible in satellite images as a small, circular, cloud-free spot. Surrounding the eye is the eyewall, an area about 16 $\mathrm{km}$ to $80 \mathrm{~km}$ wide in which the strongest thunderstorms and winds circulate around the storm's center. The maximum sustained winds in the strongest tropical cyclones have been estimated at about $85 \mathrm{~ms}^{-1}$. Maximum upflow occurs in the eyewall. In intense storms, clouds over the eyewall may form a nearly concentric ring. There may be two or even three eyewalls present at the same time, evolving through a characteristic cycle in which the outer eyewalls contract inward and replace dissipating inner eyewalls.

The earliest radar images of tropical cyclones showed the outward-propagating spiral rainbands that accompany them. They consist of bands of cumulus clouds, some tens of kilometers wide with radial propagation speeds of about $4 \mathrm{~ms}^{-1}$, sometimes connecting with the eyewall. Outside the eyewalls the wind speed and intensity decrease gradually at first and then fall off faster at large radius.

Tropical cyclones are formed in four major phases. The first stage occurs when a 


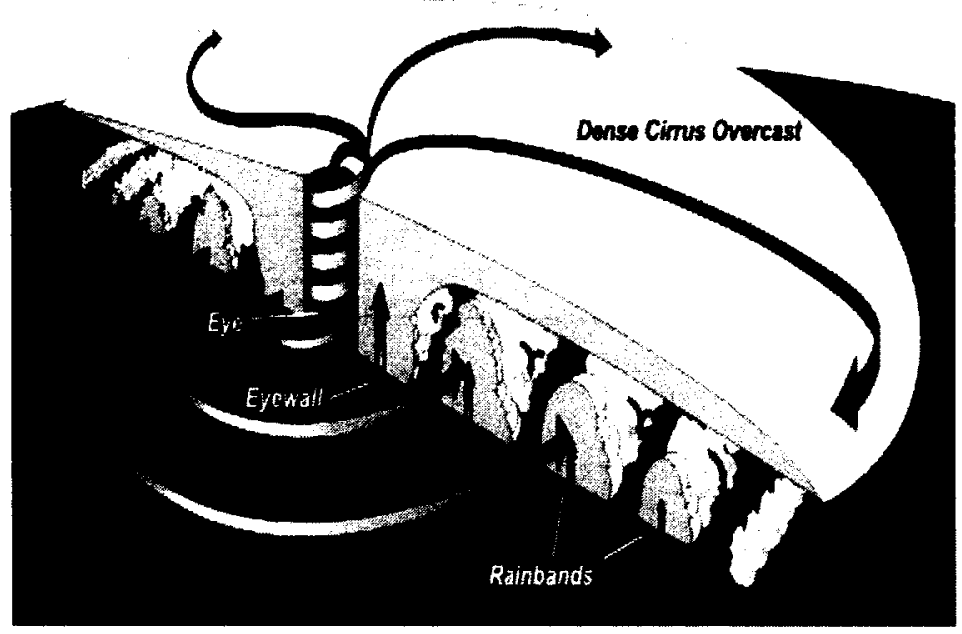

Figure 1.1: Tropical cyclone structure. The main parts of a tropical cyclone are the eye, the eyewall, and the rainbands. This figure is taken from http://serc.carleton.edu/research education/katrina/understanding.html.

collection of small thunderstorms forms over the tropical ocean. The hurricanes that threaten the East and Gulf coasts of the United States, for example, are the product of storms that first form over the coast of western Africa and head west across the Atlantic Ocean. The cyclones that strike East Asia and Australia form over the central Pacific. Australia is also affected by cyclones which form in the North-Western Indian Ocean and Torres Strait. Tropical cyclones are formed between $5^{\circ}$ and $20^{\circ}$ latitude, because of the high humidity, light winds, and warm sea surface temperatures present in these locations. Cyclones and hurricanes can only form when the ocean water is around $26.5^{\circ} \mathrm{C}$ or higher, and these conditions are present in these latitudes during certain months of the year.

Weather conditions in the central Atlantic and Pacific Oceans are most favorable for hurricane formation during the summer and early fall months, which is why the period of June to October is often referred to as "Hurricane Season", in the north- 
ern hemisphere. The opposite situation occurs in the southern hemisphere since the tropical waters of Australia are warmest from December to April.

A tropical disturbance officially enters the second stage of development called a tropical depression once the sustained wind speed within the storm reaches $15 \mathrm{~ms}^{-1}$. The term tropical depression refers to the falling surface pressures measured in the region surrounding the storm. The pressure drop occurs as water vapor within the storm condenses into water droplets and releases latent heat into the atmosphere. Latent heat is defined as the energy released when a substance changes phases, such as gas to liquid in this case.

This process becomes a chain reaction that pulls hot, humid air from the surface of the ocean up to high altitude where the air becomes cold and water vapor condenses into thick clouds. This growing air mass becomes increasingly dense causing the atmospheric pressure to grow. The increasing pressure pushes the growing mass of clouds outward away from the center to create the spiraling bands of clouds that hurricanes are known for. As the air mass spirals outward, its pressure decreases and the dense air plunges back towards the ocean surface where it started. It now picks up vapor again from the warm waters below and is sucked back into the center of the depression to begin its journey anew. As the cycle continues, the surface pressure at the center drops lower, causing the circulation of the air to strengthen and the winds to grow increasingly stronger.

Once the sustained wind speed increases to $18 \mathrm{~ms}^{-1}$, the tropical depression enters the third stage of development called a tropical storm. Until now, the storm has not had any formal designation. Tropical depressions are usually numbered in a sequential order as they appear, but it is not until they become tropical storms that they receive an official name. The tradition of naming storms dates back to the arrival of the Europeans in the Americas, but the current method of systematically naming tropical 
storms was not formalized until 1953. Female names were used exclusively until the late 1970s when the use of both male and female names in an alternating format was adopted.

It is also during the tropical storm phase that the storm begins to take on its characteristic appearance as a rotating spiral with bands of clouds circulating around the point of lowest surface pressure at its center.

When the wind speed reaches $33 \mathrm{~ms}^{-1}$, the tropical storm is officially classified as a tropical cyclone or hurricane. This is the fourth stage of the hurricane development. The storm now develops an eye at the center of the circulating spirals where there is very little wind, few clouds, and extremely low atmospheric pressure. The eye may extend from 8 to $65 \mathrm{~km}$ in diameter. Surrounding the eye is an area called the eyewall that circulates around the eye and contains the highest clouds. The eyewall is the region containing the strongest winds, heaviest precipitation, and most intense thunderstorms of any part of the hurricane. The most destructive region of the cyclone is the portion of the eyewall rotating in the direction of the forward motion of the storm since the force of the circulating winds is combined with the motion of the storm. Radiating outward from the center are the spiraling bands of clouds that produce heavy rain and wind but are still considerably weaker than the eyewall.

Cyclones typically move westward at a speed of about $4 \mathrm{~ms}^{-1}$ during the early stages of formation. The storms are steered in this direction by the trade winds that occur near the equator and blow towards the west. As a storm moves further from the equator, its course is turned towards the poles and the cyclone curves towards the north in the northern hemisphere or towards the south in the southern hemisphere. Tropical storms that form in the northern hemisphere always rotate in a counterclockwise direction due to the Coriolis force induced by the Earth's rotation, while tropical storms of the southern hemisphere rotate clockwise. 


\section{Eyewall replacement cycle}

Eyewall replacement cycles occur naturally in intense tropical cyclones. Fortner (1958) was the first to document the concentric eyewall and eyewall replacement cycle in Typhoon Sarah in 1956. When a tropical cyclone reaches its highest intensity, the asymmetric ring of outer rainbands contracts and strengths and organizes into a ring of thunderstorms called an outer eyewall, or secondary eyewall, around the existing inner eyewall (Willoughby et al., 1982). As a result, the inner eyewall weakens and is eventually replaced by the more intense outer eyewall. This process takes place on the order of a day or two (Sitkowski et al., 2011). This process of weakening followed by reintensification is called the eyewall replacement cycle. Recent measurements have shown that nearly half of all tropical cyclones attaining at least $60 \mathrm{~ms}^{-1}$ wind speeds undergo the concentric eyewall replacement cycle (Hawkins and Helveston, 2008). In these hurricanes the asymmetric outer rainbands form their own convective ring (secondary eyewall) in coincidence with a local tangential wind maximum around the inner eyewall (Willoughby et al., 1982). Figure 1.2 shows a radar image of Hurricane Ivan (2004) with a developed secondary eyewall.

The motivation to understand and forecast the eyewall replacement cycle is high, because the process can have very serious consequences arising from dramatic intensity and structure changes. The formation of an outer eyewall can cause a decrease in the maximum wind speed at the location of the initial eyewall, while the outer wind maximum tends to broaden the hurricane wind field which can lead to an increase of integrated kinetic energy (Maclay et al., 2008). This has profound impacts both near and far from coastal regions. A sudden expansion of hurricane force winds near landfall can impact a larger coastal area while reducing preparation time. When a hurricane is farther from shore, an increased hurricane wind field is likely to lead to a greater 


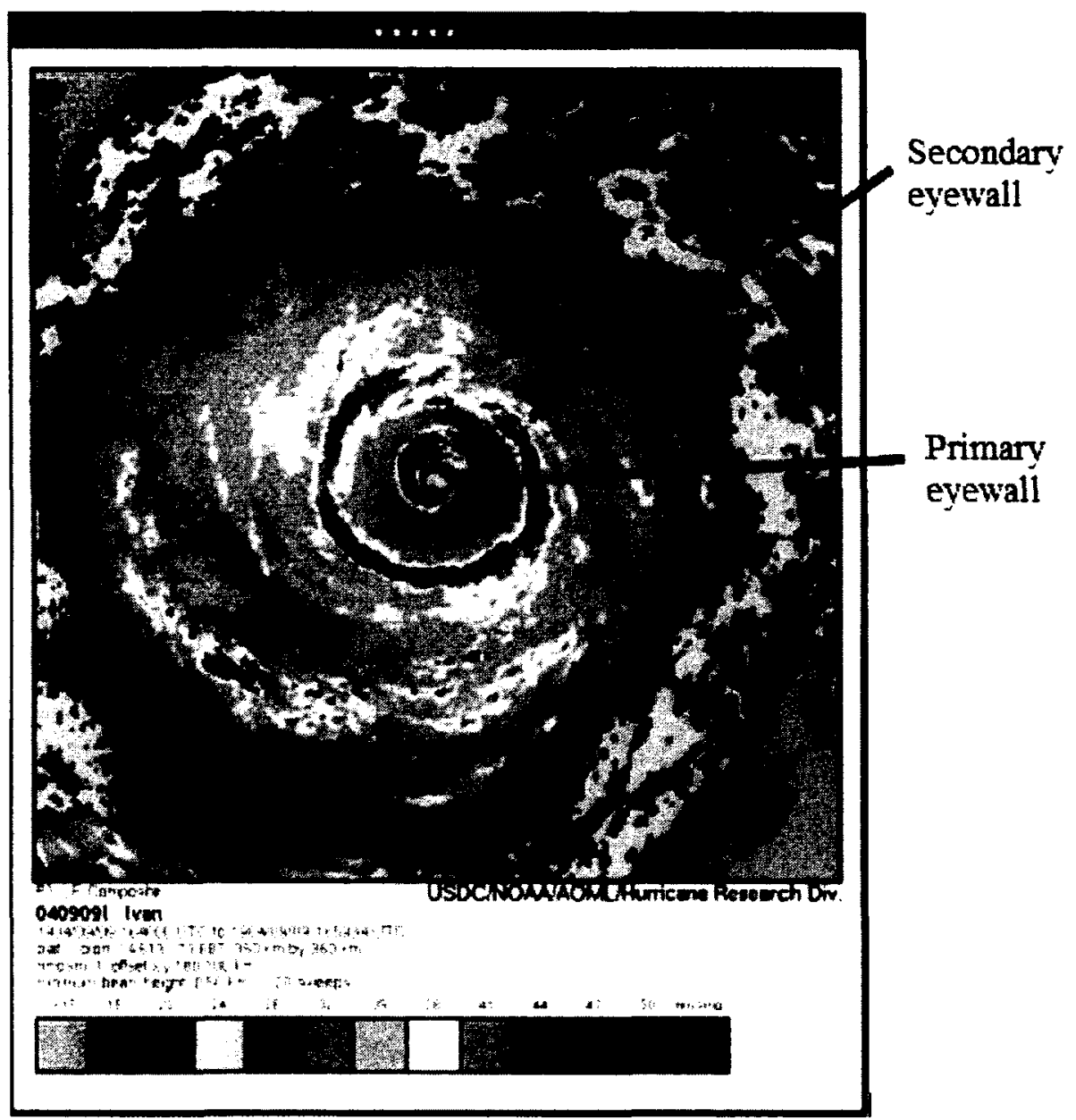

Figure 1.2: Hurricane Ivan. Radar image of Hurricane Ivan (2004) showing the secondary eyewall formation around the primary eyewall.

This image is taken from the website of the NOAA Hurricane Research Department http://www.aoml.noaa.gov/hrd/Storm_pages/ivan2004/i040909i1640_1700c.jpg 
storm (Irish et al., 2008). In addition, the contraction of an outer eyewall near the end of an eye replacement cycle can sometimes lead to rapid intensification resulting in a more intense hurricane than when the process of eyewall formation began. This was the case for Hurricane Andrew (1992), which intensified to a category 5 hurricane as it approached the southeast coast of Florida during the end of an eyewall replacement cycle (Willoughby and Black, 1996). Observations of the hurricane season of 2005 (Houze et al., 2007) showed that the winds in the secondary (or outer) eyewall were initially weaker than those in the original primary (or inner) eyewall, but as the secondary eyewall contracted, the storm reintensified. The processes occurring in the moat region between the old and new eyewalls became dynamically similar to those in the eye. 


\section{Chapter 2}

\section{Basic concepts of geophysical fluid dynamics}

In this chapter I give a basic description of geophysical fluid dynamics and its application to wave and vortex processes in the atmosphere.

The term geophysical fluid dynamics refers to the dynamics of fluid flows, particularly air and water, in the Earth's environment. The discipline includes the study of both fluid phases: liquids (waters in the ocean) and gases (in the Earth's atmosphere and in the atmospheres of other planets). Usually geophysical fluid dynamics deals with large-scale flows, and the rotation of the Earth or density differences (warm and cold air, fresh and saline waters) are important for these processes. Typical problems in geophysical fluid dynamics concern the variability of the atmosphere (weather and climate dynamics), of the ocean (waves, vorticity and currents), the motions in the Earth's interior responsible for the magnetic dynamo effect, vortices on other planets (such as Jupiter's Great Red Spot), and convection in stars (the Sun, in particular).

We live within the atmosphere and are ever affected by the weather and its rather chaotic behaviour. The motion of the atmosphere is connected with that of the ocean, 
with which it exchanges fluxes of momentum, heat and moisture, and it makes the dynamics of the ocean as important as the atmosphere.

The two features that distinguish geophysical fluid dynamics from other areas of fluid dynamics are the rotation of the Earth and the vertical density stratification of a medium because of the vertical gradient of temperature or salinity.

The effect of the rotation of the Earth takes the form of the Coriolis force and gives rise to large scale oscillations in the atmosphere and ocean which are known as Rossby waves. The effects of stratification and the gravitational force give rise to internal gravity waves and surface gravity waves. These waves have profound effects on weather and climate and play a role in various observed phenomena including the development of tropical cyclones. This thesis deals with vortex Rossby waves which propagate in a tropical cyclone and have an influence on the cyclone strength, structure and dynamics.

The motion of fluid in the atmosphere and the ocean is naturally studied in a coordinate frame rotating with the Earth. In this chapter we discuss the basic theory of Rossby waves in a geophysical fluid that is affected by the Coriolis force. We describe these effects in the governing equations following Landau and Lifshitz (1953), Kundu and Cohen (2004), Holton (1992), and Gill (1982).

\subsection{Basic equations of fluid mechanics}

The study of fluid mechanics is based on the laws of conservation of mass, momentum, and energy. 


\section{Conservation of mass}

The conservation of mass equation is

$$
\frac{\partial \rho}{\partial t}+\nabla \cdot(\rho \mathbf{u})=0
$$

where $\rho$ is the density and $\mathbf{u}$ is the fluid velocity. This equation is called the continuity equation and expresses the differential form of the principle of conservation of mass.

\section{Conservation of momentum}

The momentum equation

$$
\rho \frac{D u_{i}}{D t}=-\frac{\partial p}{\partial x_{j}}+\rho g_{i}+\frac{\partial}{\partial x_{j}}\left[\mu\left(\frac{\partial u_{i}}{\partial x_{j}}+\frac{\partial u_{j}}{\partial x_{i}}\right)-\frac{2}{3} \mu \nabla \cdot \mathbf{u} \delta_{i j}\right]
$$

is known as the Navier-Stokes equation. The notation $\frac{D u_{i}}{D t}$ refers to the full derivative,

$$
\frac{D}{D t}=\frac{\partial}{\partial t}+\frac{\partial}{\partial x_{i}} \frac{\partial x_{i}}{\partial t}
$$

Here $p$ is the pressure, $\mathbf{g}$ is the gravity acceleration, $\mu$ is the coefficient of dynamic viscosity, $i$ and $j$ correspond to coordinate axes, the repeated indices mean summation over $i=1,2,3$ or $j=1,2,3$. For an incompressible fluid the momentum equation is written in the form:

$$
\rho \frac{D u_{i}}{D t}=-\frac{\partial p}{\partial x_{i}}+\rho g_{i}+\mu \nabla^{2} u_{i}
$$

or in vector form as

$$
\rho \frac{D \mathbf{u}}{D t}=-\nabla p+\rho \mathbf{g}+\mu \nabla^{2} \mathbf{u}
$$

For atmospheric dynamics the viscous term in the momentum equation is small 
relative to other terms. For hurricane dynamics the length scale is $L \sim 10^{5} \mathrm{~m}$ and the velocity scale is $U \sim 10 \mathrm{~ms}^{-1}$. The kinematic viscosity of the atmosphere is $\nu=\mu / \rho \sim 10^{-5} \mathrm{~m}^{2} \mathrm{~s}^{-1}$. Hence, the Reynolds number which is a measure of the ratio of inertial forces to viscous forces $R e=U L / \nu \sim 10^{11}$ is very large for hurricane-scale flows, and we can neglect the viscous terms in the momentum equation

$$
\rho \frac{D \mathbf{u}}{D t}=-\nabla p+\rho \mathbf{g}
$$

\section{Conservation of energy}

The conservation of energy equation is

$$
\rho \frac{D e}{D t}=-p(\nabla \cdot \mathbf{u})+\phi-\frac{\partial q_{i}}{\partial x_{i}}
$$

where $e$ is the internal energy of the system, vector $\mathbf{q}$ is the heat flux through a unit area, $\phi$ is the rate of viscous dissipation. This is called the thermal energy equation. The set of equations (2.1), (2.2) and (2.7) is used as the starting point for studies of fluid dynamics.

\subsection{Effects of rotation}

In studying the motion of the fluid in the atmosphere we often have to take into account the rotation of the Earth. The Earth's rotation is important when the scales of the processes under consideration are large and comparable with the Earth's radius. Following the description given in Kundu and Cohen (2004) and Holton (1992), we consider a frame of reference $\left(x_{1}, x_{2}, x_{3}\right)$ rotating with a uniform angular velocity $\Omega$ 


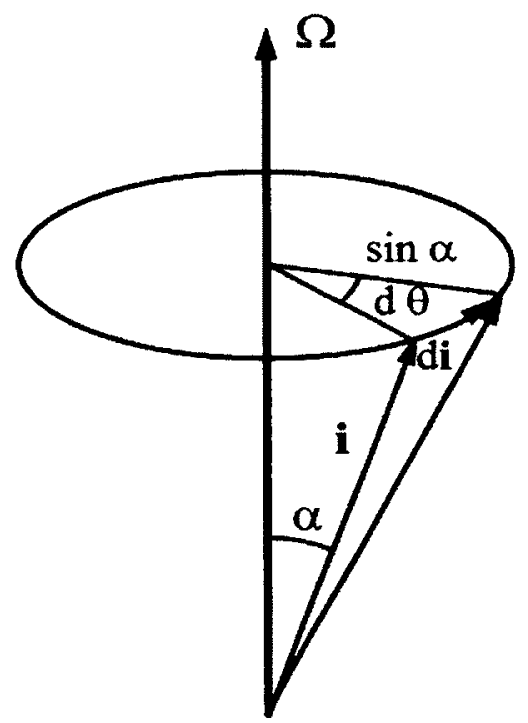

Figure 2.1: Vector in a rotating frame. The vector $\mathrm{i}$ rotates with the reference frame. $\boldsymbol{\Omega}$ is the angular velocity of the frame rotation, $\alpha$ is the angle between the vector $\mathbf{i}$ and the axis of rotation. The change in the angle of rotation $d \theta$ causes the change in the vector di.

with respect to a fixed frame. Any vector $\mathbf{P}$ is represented in the rotating frame by

$$
\mathbf{P}=P_{1} \mathbf{i}_{1}+P_{2} \mathbf{i}_{2}+P_{3} \mathbf{i}_{\mathbf{3}}
$$

To a fixed observer the directions of the rotating unit vectors $\mathbf{i}_{\mathbf{1}}, \mathbf{i}_{\mathbf{2}}$ and $\mathbf{i}_{\mathbf{3}}$ change with time. To this observer the time derivative of $\mathbf{P}$ is

$$
\frac{d \mathbf{P}}{d t}=\frac{d}{d t}\left(P_{1} \mathbf{i}_{\mathbf{1}}+P_{2} \mathbf{i}_{\mathbf{2}}+P_{3} \mathbf{i}_{\mathbf{3}}\right)=\mathbf{i}_{1} \frac{d P_{1}}{d t}+\mathbf{i}_{\mathbf{2}} \frac{d P_{2}}{d t}+\mathbf{i}_{\mathbf{3}} \frac{d P_{3}}{d t}+P_{1} \frac{d \mathbf{i}_{\mathbf{1}}}{d t}+P_{2} \frac{d \mathbf{i}_{\mathbf{2}}}{d t}+P_{3} \frac{d \mathbf{i}_{\mathbf{3}}}{d t},
$$

where we have used the notation $d \mathbf{i}_{\mathbf{j}} / d t, j=1,2,3$, to represent the rates of change of the unit vectors. To an observer in the rotating frame, the rate of change of $\mathbf{P}$ is 
the sum of the first three terms in $(2.9)$, so that

$$
\left(\frac{d \mathbf{P}}{d t}\right)_{F}=\left(\frac{d \mathbf{P}}{d t}\right)_{R}+P_{1} \frac{d \mathbf{i}_{1}}{d t}+P_{2} \frac{d \mathbf{i}_{2}}{d t}+P_{3} \frac{d \mathbf{i}_{\mathbf{3}}}{d t}
$$

where the subscript of $F$ refers to the fixed frame and the subscript of $R$ to the rotating frame.

Each unit vector $\mathrm{i}$ traces a cone with a radius of $\sin \alpha$, where $\alpha$ is a constant angle between the vector and axis of rotation (Figure 2.1). The magnitude of the change of $\mathbf{i}$ in time $d t$ is $|d \mathbf{i}|=\sin \alpha d \theta$, which is the length traveled by the tip of $\mathbf{i}$. The magnitude of the rate of change is therefore $|d \mathbf{i} / d t|=\sin \alpha(d \theta / d t)=|\Omega| \sin \alpha$, and the direction of the rate of change is perpendicular to the $(\Omega, \mathbf{i})$-plane. Thus, $(d \mathbf{i} / d t)=\Omega \times \mathbf{i}$ for any rotating unit vector $\mathbf{i}$. The sum of the last three terms in (2.10) can thus be written as $P_{1} \boldsymbol{\Omega} \times \mathbf{i}_{1}+P_{2} \boldsymbol{\Omega} \times \mathbf{i}_{2}+P_{3} \boldsymbol{\Omega} \times \mathbf{i}_{\mathbf{3}}=\boldsymbol{\Omega} \times \mathbf{P}$. Equation (2.10) then becomes

$$
\left(\frac{d \mathbf{P}}{d t}\right)_{F}=\left(\frac{d \mathbf{P}}{d t}\right)_{R}+\mathbf{\Omega} \times \mathbf{P}
$$

We can use this argument to derive an expression for the Coriolis force. Consider the point in the atmosphere with position vector $\mathbf{r}$. The application of the rule (2.11) to the vector $\mathbf{r}$ gives

$$
\mathbf{u}_{F}=\mathbf{u}_{R}+\Omega \times \mathbf{r}
$$

where $\mathbf{u}_{F}=\left(\frac{d \mathbf{r}}{d t}\right)_{F}$ and $\mathbf{u}_{R}=\left(\frac{d \mathbf{r}}{d t}\right)_{R}$. Applying (2.12) to the velocity vector $\mathbf{u}_{F}$ gives

$$
\left(\frac{d \mathbf{u}_{F}}{d t}\right)_{F}=\left(\frac{d \mathbf{u}_{F}}{d t}\right)_{R}+\Omega \times \mathbf{u}_{F}
$$




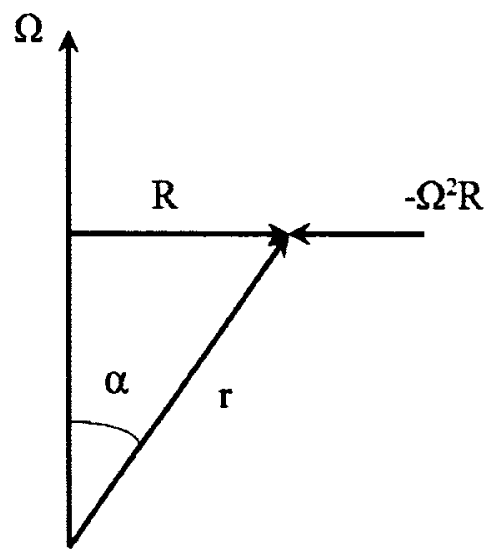

Figure 2.2: Centripetal acceleration. The centripetal acceleration $-\Omega^{2} \mathbf{R}$ is perpendicular to the axis of rotation. $\Omega$ is the angular velocity of the rotating frame, $\mathbf{r}$ is the position vector of a point in the frame, $\alpha$ is the angle between the vector $\bar{r}$ and the axis of rotation, $\mathbf{R}$ is the projection of $\mathbf{r}$ onto the plane perpendicular to the axis of rotation.

We then substitute the expression (2.12) into the right-hand side of (2.13) to get

$$
\begin{aligned}
& \left(\frac{d \mathbf{u}_{F}}{d t}\right)_{F}=\left(\frac{d}{d t}\left(\mathbf{u}_{R}+\boldsymbol{\Omega} \times \mathbf{r}\right)\right)_{R}+\boldsymbol{\Omega} \times\left(\mathbf{u}_{R}+\boldsymbol{\Omega} \times \mathbf{r}\right) \\
& =\left(\frac{d \mathbf{u}_{R}}{d t}\right)_{R}+\mathbf{\Omega} \times\left(\frac{d \mathbf{r}}{d t}\right)_{R}+\mathbf{\Omega} \times \mathbf{u}_{R}+\boldsymbol{\Omega} \times \boldsymbol{\Omega} \times \mathbf{r} .
\end{aligned}
$$

So the acceleration in the fixed frame is given in terms of that in the rotating frame by

$$
\mathbf{a}_{F}=\mathbf{a}_{R}+2 \Omega \times \mathbf{u}_{R}+\Omega \times(\Omega \times \mathbf{r}) .
$$

The last term in (2.15) can be written in terms of a vector $\mathbf{R}$ drawn perpendicular 
to the axis of rotation (Figure 2.2). Clearly from Figure $2.2, \Omega \times \mathbf{r}=\Omega \times \mathbf{R}$ because

$$
|\boldsymbol{\Omega} \times \mathbf{r}|=|\boldsymbol{\Omega}| \mathbf{r}|\sin \alpha=| \boldsymbol{\Omega}|| \mathbf{R} \mid
$$

Using the vector identity $\mathbf{A} \times(\mathbf{B} \times \mathbf{C})=\mathbf{B}(\mathbf{A C})-\mathbf{C}(\mathbf{A B})$ we can rewrite the last term in (2.15) as

$$
\boldsymbol{\Omega} \times(\boldsymbol{\Omega} \times \mathbf{R})=\boldsymbol{\Omega}(\boldsymbol{\Omega R})-|\boldsymbol{\Omega}|^{2} \mathbf{R}
$$

Since $\boldsymbol{\Omega}$ and $\mathbf{R}$ are perpendicular, $\boldsymbol{\Omega} \cdot \mathbf{R}=0$, and equation (2.15) becomes

$$
\mathbf{a}_{\mathbf{F}}=\mathbf{a}_{R}+2 \boldsymbol{\Omega} \times \mathbf{u}_{R}-|\boldsymbol{\Omega}|^{2} \mathbf{R}
$$

So the inertial acceleration in the fixed frame $\mathbf{a}_{F}$ equals the acceleration measured in the rotating system, plus the Coriolis acceleration $2 \boldsymbol{\Omega} \times \mathbf{u}$ and the centripetal acceleration $-|\boldsymbol{\Omega}|^{2} \mathbf{R}$.

In the momentum equation (2.2) we replace the term $\rho \frac{D \mathbf{u}_{\mathbf{F}}}{D t}$ by $\rho \frac{D \mathbf{u}}{D t}+2 \Omega \times \mathbf{u}-$ $|\Omega|^{2} \mathbf{R}$. The centripetal acceleration $-|\Omega|^{2} \mathbf{R}$ is included in gravity for the geopotential surface of geoid. So in the rotating frame of reference, the momentum equation (2.6) can be written as

$$
\frac{D \mathbf{u}}{D t}=-\frac{1}{\rho} \nabla p-2 \Omega \times \mathbf{u}+\mathbf{g}
$$

where the term $2 \Omega \times \mathbf{u}$ is defined as the Coriolis acceleration. 


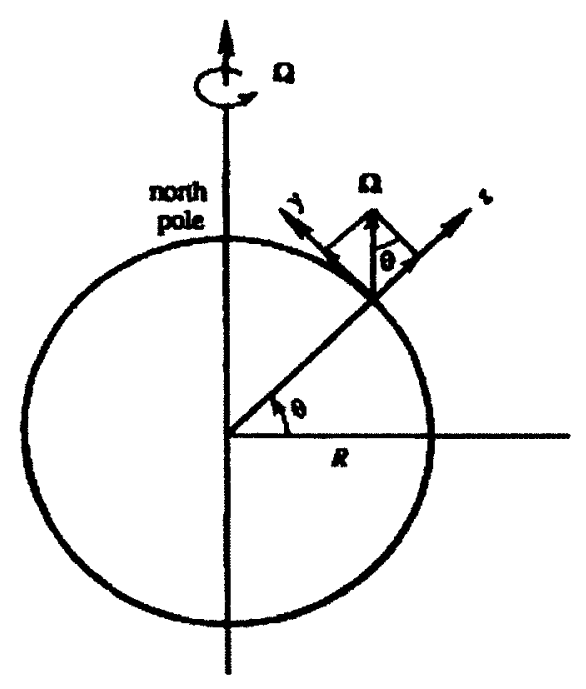

Figure 2.3: Local Cartesian coordinates. The local coordinate system rotates with the Earth. $\Omega$ is the angular velocity of the Earth rotation. $\theta$ is the latitude of the origin of the local coordinate system. $x$ is the west-east horizontal variable, $y$ is the south-north horizontal variable, $z$ is in the vertical direction. This figure is taken from Gill (1982).

\subsection{The Coriolis effect for a thin layer on a rotating sphere}

The atmosphere and the ocean are very thin layers in which the depth scale $H$ of the flow is a few kilometers, but the horizontal scale $L$ is of the order of hundreds or thousands of kilometers. We consider a coordinate frame rotating with the Earth. The Earth rotates with an angular speed $|\Omega|=\Omega=0.73 \cdot 10^{-4} \mathrm{~s}^{-1}$. In the local coordinate system $(x, y, z)$, the angular velocity is $\boldsymbol{\Omega}=(0, \Omega \cos \theta, \Omega \sin \theta)$, where $\theta$ is latitude (see Figure 2.3). The Coriolis force $F$ is

$$
F=2 \rho \Omega \times \mathbf{u}=2 \rho(w \Omega \cos \theta-v \Omega \sin \theta, u \Omega \sin \theta,-u \Omega \cos \theta)
$$


Since the vertical component of the velocity is small compared with the horizontal components $w \ll v, w \ll u$, the Coriolis force can be approximated as

$$
2 \rho \Omega \times \mathbf{u} \approx \rho(-2 v \Omega \sin \theta, 2 u \Omega \sin \theta,-2 u \Omega \cos \theta)
$$

The quantity $f=2 \Omega \sin \theta$ is called the planetary vorticity, or the Coriolis parameter.

\section{Barotropic flow}

A barotropic flow is a fluid flow in which the pressure is a function of the density only, i.e. $p=p(\rho)$. It is a flow in which isobaric constant pressure surfaces are isopycnic surfaces (surfaces of constant density). A two-dimensional flow on a horizontal plane where density variations are neglected is an example of a barotropic flow.

\section{The $f$-plane approximation}

The Coriolis parameter $f=2 \Omega \sin \theta$ varies with latitude $\theta$. The variation is important only for processes having very long time scales or very long length scales. For small scale processes the Coriolis parameter $f$ can considered to be constant: $f=f_{0}=$ $2 \Omega \sin \theta_{0}$ where $\theta_{0}$ is a fixed latitude under consideration. This is called the $f$-plane approximation.

\section{The $\beta$-plane approximation}

A better approximation for the Coriolis parameter $f$ which takes into account the variation of $f$ with latitude can be obtained by linearizing $f$ about some fixed latitude $\theta_{0}$. We consider a plane tangent to the surface of the Earth at the latitude $\theta_{0}$ and define Cartesian coordinates $x$ going from west to east and $y$ going from south to 
north on this plane. We write

$$
f=2 \Omega \sin \theta=2 \Omega \sin \left[\left(\theta-\theta_{0}\right)+\theta_{0}\right]=2 \Omega\left[\sin \left(\theta-\theta_{0}\right) \cos \theta_{0}+\cos \left(\theta-\theta_{0}\right) \sin \theta_{0}\right] .
$$

For latitudes $\theta$ close to $\theta_{0}, \theta-\theta_{0}$ is a small angle, so $\cos \left(\theta-\theta_{0}\right) \approx 1$ and $\sin \left(\theta-\theta_{0}\right) \approx 0$. We use the arclength formula to express the south-north coordinate on the plane in terms of the latitude by $y=R \sin \left(\theta-\theta_{0}\right)$ where $R$ is the radius of the Earth. The Coriolis parameter $f$ can then be approximated as

$$
f=2 \Omega\left(\sin \theta_{0}+\frac{y}{R} \cos \theta_{0}\right)=f_{0}+\beta y
$$

where we define the constants $f_{0}$ and $\beta$ as

$$
\begin{gathered}
f_{0}=2 \Omega \sin \theta_{0}, \\
\beta=\frac{2 \Omega \cos \theta_{0}}{R} .
\end{gathered}
$$

This is called the $\beta$-approximation. It approximates the curved surface of the Earth by a horizontal plane.

\subsection{Waves in the ocean and the atmosphere}

Barotropic vortex Rossby waves are examples of waves that occur in the atmosphere. Mathematically they are represented as sinusoidal perturbations with specified wavelengths and amplitudes. We write a sinusoidal one-dimensional wave in the form

$$
\eta=a \sin \left(\frac{2 \pi}{\lambda}(x-c t)\right)
$$


where the argument $2 \pi(x-c t) / \lambda$ is called the phase of the wave, and $a$ is the amplitude. The parameter $\lambda$ is called the wavelength. The wavenumber is defined as

$$
k=\frac{2 \pi}{\lambda}
$$

which is the number of complete waves in a length of $2 \pi$. The period $T$ of a wave is the time required for the wave to travel one wavelength:

$$
T=\frac{\lambda}{c}
$$

The number of oscillations per unit time at a fixed point in space is the frequency, given by

$$
\nu=\frac{1}{T}
$$

Clearly, $c=\lambda \nu$. The quantity

$$
\omega=2 \pi \nu=k c
$$

is called the circular frequency. The speed of propagation of the wave is given by

$$
c=\frac{\omega}{k}
$$

This is called the phase speed, as it is the rate at which the 'phase' of the wave (i.e. the crests and troughs) propagates. In general the circular frequency is a function depending on the wavenumber $\omega=\omega(k)$. The relation between $\omega$ and $k$ is called a dispersion relation $\omega=\omega(k)$. If the phase speed $\omega / k$ is not constant then the wave is called dispersive. If we consider a wavepacket, which comprises a spectrum of waves of different frequencies and wavenumbers, dispersive waves from a wavepacket propagate with different speed depending on the frequency, and the observed pulse 
changes shape. If $\omega / k$ is constant, the wave is nondispersive, and all waves in a wave packet propagate with the same speed. For the wavepacket we define the group velocity as

$$
c_{g}=\frac{\partial \omega}{\partial k}
$$

For non-dispersive waves the group velocity $c_{g}$ is equal to the phase velocity $c$. For dispersive waves $c_{g} \neq c$. 


\section{Chapter 3}

\section{Vortex dynamics in the atmosphere}

\subsection{Barotropic vorticity equation}

The goal of this thesis is to investigate the dynamics of hurricanes and vortex waves using a mathematical framework based on the equations of motion for a barotropic fluid flow on a horizontal plane tangent to the surface of the Earth. The model comprises a vortex with Rossby waves which are generated near the centre and propagate outwards. It includes time dependence, the effect of the Coriolis force and nonlinear interactions between the vortex and the waves. However, it is simple enough to allow us to derive approximate analytical solutions which give us some insight into possible mechanisms that can lead to the secondary eyewall formation and replacement cycle.

In this chapter we derive the governing equations for our model, i.e. the equations for barotropic vortex Rossby waves, in both rectangular and polar coordinates. We also discuss previous work on the subject of vortex Rossby waves and hurricane dynamics.

Analyses of tropical dynamics (Holton, 1992, section 11.2) show that in the absence of condensation heating, the synoptic-scale dynamics of the tropical atmosphere is 
approximately barotropic when the vertical scale is comparable with the scale height of the atmosphere. So in this thesis we shall describe the hurricane dynamics by the equations of motion for a barotropic fluid flow. We derive the equations in terms of Cartesian coordinates, $x$ and $y$ in a horizontal plane tangent to the surface of the Earth, and $z$ in the vertical direction. The corresponding components of the velocity $\mathbf{u}$ are $u, v$, and $w$.

We derive the potential vorticity equation following Holton (1992) and Kundu and Cohen (2004). Large-scale atmospheric flow can be considered as barotropic and incompressible (see, e.g., Holton, 1992, section 4.5). For such a flow the continuity equation (2.1) simplifies to

$$
\frac{\partial u}{\partial x}+\frac{\partial v}{\partial y}+\frac{\partial w}{\partial z}=0
$$

We consider a shallow layer of fluid of $\operatorname{depth} h$, where $h$ is a function of $x, y$, and $t$. Let $\eta(x, y, t)$ be the vertical displacement of a fluid particle in the flow, and $h(x, y, t)=$ $\eta_{2}(x, y, t)-\eta_{1}(x, y, t)$, where $\eta_{1}$ is the vertical displacement of the liquid particle at the bottom of the layer, and $\eta_{2}$ is the vertical displacement at its surface. In the shallow wave approximation it is assumed that if there are waves in the flow, their wavelength $\lambda$ is much larger than $h$, the depth of the layer $(h / \lambda \ll 1)$. In this case the vertical velocities are much smaller than the horizontal velocities. Then the acceleration $\partial w / \partial t$ is negligible in the vertical component of the momentum equation (2.6). The pressure distribution is assumed to be hydrostatic,

$$
p=p_{0}+\rho g \eta
$$

where $p_{0}$ is the pressure at some reference height. The horizontal pressure gradients 
are therefore

$$
\begin{aligned}
& \frac{1}{\rho} \frac{\partial p}{\partial x}=g \frac{\partial \eta}{\partial x} \\
& \frac{1}{\rho} \frac{\partial p}{\partial y}=g \frac{\partial \eta}{\partial y}
\end{aligned}
$$

Holton showed (Holton, 1992, section 11.2) that in the tropical troposphere a typical scale of the vertical velocity is $0.003 \mathrm{~ms}^{-1}$. For the hurricane dynamics a typical horizontal velocity scale $U$ is $10 \mathrm{~ms}^{-1}$. The vertical length scale $H$ is $10^{4} \mathrm{~m}$, and the horizontal length scale $L$ is $10^{5} \mathrm{~m}$. So the scale of the term $\frac{\partial w}{\partial z}$ in the continuity equation is $W / H$ which is $3 \times 10^{-7} \mathrm{~s}^{-1}$. Scale of the horizontal derivatives $\frac{\partial u}{\partial x}$ and $\frac{\partial v}{\partial y}$ is $U / L$ which is $10^{-4} \mathrm{~s}^{-1}$.

So we see that $W / H \ll U / L$ and can consider $\frac{\partial w}{\partial z}$ to be close to zero, and treat the vertical velocity as being independent of $z$. Hence, from the continuity equation (3.1) the $\operatorname{sum} \frac{\partial u}{\partial x}+\frac{\partial v}{\partial y}$ is independent on $z$ as well. Integrating (3.1) with respect to $z$ across the layer of fluid from $z=\eta_{1}$ at the bottom to $z=\eta_{2}$ at the surface, we obtain

$$
h \frac{\partial u}{\partial x}+h \frac{\partial v}{\partial y}+w\left(\eta_{2}\right)-w\left(\eta_{1}\right)=0
$$

where $w\left(\eta_{2}\right)$ is the vertical velocity at the surface and $w\left(\eta_{1}\right)$ is the vertical velocity at the bottom of the layer. The difference in velocity between the surface and at the bottom is given by

$$
w\left(\eta_{2}\right)-w\left(\eta_{1}\right)=\frac{D\left(\eta_{2}(x, y, t)-\eta_{1}(x, y, t)\right)}{D t}=\frac{D h(x, y, t)}{D t}=\frac{\partial h}{\partial t}+u \frac{\partial h}{\partial x}+v \frac{\partial h}{\partial y}
$$


and the sum of equations (3.4) and (3.5) can be rewritten as

$$
\frac{\partial h}{\partial t}+\frac{\partial}{\partial x}[u h]+\frac{\partial}{\partial y}[v h]=0
$$

Writing the pressure in terms of $\eta$ (3.4) for a fluid shallow flow in a rotating frame, we obtain the horizontal components of the momentum equation (2.19) in the form

$$
\begin{aligned}
& \frac{\partial u}{\partial t}+u \frac{\partial u}{\partial x}+v \frac{\partial u}{\partial y}-f v=-g \frac{\partial \eta}{\partial x} \\
& \frac{\partial v}{\partial t}+u \frac{\partial v}{\partial x}+v \frac{\partial v}{\partial y}+f u=-g \frac{\partial \eta}{\partial y}
\end{aligned}
$$

A vorticity equation can be derived by differentiating (3.7) with respect to $y,(3.8)$ with respect to $x$, and subtracting. We denote the vertical component of the relative vorticity $\nabla \times \mathbf{u}$ by

$$
\zeta=\frac{\partial v}{\partial x}-\frac{\partial u}{\partial y}
$$

In the $\beta$-plane approximation $f$ depends on $y$ only, $f=f_{0}+\beta y$. Then from (3.7), (3.8) and (3.9) we obtain

$$
\frac{D \zeta}{D t}+(\zeta+f)\left(\frac{\partial u}{\partial x}+\frac{\partial v}{\partial y}\right)+v \frac{\partial f}{\partial y}=0
$$

We can write equation (3.6) as

$$
\frac{D h}{D t}+h\left(\frac{\partial u}{\partial x}+\frac{\partial v}{\partial y}\right)=0
$$

and then use it to eliminate the horizontal divergence $\partial u / \partial x+\partial v / \partial y$ from (3.10) to 
obtain

$$
\frac{D \zeta}{D t}=\frac{(\zeta+f)}{h} \frac{D h}{D t}-v \frac{\partial f}{\partial y}
$$

We make use of

$$
\frac{D f}{D t}=v \frac{\partial f}{\partial y}
$$

to write (3.12) as

$$
\frac{D(\zeta+f)}{D t}=\frac{(\zeta+f)}{h} \frac{D h}{D t}
$$

This equation can be written in the compact form

$$
\frac{D}{D t}\left(\frac{\zeta+f}{h}\right)=0
$$

This is the potential vorticity conservation law in the shallow-water approximation.

If the flow is horizontal or at least approximately horizontal then we can consider $w=0$ and from (3.4) obtain that $\partial u / \partial x+\partial v / \partial y=0$. In this case (3.10) is simplified to

$$
\frac{D \zeta}{D t}+v \frac{\partial f}{\partial y}=0
$$

and the barotropic potential vorticity equation is

$$
\frac{D}{D t}(\zeta+f)=0
$$

This equation could also be derived by using the baroclinic potential vorticity equation (Holton, 1992, section 4.6):

$$
\frac{\tilde{D}}{D t}\left(\zeta_{\theta}+f\right)+\left(\zeta_{\theta}+f\right) \nabla_{\theta} \cdot \mathbf{u}=-\mathbf{k} \cdot \nabla_{\theta} \times\left(\frac{D \theta}{D t} \frac{\partial \mathbf{u}}{\partial \theta}\right)
$$

where $\mathbf{k}$ is the unit vector in $z$-direction, $\theta$ is the potential temperature, and the 
subscript $\theta$ means that the derivatives are taken on a surface with constant $\theta$. The potential temperature is by definition the temperature that a parcel of dry air at pressure $p$ and temperature $T$ would acquire if it were expanded or compressed adiabatically to the reference pressure $p_{s}=1000 \mathrm{mb}$ :

$$
\theta=T\left(\frac{p_{s}}{p}\right)^{\kappa}
$$

where $\kappa=R / c_{p}, R$ is the gas constant for the dry air, and $c_{p}$ is the specific heat at constant pressure. The notation $\frac{\bar{D}}{D t}$

$$
\frac{\tilde{D}}{D t}=\frac{\partial}{\partial t}+\mathbf{u} \cdot \nabla_{\theta}
$$

is the total derivative on a surface where $\theta=$ constant.

If we make the assumption that the flow is barotropic, equation (3.18) simplifies to the equation (3.15) which we derived using the shallow water approximation. To show this, we note that

$$
\frac{\tilde{D}}{D t}\left(\zeta_{\theta}+f\right)+\left(\zeta_{\theta}+f\right) \nabla_{\theta} \cdot \mathbf{u}=\frac{D}{D t}\left(\zeta_{\theta}+f\right)
$$

We consider a tropical cyclone to be a two-dimensional vortex in a horizontal plane. For an ideal gas, $\theta$ is constant on a barotropic surface. For this case the operator $\nabla_{\theta}$ is simplified to the horizontal component of the operator $\nabla$, and the vertical vorticity $\zeta_{\theta}=\zeta$. We consider adiabatic flow, so $D \theta / D t=0$. In this case, equation (3.18) reduces to equation $(3.15)$

$$
\frac{D}{D t}(\zeta+f)=0
$$

We will use this equation to obtain an equation for the propagation of barotropic 
Rossby waves.

We will consider a tropical cyclone as a vortex where the vertical scale $H$ is smaller than the horizontal scale $L, H / L \ll 1$. As discussed above in this section, tropical dynamics can be considered to be a barotropic flow and we can neglect the vertical variation of the flow and consider the problem as two-dimensional. So the continuity equation can be approximated by

$$
u_{x}+v_{y}=0
$$

where the subscripts denote partial differentiation. This allows us to define a streamfunction $\Psi(x, y, t)$ by

$$
u=-\Psi_{y}, v=\Psi_{x}
$$

The vorticity can then be expressed in terms of the streamfunction as

$$
\zeta=v_{x}-u_{y}=\nabla^{2} \Psi
$$

We can rewrite equation (3.22) in terms of the streamfunction as

$$
\frac{D\left(\nabla^{2} \Psi+f\right)}{D t}=0
$$

On a $\beta$-plane the Coriolis parameter $f=f_{0}+\beta y$, so we can write

$$
\frac{D \nabla^{2} \Psi}{D t}+\beta v=0
$$

or in the form

$$
\nabla^{2} \Psi_{t}-\Psi_{y} \nabla^{2} \Psi_{x}+\Psi_{x}\left(\nabla^{2} \Psi_{y}\right)=-\beta \Psi_{x}
$$


This equation is called the barotropic vorticity equation. We will use it to describe barotropic Rossby waves.

To simplify further consideration of the problem let us consider the nondimensional form of the barotropic vorticity equation. Let us denote all dimensional variables by an asterisk $*$ and write the dimensional barotropic vorticity equation as:

$$
\nabla^{* 2}\left(\frac{\partial \Psi^{*}}{\partial t^{*}}\right)-\frac{\partial \Psi^{*}}{\partial y^{*}} \nabla^{* 2}\left(\frac{\partial \Psi^{*}}{\partial x^{*}}\right)+\frac{\partial \Psi^{*}}{\partial x^{*}} \nabla^{* 2}\left(\frac{\partial \Psi^{*}}{\partial y^{*}}\right)=-\beta^{*} \frac{\partial \Psi^{*}}{\partial x^{*}}
$$

Suppose the characteristic length scale of our problem is $L$ and the characteristic velocity is $U$. Then the characteristic time scale is $T=L / U$ and the characteristic streamfunction is $\Psi=U L$. We define the nondimensional variables in terms of the corresponding dimensional ones as follows:

$$
x=\frac{x^{*}}{L}, y=\frac{y^{*}}{L}, t=\frac{t^{*}}{T}=\frac{U t^{*}}{L}, \Psi=\frac{\Psi^{*}}{U L}, f=f^{*} \frac{U}{L}, \beta=\beta^{*} \frac{L^{2}}{U} .
$$

This means that the derivatives in terms of the non-dimensional variables will be:

$$
\begin{aligned}
& \frac{\partial}{\partial x^{*}}=\frac{1}{L} \frac{\partial}{\partial x}, \\
& \frac{\partial}{\partial y^{*}}=\frac{1}{L} \frac{\partial}{\partial y}, \\
& \frac{\partial}{\partial t^{*}}=\frac{1}{T} \frac{\partial}{\partial t} .
\end{aligned}
$$

We write equation (3.29) in terms of the non-dimensional variables as

$$
\frac{U^{2}}{L^{2}} \nabla^{2}\left(\frac{\partial \Psi}{\partial t}\right)-\frac{U^{2}}{L^{2}} \frac{\partial \Psi}{\partial y} \nabla^{2}\left(\frac{\partial \Psi}{\partial x}\right)+\frac{U^{2}}{L^{2}} \frac{\partial \Psi}{\partial x} \nabla^{2}\left(\frac{\partial \Psi}{\partial y}\right)=-\frac{U^{2}}{L^{2}} \beta \frac{\partial \Psi}{\partial x}
$$

After simplification, we obtain the nondimensional barotropic vorticity equation in 
the form

$$
\nabla^{2} \Psi_{t}-\Psi_{y} \nabla^{2} \Psi_{x}+\Psi_{x}\left(\nabla^{2} \Psi_{y}\right)=-\beta \Psi_{x}
$$

The parameter $\beta$ in (3.35) is the nondimensional gradient of the Coriolis parameter. To determine the magnitude of $\beta$ we note that the dimensional $\beta^{*}$, according to (2.24), is

$$
\beta^{*}=\frac{2 \Omega_{\text {Earth }}}{R_{\text {Earth }}} \cos \theta_{0}
$$

where $\theta_{0}$ is the latitude of the centre of the vortex. Since the vortex is located in the tropics, $\theta_{0}$ is close to zero. So, with $R_{\text {Earth }} \approx 6.3 \times 10^{6} \mathrm{~km}$ and the angular velocity of the Earth's rotation $\Omega_{E a r t h} \approx 7 \times 10^{-5} \mathrm{~s}^{-1}$, the value of $\beta^{*}$ is close to $2.2 \times 10^{-11}$ $\mathrm{m}^{-1} \mathrm{~s}^{-1}$. The dimension of $\beta^{*}$ is $\mathrm{m}^{-1} \mathrm{~s}^{-1}$. So, the characteristic value of $\beta$ is $B=U / L^{2}$ where $U$ is the characteristic velocity of a hurricane, and $L$ is its radius.

A typical radius of the vortex $L$ is about $2-3 \times 10^{5} \mathrm{~m}$, and a typical speed of the tangential wind $U$ is about $30-100 \mathrm{~ms}^{-1}$. So the nondimensional $\beta$ is

$$
\beta=\frac{\beta^{*}}{U / L^{2}}=\frac{2 \Omega_{E a r t h}}{R_{E a r t h}} \frac{L^{2}}{U} \sim 10^{-2}
$$

We can therefore consider $\beta$ to be a small parameter in our problem, i.e. $\beta \ll 1$.

\subsection{Barotropic Rossby waves}

The barotropic vorticity equation (3.28) can be used to describe the propagation of waves that are known as barotropic Rossby waves. The streamfunction $\Psi$ and vorticity $\nabla^{2} \Psi$ are considered to be the total streamfunction and total vorticity, respectively, each comprising a mean quantity corresponding to the background fluid flow, and a perturbation quantity representing the waves. If the wave amplitude is considered 
small relative to the magnitude of the mean flow, then the equation can be linearized.

If the background flow is taken to be the zonal-mean of the zonal wind and the mean speed assumed to be a constant $U$, then the waves can be represented in the form

$$
\psi=A e^{i(k x+l y-\omega t)}
$$

where $x$ and $y$ are rectangular coordinates, the wavenumbers $k$ and $l$ determine the direction of phase propagation in the $x$ and $y$ directions, $A$ is the wave amplitude, and $\omega$ is the wave frequency. Substituting this form of the wave into the vorticity equation (3.28) and linearizing it we obtain the dispersion relation for barotropic Rossby waves

$$
\omega=U k-\frac{\beta k}{k^{2}+l^{2}}
$$

The group velocity is the gradient of $\omega$ in the wavenumber space

$$
\mathbf{c}_{\mathbf{g}}=\mathbf{i}_{\mathbf{1}} \frac{\partial \omega}{\partial k}+\mathbf{i}_{\mathbf{2}} \frac{\partial \omega}{\partial l}
$$

The phase speed is

$$
\mathbf{c}=\frac{\omega\left(k \mathbf{i}_{1}+l \mathbf{i}_{2}\right)}{k^{2}+l^{2}}
$$

and the phase speed in the $x$-direction can be written in the form

$$
c_{x}=\frac{k \omega}{k^{2}+l^{2}}=\frac{U k^{2}}{k^{2}+l^{2}}-\frac{\beta k^{2}}{\left(k^{2}+l^{2}\right)^{2}}
$$

The negative sign of the $\beta$-term shows that the phase propagation is westward relative to the mean flow. If the eastward current cancels the westward phase speed, giving $c_{x}=0$, stationary waves are formed. 


\subsection{Vortex Rossby waves}

For studying vortex dynamics it is convenient to use polar coordinates, $r$ and $\lambda$, where $r$ is the radial distance from the centre of the vortex, and $\lambda$ is the angle measured from a reference direction, usually to the east. The polar coordinates are defined as $r=\sqrt{x^{2}+y^{2}}, \lambda=\arctan (x / y)$, or $x=r \cos \lambda$ and $y=r \sin \lambda$. The velocity components are $v_{\text {radial }}$ and $v_{\text {azimuthal }}$ in the directions of $r$ and $\lambda$ respectively. In polar coordinates the continuity equation

$$
\nabla \cdot \mathbf{u}=\mathbf{0}
$$

is written as

$$
\frac{1}{r} \frac{\partial}{\partial r}\left(r v_{\text {radial }}\right)+\frac{1}{r} \frac{\partial}{\partial \lambda} v_{\text {azimuthal }}=0
$$

The relations between the streamfunction $\Psi(r, \lambda, t)$ and the velocity components are

$$
\begin{aligned}
& v_{\text {radial }}=-\frac{1}{r} \Psi_{\lambda}, \\
& v_{\text {azimuthal }}=\Psi_{r} .
\end{aligned}
$$

The $x$ and $y$ derivatives of the streamfunction can be written in polar coordinates as

$$
\begin{aligned}
& \Psi_{x}=v=v_{\text {radial }} \cos \lambda+v_{\text {azimuthal }} \sin \lambda=-\frac{1}{r} \Psi_{\lambda} \cos \lambda+\Psi_{r} \sin \lambda \\
& \Psi_{y}=-u=v_{\text {azimuthal }} \sin \lambda-v_{\text {radial }} \cos \lambda=\Psi_{r} \sin \lambda+\frac{1}{r} \Psi_{\lambda} \cos \lambda
\end{aligned}
$$

Substituting (3.46) into (3.28), we can write the barotropic vorticity equation in polar coordinates as

$$
\nabla^{2} \Psi_{t}-\frac{1}{r} \Psi_{\lambda} \nabla^{2} \Psi_{r}+\frac{1}{r} \Psi_{r} \nabla^{2} \Psi_{\lambda}-\frac{\beta}{r} \Psi_{\lambda} \sin \lambda+\beta \Psi_{r} \cos \lambda=0
$$


The basic flow in a cyclonic vortex is generally taken to be a uniform rotation of the vortex depending on the radial variable $r$ and independent of the azimuthal angle $\lambda$ and time $t$, and it can thus be written as $(0, \bar{v}(r))$. The streamfunction $\bar{\psi}(r)$ for the basic flow is given by

$$
\bar{v}(r)=\bar{\psi}^{\prime}(r)
$$

where the prime denotes differentiation with respect to $r$. We also define the angular velocity $\bar{\Omega}(r)$ of the basic flow by $\bar{v}=\bar{\Omega}(r) r$.

The total streamfunction $\Psi(r, \lambda, t)$ and angular and radial velocity components are each decomposed into a contribution from the steady basic flow and a time-dependent perturbation representing the waves as follows,

$$
\Psi(r, \lambda, t)=\bar{\psi}(r)+\varepsilon \psi(r, \lambda, t),
$$

where

$$
v_{\text {azimuthal }}(r, \lambda, t)=\bar{v}(r)+\varepsilon v(r, \lambda, t)
$$

and

$$
v_{\text {radial }}(r, \lambda, t)=\varepsilon u(r, \lambda, t) .
$$

It is assumed that $u, v$ and $\psi$ are $O(1)$, so that the parameter $\varepsilon$ gives a measure of the magnitude of the waves relative to that of the basic flow. Using (3.49) - (3.51), the equation (3.47) can be written in terms of the streamfunction perturbation as

$$
\begin{array}{r}
\left(\frac{\partial}{\partial t}+\frac{\bar{v}}{r} \frac{\partial}{\partial \lambda}\right) \nabla^{2} \psi-\frac{1}{r} \psi_{\lambda} \frac{\partial}{\partial r}\left(\bar{v}_{r}+\frac{1}{r} \bar{v}\right)-\frac{\beta}{r} \psi_{\lambda} \sin \lambda+ \\
+\beta \psi_{r} \cos \lambda \frac{\beta}{\varepsilon} \bar{v} \cos \lambda= \\
-\frac{\varepsilon}{r}\left(\psi_{r} \nabla^{2} \psi_{\lambda}-\psi_{\lambda} \nabla^{2} \psi_{r}\right)
\end{array}
$$

The $\frac{\beta}{\varepsilon}$ term in (3.52) is present because the basic flow velocity $\bar{v}(r)$ does not satisfy 
equation (3.47) with the $\beta$ terms included.

We start our analyses of equation (3.47) by considering the case of waves on an $f$-plane, where the Coriolis parameter $f$ is approximated by $f_{0}$, a nonzero constant. In this configuration we set $\beta=0$ in equation (3.52) and obtain

$$
\left(\frac{\partial}{\partial t}+\frac{\bar{v}}{r} \frac{\partial}{\partial \lambda}\right) \nabla^{2} \psi-\frac{1}{r} \psi_{\lambda} \frac{\partial}{\partial r}\left(\bar{v}_{r}+\frac{1}{r} \bar{v}\right)=-\frac{\varepsilon}{r}\left(\psi_{r} \nabla^{2} \psi_{\lambda}-\psi_{\lambda} \nabla^{2} \psi_{r}\right)
$$

It assumed also that the wave amplitude is small relative to the magnitude of the background flow. So the parameter $\varepsilon \ll 1$. This allows us to linearize equation (3.53) and obtain

$$
\left(\frac{\partial}{\partial t}+\frac{\bar{v}}{r} \frac{\partial}{\partial \lambda}\right) \nabla^{2} \psi-\frac{1}{r} \psi_{\lambda} \frac{\partial}{\partial r}\left(\bar{v}_{r}+\frac{1}{r} \bar{v}\right)=0
$$

We derive approximate analytical solutions to this linear equation first for the case of waves with an amplitude that does not depend on time (chapter 4), and then for the case where the wave amplitude evolves with time (chapter 5 ). In the time-dependent configuration we derive asymptotic solutions that are valid for late time. In the limit of infinite time these time-dependent solutions approach the corresponding steady solutions derived in chapter 4 . Section 6.1 gives a weakly-nonlinear analysis of the nonlinear equation (3.53) in which $\varepsilon$ is considered to be a small parameter but the $\beta$-effect is neglected. This corresponds to the situation where $\beta \ll \varepsilon \ll 1$.

The $\beta$-effect is included in sections 6.2 and 6.3. The goal is to include both nonlinearity and the $\beta$-effect; however as a first step, we investigate the effect of adding $\beta$ terms to the linear problem (section 6.2 ). This corresponds to a situation where $\varepsilon \ll \beta$. In that case the term proportional to $\frac{\beta}{\varepsilon}$ is larger than the $O(1)$ terms in the solution and can not satisfy the specified $O(1)$ boundary conditions. In order to derive a solution in this case we need to neglect this term. We can do so by adding an extra term $\tilde{B}(r, \lambda)=-\frac{\beta}{\varepsilon} \bar{v} \cos (\lambda)$ to (3.47). The main focus of this thesis is on the 
configuration where $\beta \ll \varepsilon$ which is the more likely situation to occur in our problem where $\beta \sim 10^{-2}$ for hurricanes with a length scale $L \sim 10^{5} \mathrm{~m}$. In that case the $O(\beta / \varepsilon)$ term in equation (3.52) gives rise to an extra term in the solution but does not change the qualitative behaviour of the solution. We will discuss the influence of $O(\beta / \varepsilon)$ term on the solution in section 6.3 .

\subsection{Previous theoretical studies of tropical cyclone dynamics}

Several theories have been advanced to describe hurricane dynamics, the physics of outward propagating hurricane bands, secondary eyewall formation and other features of the hurricane structure. Some theories describe the spiral bands as inertia-gravity waves (Abdullah, 1966, Kurihara and Tuleya, 1974, Kurihara, 1976). Some more recent papers describe them as potential-vorticity disturbances (Guinn and Shubert, 1993), while another theory hypothesizes that symmetric instability plays an important role in the spiral band formation (Willoughby at al., 1984).

MacDonald (1968) was the first to propose and qualitatively describe the spiral bands as vortex Rossby waves. Montgomery and Kallenbach (1997) extended the theory of vortex wave propagation by unifying the physics of barotropic vortex axisymmetrization and vortex wave propagation in rapidly rotating vortices. Montgomery and Kallenbach (1997) developed an inviscid model where the waves propagate radially outward from the original eyewall region as spiral bands and participate in wave-mean flow interactions. They integrated the linearized vorticity equation on an $f$-plane for the case of a symmetric vortex with a vorticity profile decreasing monotonically with radius. They derived an exact solution for the wave component corresponding to 
wavenumber one and an approximate solution for the higher wave numbers.

This solution was extended by Schecter and Montgomery (2004). They studied the conditions under which the potential vorticity in the core of a tropical cyclone becomes vertically aligned and horizontally axisymmetric. They showed that the amplitude of vortex Rossby waves decays exponentially outside the core of the cyclone, and the decay rate depends on the gradient of potential vorticity at the critical radius.

Brunet and Montgomery (2002) studied the linear initial-value problem for waves propagating on a smooth circular vortex on an $f$-plane. They developed a theory for a barotropic configuration for a vortex of finite depth with a nonzero Rossby radius of deformation. The Rossby radius of deformation is proportional to $l_{r}=\sqrt{\frac{\Delta \rho}{\rho} D} / f$ where $\rho$ is the density, $D$ is the depth of the flow, and $f$ is the Coriolis parameter. It was shown that the non-dimensional evolution equation for the potential vorticity perturbation depends on one parameter, involving the azimuthal wavenumber, the basic state potential vorticity gradient and the Rossby radius of deformation. The timedependent initial-value problem for this configuration was solved in Hankel transform space.

Brunet and Montgomery (2002) and Schecter and Montgomery (2004) discussed the influence of inertia-buoyancy oscillations, i.e. gravity waves, on the vortex dynamics. They concluded that these oscillations are important for cyclones in the middle latitudes. Schecter and Montgomery (2004) showed that the influence of vortex waves exceeds the effect of gravity wave propagation on the vortex dynamics. Hendricks et al. (2010) included the inertia-gravity waves in their numerical simulations of the vortical motion in a hurricane. They concluded that inertia-gravity waves are insignificant in the process of intensification or decay of the vortex.

Martinez et al. (2010a), Martinez et al. (2010b), Martinez et al. (2011) used the empirical normal mode technique to isolate vortex waves from other datasets. 
They studied the impact of vortex waves on the hurricane dynamics and intensity, concentric eyewall genesis, and other main features of a mature tropical cyclone. The results of the analyses of their simulations led them to conclude that the main features of tropical cyclones can be explained by vortex Rossby waves.

In summary, the conclusions of the above mentioned studies in the hurricane dynamics suggest that vortex Rossby waves play a more significant role than inertiagravity waves. This justifies (at least as a first approximation) the representation of the hurricane dynamics using a barotropic model that includes propagating Rossby waves on a horizontal plane but no inertia-gravity waves or other three-dimensional effects.

The subject of secondary eyewalls and their formation is one of the most important research topics in the dynamics of tropical cyclones. However, there is as yet no unified theory to explain secondary eyewall formation. Kossin and Sitkowski (2009), Sitkowski et al. (2011) developed empirical models based on observational datasets to predict the hurricane dynamics and the appearance of the secondary wind maximum. Willoughby and Shapiro (1982) proposed a model where the eyewall formation is the response of a tropical cyclone to circularly symmetric, convective heat sources. Willoughby et al. (1982) presented numerical simulations which showed that the tangential wind increases rapidly inside the radius of maximum wind and decreases inside the eye near the central axis of the vortex. According to their numerical simulations and aircraft observations, the secondary eyewall often contracts as it intensifies. A secondary eyewall is frequently observed to become narrower and replace a pre-existing eyewall.

Montgomery and Kallenbach (1997) hypothesized that the secondary eyewall formation may take place because of vortex wave propagation in tropical cyclones. The waves transfer angular momentum to the mean flow at the critical radius where their phase velocity matches the mean flow angular velocity. 
Another theory is based on the work of Nong and Emanuel (2003) in which it is proposed that the instability mechanism leading to the secondary eyewall formation results from wind-induced surface heat exchange.

Kuo et al. (2008) and Martinez et al. (2011) used numerical simulations to investigate the mechanisms by which vorticity perturbations around a strong tropical cyclone may form a ring of enhanced vorticity. Martinez et al. (2011) applied a spacetime empirical normal mode statistical technique to study the genesis of a secondary eyewall in a simulated hurricane.

High-resolution, full-physics numerical simulations have also been used to study secondary eyewall formation by Terwey and Montgomery (2008) who hypothesized that the secondary eyewall is the result of an anisotropic upscale energy cascade of convectively generated vorticity anomalies and vortex Rossby wave propagation.

Abarca and Corbosiero (2011) carried out numerical simulations of hurricanes Rita and Katrina and investigated the secondary eyewall formation. The results of their numerical simulations support the idea that vortex Rossby waves play an important role in the process. Secondary eyewall formation in their model occurs at a radius of 65 (80) $\mathrm{km}$ in Katrina (Rita) close to the hypothesized critical radius of the vortex waves. The secondary eyewall formation in their simulations is characterized by maxima in convective activity and potential vorticity maxima in the lower troposphere. Their results support the notion that secondary eyewall formation is intimately related to bands of high precipitable water and potential vorticity variance emanating from the primary eyewall.

The debate about the mechanisms for the secondary eyewall formation is still very active, as seen for example in the recent polemic paper of Terwey et al. (2012) where, based on the numerical simulations in Terwey and Montgomery (2008), the authors argue against some conclusions of Judt and Chen (2010) and present arguments to 
show the importance of vortex Rossby waves in the secondary eyewall formation.

One of the key points to explain the secondary eyewall formation mechanism is the interaction between vortex Rossby waves and the mean flow of the cyclone. Wavemean flow interactions generally take place when waves reach a location in the atmosphere where the background wind speed equals the wave phase speed. This location is called a critical latitude or critical level, or in the case of vortex waves, a critical radius. Montgomery and Kallenbach (1997) developed a balanced numerical model based on vortex wave propagation and their experiments demonstrated that the interaction between the vortex waves and the mean flow at the critical radius may be the primary mechanism for the increase in the angular wind in the vortex. They also suggested that vortex wave dynamics may be a key factor to explain the secondary eyewall formation. Since that work, the concept of the critical layer interaction has been used to explain some features of tropical cyclone dynamics. Brunet and Montgomery (2002) discussed critical layer interaction in their solution and showed its influence on the cyclone dynamics. Martinez et al. (2011) used the empirical normal-mode approach to demonstrate that the maximum cyclonic angular momentum is transported to the location where the secondary eyewall forms. The fact that the critical radius for some modes of vortex waves is contained inside the region where the secondary eyewall forms led researchers to suggest that a wave-mean flow interaction mechanism may be suitable to explain dynamical aspects of concentric eyewall genesis.

In this thesis we will use the concept of the critical layer interaction to investigate the secondary eyewall formation. Interest in critical layer interactions has been stimulated by the suggestion that this mechanism can explain certain phenomena observed in the atmosphere and ocean. For example, Holton and Lindzen (1972) argued that critical layer absorption of gravity waves might be the cause of the changes in mean flow momentum that produce the quasi-biennial oscillation of easterly and westerly 
winds that are observed in the middle atmosphere.

From a mathematical point of view, the critical layer occurs at the point where the steady linear inviscid equation for the wave amplitude has a singularity. The singularity can be avoided by introducing to the critical layer one or more the effects that have been neglected, namely time-dependence, nonlinearity, and viscosity. The introduction of time-dependence was first considered by Booker and Bretherton (1967) in their study of the vertical propagation of gravity waves in a stratified shear flow. They considered a linear, inviscid model and showed that near the critical layer the waves are absorbed and are unable to penetrate above the layer. Analyses by Stewartson (1978) and Warn and Warn $(1976,1978)$ used asymptotic methods to investigate a nonlinear Rossby waves critical layer regime defined by a balance between temporal evolution and nonlinearity. There have since been a number of analytical and numerical studies on the development of Rossby waves critical layers. For example, Campbell and Maslowe (1998) and Campbell (2004) which examined a configuration with a spatially-localized Rossby wave packet.

These investigations all involved Rossby waves in rectangular geometry. A recent study by Caillol (2012) deals with vortex waves in a rapidly rotating vortex. His simplified analytical model is based on the propagation of a vortex Rossby wave in a barotropic and axisymmetric vortex on an $f$-plane. The wave-flow interaction is described in the critical layer where the viscous term is included in the governing equation. This study describes the change of the momentum flux and predicts the existence of multiple vortices in the critical layer.

My thesis investigates the role of vortex Rossby waves in tropical cyclones dynamics and includes:

- a mathematical description for vortex Rossby wave propagation in a cyclonic two-dimensional vortex, 
- solutions for the linear steady problem for waves on an $f$-plane in terms of hypergeometric functions,

- solutions for the linear time-dependent problem for waves on an $f$-plane far away from the critical layer (the outer region) and in the critical layer (the inner region),

- a weakly-nonlinear analysis of the wave-mean flow interaction for waves on an $f$-plane,

- an analysis of the linear equation for waves on a $\beta$-plane, i.e. including the effect of the variation of the Coriolis parameter with latitude,

- an analysis of the combined effects of nonlinearity and the variation of the Coriolis parameter (the $\beta$-effect) on the vortex dynamics,

- an investigation of the change of the mean angular velocity in the critical layer which can explain the formation of the secondary eyewall and its dynamics. 


\section{Chapter 4}

\section{Steady linear problem}

\subsection{Steady linear solution for some special cases}

In this chapter we derive solutions for the linear problem involving vortex waves on an $f$-plane. The governing equation for this configuration is (3.54):

$$
\left(\frac{\partial}{\partial t}+\frac{\bar{v}}{r} \frac{\partial}{\partial \lambda}\right) \nabla^{2} \psi-\frac{1}{r} \psi_{\lambda} \frac{\partial}{\partial r}\left(\bar{v}_{r}+\frac{1}{r} \bar{v}\right)=0
$$

which can be written in terms of the angular velocity $\bar{\Omega}(r)=\bar{v}(r) / r$ as

$$
\left(\frac{\partial}{\partial t}+\bar{\Omega} \frac{\partial}{\partial \lambda}\right) \nabla^{2} \psi-\psi_{\lambda}\left(\bar{\Omega}^{\prime \prime}(r)+3 \bar{\Omega}^{\prime}(r) / r\right)=0
$$

We consider waves with the streamfunction $\psi(r, \lambda, t)$ generated at some fixed distance $r=r_{1}$ from the centre of the vortex, corresponding to the primary eyewall, and propagating outwards in the direction of increasing $r$. The domain for the problem is defined in terms of polar coordinates $r$ and $\lambda$ by $r_{1} \leq r<\infty, 0 \leq \lambda<2 \pi$ with $r=0$ corresponding to the centre of the vortex (see Figure 4.1). The waves are generated by a steady oscillatory boundary condition for the perturbed streamfunction of the 
form

$$
\psi\left(r_{1}, \lambda, t\right)=A\left(e^{i(k \lambda-\omega t)}+e^{-i(k \lambda-\omega t)}\right)
$$

where $A$ is a constant, $k$ is the azimuthal wave number, $k>0$, and $\omega$ is the circular frequency of the wave. We require that $\Psi$ is finite as $r \rightarrow \infty$. The total streamfunction $\psi(r, \lambda, t)$ is, as defined in (3.49),

$$
\Psi(r, \lambda, t)=\bar{\psi}(r)+\varepsilon \psi(r, \lambda, t)
$$

where $\varepsilon \ll 1$ and $\psi(r, \lambda, t)$ is $O(1)$. Thus $A$ is taken to be $O(1)$, and we can set $A=1$.

With the boundary condition (4.3) we can derive a normal mode solution to equation $(4.2)$ in the form

$$
\psi(r, \lambda, t)=\phi(r) e^{i(k \lambda-\omega t)}+\mathrm{c.c.}
$$

where c.c. denotes the complex conjugate of the preceding term and $\phi(r)$ is the complex wave amplitude. By adding the complex conjugate we ensure that $\psi$ is real although $\phi$ generally has a nonzero imaginary part. The form (4.5) assumes that the wave amplitude is a function of the radial variable only and does not evolve with time. In chapter 5 we consider the case where the amplitude evolves with time.

After substituting this normal mode form into the linear equation (4.2) we obtain an ordinary differential equation for the wave amplitude $\phi(r)$,

$$
\phi^{\prime \prime}+\frac{\phi^{\prime}}{r}-\frac{k^{2}}{r^{2}} \phi+\frac{k\left(\bar{\Omega}^{\prime \prime}(r)+3 \bar{\Omega}^{\prime} / r\right)}{\omega-k \bar{\Omega}} \phi=0
$$

with boundary conditions $\phi\left(r_{1}\right)=1$ and $\phi(r)$ finite as $r \rightarrow \infty$.

We see that the equation is singular at $r=r_{c}$, where $\bar{\Omega}\left(r_{c}\right)=\frac{\omega}{k}$. This radius is the radius where the angular velocity of the vortex $\bar{\Omega}(r)$ equals the phase speed of the waves and is called the critical radius, and the region surrounding the critical radius 


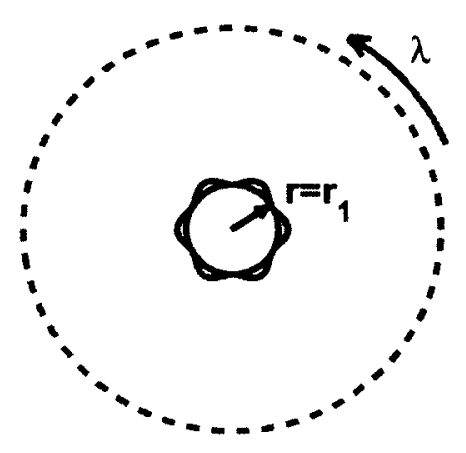

Figure 4.1: The domain of the problem. The domain is $r_{1} \leq r<\infty, 0 \leq \lambda<2 \pi$ with $r=0$ corresponding to the centre of the vortex, $r$ and $\lambda$ are polar coordinates. Waves are generated at the primary eyewall $r=r_{1}$.

is called the critical layer.

We only consider cases where the mean flow angular velocity is an analytic function of $r$. This means that a solution of equation (4.6) in the vicinity of the critical radius could be derived as a series in powers of $\left(r-r_{c}\right)$ using the method of Frobenius. A second solution would include a logarithmic term $\log \left(r-r_{c}\right)$.

We examine some specific profiles of angular velocity $\bar{\Omega}(r)=\bar{v} / r$ which allow an exact solution of (4.6) to be obtained. These profiles are approximations of the observed profile of the angular velocity in a tropical cyclone and have been studied by previous researchers by analytical methods and numerical simulations.

We first consider the case where $\bar{\Omega}(r)$ is constant and there is no critical radius. We then consider the case where $\bar{\Omega}(r)$ is a quadratic function of $r$. This is the profile that will be used throughout the thesis. 


\section{The case where $\bar{\Omega}=\Omega_{0}$, a constant}

If the angular velocity $\bar{\Omega}$ is constant, then the equation for the wave amplitude $\phi(r)$ (4.6) becomes

$$
\phi^{\prime \prime}+\frac{\phi^{\prime}}{r}-\frac{k^{2}}{r^{2}} \phi=0
$$

which is a Cauchy-Euler equation. Its solution is

$$
\phi=C_{1} r^{k}+C_{2} r^{-k}
$$

where $C_{1}$ and $C_{2}$ are constants. In order for $\phi$ to be bounded at large $r, C_{1}=0$. The boundary condition $\phi\left(r_{1}\right)=1$ tells us that $C_{2}=1$, and so the solution is wave with amplitude decreasing with distance from the hurricane center

$$
\phi=r^{-k}
$$

\section{The case where $\bar{\Omega}$ is a quadratic function of $r$}

Martinez et al. (2010a), Martinez et al. (2010b) used an exponential profile of the basic flow angular velocity in their numerical simulations of hurricane dynamics. This profile is based on typical flows observed in tropical cyclones. Brunet and Montgomery (2002) argued that the angular velocity of a cyclone can be approximated by a quadratic function of $r$. Based on that, we will use

$$
\bar{\Omega}(r)=\Omega_{0}\left(1-\alpha r^{2}\right), \quad \alpha>0
$$

as the angular velocity profile. This gives an approximation for the exponential profile $\Omega_{0} e^{-\alpha r^{2}}$ for $r<1 / \sqrt{\alpha}$ (see Figure 4.2). 


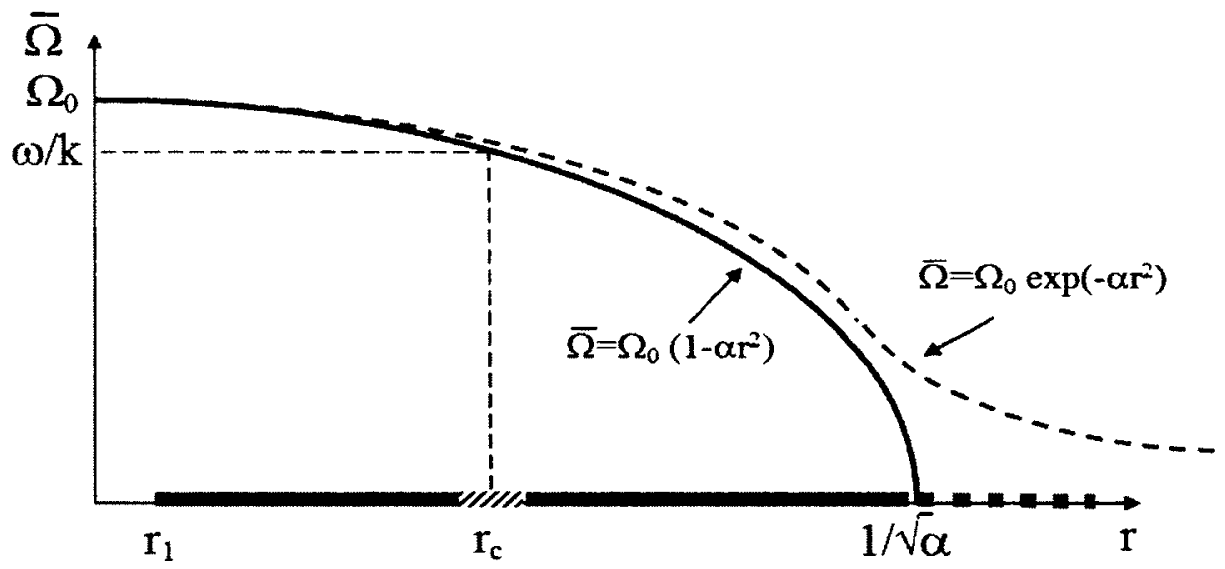

Figure 4.2: The angular velocity profile. The angular velocity $\bar{\Omega}(r)=\Omega_{0}\left(1-\alpha r^{2}\right)$ used in our investigation is an approximation for the exponential profile $\bar{\Omega}(r)=$ $\Omega_{0} e^{-\alpha r^{2}}, r_{1}$ is the location of the primary eyewall, $r_{c}$ is the critical radius and corresponds to the point where $\bar{\Omega}\left(r_{c}\right)=\omega / k$.

With the angular velocity profile (4.10) the equation (4.6) becomes

$$
\phi^{\prime \prime}+\frac{\phi^{\prime}}{r}-\frac{k^{2}}{r^{2}} \phi-\frac{8 k \alpha \Omega_{0}}{\omega-\Omega_{0} k\left(1-\alpha r^{2}\right)} \phi=0
$$

This equation has regular singular points at the center of the vortex where $r=0$ and at $r \rightarrow \infty$. The regular singular point at $r=0$ is not in the domain of our problem and does not need to be considered. There may also be a regular singular point at the critical radius,

$$
r_{c}=\left(\frac{\Omega_{0} k-\omega}{\Omega_{0} \alpha k}\right)^{1 / 2}
$$

where the denominator of the last term in (4.11) becomes zero. The critical radius is located where the angular velocity of the vortex equals the phase speed of the waves

$$
\omega=\Omega_{0} k\left(1-\alpha r_{c}^{2}\right)
$$


The form of the solution of (4.11) depends on the choice of $\omega, \Omega_{0}$, and $\mathrm{k}$. There are three possibilities: $\omega=\Omega_{0} k, \omega>\Omega_{0} k$, and $\omega<\Omega_{0} k$.

\section{The cases where $\omega=\Omega_{0} k$ and $\omega>\Omega_{0} k$}

If $\omega=\Omega_{0} k$, then equation (4.11) becomes

$$
\phi^{\prime \prime}+\frac{\phi^{\prime}}{r}-\frac{k^{2}+8}{r^{2}} \phi=0,0<r_{1}<r<\infty
$$

which is an Euler equation. There are no singular points in the domain of the problem. The general solution is

$$
\phi(r)=C_{1} r^{\sqrt{k^{2}+8}}+C_{2} r^{-\sqrt{k^{2}+8}}
$$

In order for the solution to be bounded for large $r, C_{1}=0$ and the solution satisfying the boundary condition $\phi\left(r_{1}\right)=1$ is

$$
\phi=\left(r / r_{1}\right)^{-\sqrt{k^{2}+8}}
$$

If $\omega>\Omega_{0} k$, then again there are no singular points in the domain of the problem. If $r_{1}$ is small then we can examine the behaviour of the solution for small $r$. In that case we can write equation (4.11) as

$$
\phi^{\prime \prime}+\frac{\phi^{\prime}}{r}-\left(k^{2}+\frac{8 k \alpha \Omega_{0}}{\omega-\Omega_{0} k} r^{2}\right) \phi+O\left(r^{2}\right)=0
$$

So at leading-order, the solution can be expressed in terms of modified Bessel functions of the first and second kind

$$
\phi(r)=C_{1} I_{k}\left(\sqrt{\frac{8 k \alpha \Omega_{0}}{\omega-\Omega_{0} k}} r\right)+C_{2} K_{k}\left(\sqrt{\frac{8 k \alpha \Omega_{0}}{\omega-\Omega_{0} k}} r\right), r_{1}<r \ll 1
$$




\section{The case where $\omega<\Omega_{0} k$}

The most interesting case for our problem is that in which $\omega<\Omega_{0} k$. This is the focus of our investigation for the rest of the thesis. In this case equation (4.11) has a regular singular point at

$$
r_{c}=\left(\frac{k \Omega_{0}-\omega}{k \alpha \Omega_{0}}\right)^{1 / 2}
$$

We will show that equation (4.11) can be transformed into a hypergeometric equation. In order to do so we rewrite it as

$$
\left(\omega-\Omega_{0} k+\Omega_{0} k \alpha r^{2}\right)\left(\phi^{\prime \prime}+\frac{\phi^{\prime}}{r}-\frac{k^{2}}{r^{2}} \phi\right)-8 k \alpha \Omega_{0} \phi=0
$$

or

$$
\left(-\frac{k \Omega_{0}-\omega}{k \alpha \Omega_{0}}+r^{2}\right)\left(\phi^{\prime \prime}+\frac{\phi^{\prime}}{r}-\frac{k^{2}}{r^{2}} \phi\right)-8 \phi=0
$$

and then make the change of variables

$$
\hat{r}=r / r_{c}=\sqrt{\frac{k \alpha \Omega_{0}}{k \Omega_{0}-\omega}} r
$$

where $r_{c}$ is the critical radius given by (4.19). We also define a new variable $\hat{\phi}$ by $\hat{\phi}(\hat{r})=\phi(r)$.

The derivatives of $\phi$ in terms of the new variables are

$$
\phi^{\prime}(r)=\frac{1}{r_{c}} \hat{\phi}^{\prime}(\hat{r}), \quad \phi^{\prime \prime}(r)=\frac{1}{r_{c}^{2}} \hat{\phi}^{\prime \prime}(\hat{r})
$$

In terms of $\hat{\phi}$ and $\hat{r}$, equation (4.21) can be written as

$$
\left(-1+\hat{r}^{2}\right)\left(\hat{\phi}^{\prime \prime}+\frac{\hat{\phi}^{\prime}}{\hat{r}}-\frac{k^{2}}{\hat{r}^{2}} \hat{\phi}\right)-8 \hat{\phi}=0
$$


or

$$
\hat{\phi}^{\prime \prime}+\frac{\hat{\phi}^{\prime}}{\hat{r}}-\frac{k^{2}}{\hat{r}^{2}} \hat{\phi}-\frac{8}{\hat{r}^{2}-1} \hat{\phi}=0
$$

This equation has regular singular points at $\hat{r}=1$ where the denominator in the last term of equation (4.11) is zero, corresponding to the critical radius $r=r_{c}$.

Following Erdelyi (1953), we seek a solution for (4.25) in the form

$$
\hat{\phi}(\hat{r})=\hat{r}^{p} f\left(\hat{r}^{2}\right)
$$

in order to reduce equation (4.20) to a hypergeometric equation. The first and the second derivatives of $\hat{\phi}(\hat{r})$ with respect to $\hat{r}$ are

$$
\hat{\phi}^{\prime}(\hat{r})=p \hat{r}^{p-1} f\left(\hat{r}^{2}\right)+2 \hat{r}^{p+1} f^{\prime}\left(\hat{r}^{2}\right)
$$

and

$$
\hat{\phi}^{\prime \prime}(\hat{r})=p(p-1) \hat{r}^{p-2} f\left(\hat{r}^{2}\right)+2(2 p+1) \hat{r}^{p} f^{\prime}\left(\hat{r}^{2}\right)+4 \hat{r}^{p+2} f^{\prime \prime}\left(\hat{r}^{2}\right)
$$

where the primes on the right-hand side represent derivatives with respect to $\hat{r}^{2}$. Substituting (4.26)-(4.28) into (4.24) we obtain

$$
\begin{aligned}
& \hat{r}^{2}\left(\hat{r}^{2}-1\right)\left[p(p-1) \hat{r}^{p-2} f\left(\hat{r}^{2}\right)+2(2 p+1) \hat{r}^{p} f^{\prime}\left(\hat{r}^{2}\right)+4 \hat{r}^{p+2} f^{\prime \prime}\left(\hat{r}^{2}\right)\right] \\
& \quad+\hat{r}^{2}\left(\hat{r}^{2}-1\right)\left[p \hat{r}^{p-2} f\left(\hat{r}^{2}\right)+2 \hat{r}^{p} f^{\prime}\left(\hat{r}^{2}\right)\right]-k^{2} \hat{r}^{p}\left(\hat{r}^{2}-1\right) f\left(\hat{r}^{2}\right)-8 \hat{r}^{p+2} f\left(\hat{r}^{2}\right)=0
\end{aligned}
$$

After some simplifications we get

$$
\begin{aligned}
4 \hat{r}^{4}\left(\hat{r}^{2}-1\right) f^{\prime \prime}\left(\hat{r}^{2}\right)+4 \hat{r}^{2}\left(\hat{r}^{2}-1\right)(p+1) f^{\prime}\left(\hat{r}^{2}\right)+ & \hat{r}^{2}\left[p^{2}-k^{2}-8\right] f\left(\hat{r}^{2}\right) \\
& +\left[k^{2}-p^{2}\right] f\left(\hat{r}^{2}\right)=0
\end{aligned}
$$


This equation can be transformed into a hypergeometric equation if the last term is set to be zero. This means

$$
p^{2}=k^{2}
$$

and so $p$ is either $k$ or $-k$.

We also introduce the new variable $z=\hat{r}^{2}$, so the equation for $f$ can be written as

$$
z(z-1) f^{\prime \prime}(z)+(z-1)(p+1) f^{\prime}(z)-2 f(z)=0
$$

where the primes denote differentiation with respect to $z$. This is a hypergeometric equation. A hypergeometric equation is generally written in the form (see Abramowitz and Stegun, 1964)

$$
z(1-z) f^{\prime \prime}+(c-(a+b+1) z) f^{\prime}-(a b) f=0 .
$$

Writing equation (4.32) in the form (4.33)

$$
z(1-z) f^{\prime \prime}+((p+1)-(p+1) z) f^{\prime}-(-2) f=0
$$

we see that

$$
\begin{gathered}
a+b+1=p+1 \text { and } a b=-2 . \\
\text { If } p=k, a=\frac{k+\sqrt{k^{2}+8}}{2}, b=\frac{k-\sqrt{k^{2}+8}}{2}, \text { and } c=k+1 . \\
\text { If } p=-k, a=\frac{-k+\sqrt{k^{2}+8}}{2}, b=\frac{-k-\sqrt{k^{2}+8}}{2}, \text { and } c=-k+1 .
\end{gathered}
$$

Equation (4.34) has three singular points, at $z=0, z=1$ and $z \rightarrow \infty$. As already noted, $z=0$ corresponds to $r=0$ and it is not in the domain of the problem. The solution of the hypergeometric equation close to point $z=1$ can be written in the 
form of hypergeometric functions (see the detailed description of the hypergeometric equation and its solutions in Appendix A). Two linearly independent solutions of the hypergeometric equation (4.34) for $z$ close to $1,0<|z-1|<1$, are

$$
f_{1(1)}(z)=(1-z) F(a+1, b+1 ; 2 ;(1-z))
$$

and

$$
\begin{array}{r}
f_{2(1)}(z)=(1-z) F(a+1, b+1 ; 2 ;(1-z)) \log (1-z)+ \\
(1-z) \sum_{n=1}^{\infty} \frac{(a+1)_{(n)}(b+1)_{(n)}}{(2)_{(n)} n !}(1-z)^{n} \\
(\chi(a+1+n)-\chi(a+1)+\chi(b+1+n)-\chi(b+1)- \\
\chi(2+n)+\chi(2)-\chi(n+1)+\chi(1))+\frac{1}{a b} .
\end{array}
$$

where $(a)_{n}=\Pi_{i=0}^{n-1}(a+i)$ is the Pochhammer symbol, and $F$ is the hypergeometric function (see Appendix A) given by

$$
F(a, b, c, z)=\sum_{n=0}^{\infty} \frac{a_{(n)} b_{(n)}}{c_{(n)} n !} z^{n}
$$

The function $\chi(z)$ is

$$
\chi(z)=\frac{d \log (\Gamma(z))}{d z}
$$

The solutions $f_{1(1)}(z)$ and $f_{2(1)}(z)$ are valid for $0<|z-1|<1$. Following Abramowitz and Stegun (1964) we use the subscripts 1(1) and 2(1) to indicate that the solutions (4.38) and (4.39) are valid close to the singular point $z=1$. So with the constants $a$, $b, c$ given by (4.37) and (4.36), the two linearly independent solutions of (4.32) can 
be written in terms of $r^{2} / r_{c}^{2}$ as

$$
\begin{array}{r}
f_{1(1)}\left(\frac{r^{2}}{r_{c}^{2}}\right)=\left(1-\frac{r^{2}}{r_{c}^{2}}\right) \sum_{n=0}^{\infty} \frac{\left(\frac{ \pm k+\sqrt{k^{2}+8}}{2}+1\right)_{(n)}\left(\frac{ \pm k-\sqrt{k^{2}+8}}{2}+1\right)_{(n)}}{(2)_{(n)} n !}\left(1-\frac{r^{2}}{r_{c}^{2}}\right)^{n} \\
0<\left|1-\frac{r^{2}}{r_{c}^{2}}\right|<1
\end{array}
$$

and

$$
\begin{aligned}
& f_{2(1)}\left(\frac{r^{2}}{r_{c}^{2}}\right)=-\frac{1}{2} \\
& +\left(1-\frac{r^{2}}{r_{c}^{2}}\right) \sum_{n=0}^{\infty} \frac{\left(\frac{ \pm k+\sqrt{k^{2}+8}}{2}+1\right)_{(n)}\left(\frac{ \pm k-\sqrt{k^{2}+8}}{2}+1\right)_{(n)}}{(2)_{(n)} n !}\left(1-\frac{r_{2}}{r_{c}^{2}}\right)^{n} \log \left(1-\frac{r^{2}}{r_{c}^{2}}\right) \\
& +\left(1-\frac{r^{2}}{r_{c}^{2}}\right) \sum_{n=1}^{\infty} \frac{\left(\frac{ \pm k+\sqrt{k^{2}+8}}{2}+1\right)_{(n)}\left(\frac{ \pm k-\sqrt{k^{2}+8}}{2}+1\right)_{(n)}}{(2)_{(n)} n !}\left(1-\frac{r_{2}}{r_{c}^{2}}\right)^{n} \\
& {\left[\chi\left(\frac{ \pm k+\sqrt{k^{2}+8}}{2}+1+n\right)-\chi\left(\frac{ \pm k+\sqrt{k^{2}+8}}{2}+1\right)\right.} \\
& +\chi\left(\frac{ \pm k-\sqrt{k^{2}+8}}{2}+1+n\right)-\chi\left(\frac{ \pm k-\sqrt{k^{2}+8}}{2}+1\right)-\chi(2+n) \\
& +\chi(2)-\chi(n+1)+\chi(1)] \text {, } \\
& 0<\left|1-\frac{r^{2}}{r_{c}^{2}}\right|<1
\end{aligned}
$$

These solutions are valid in the intervals $r_{1}<r<r_{c}$ and $r_{c}<r<\sqrt{2} r_{c}$ (see Figure 4.3).

Recall that we searched for a solution of the form of $\phi(r)=\hat{\phi}(\hat{r})=\hat{r}^{p} f\left(\hat{r}^{2}\right)$ (4.26). Thus, the solution of equation (4.20) close to $r=r_{c}$ is proportional to a linear combination of $f_{1(1)}$ and $f_{2(1)}$

$$
\phi(r)=\left(\frac{r}{r_{c}}\right)^{ \pm k}\left[a_{1(1)} f_{1(1)}\left(\frac{r^{2}}{r_{c}^{2}}\right)+a_{2(1)} f_{2(1)}\left(\frac{r^{2}}{r_{c}^{2}}\right)\right]
$$

where $a_{1(1)}$ and $a_{2(1)}$ are constants. Applying the boundary condition that $\phi\left(r_{1}\right)=1$ 


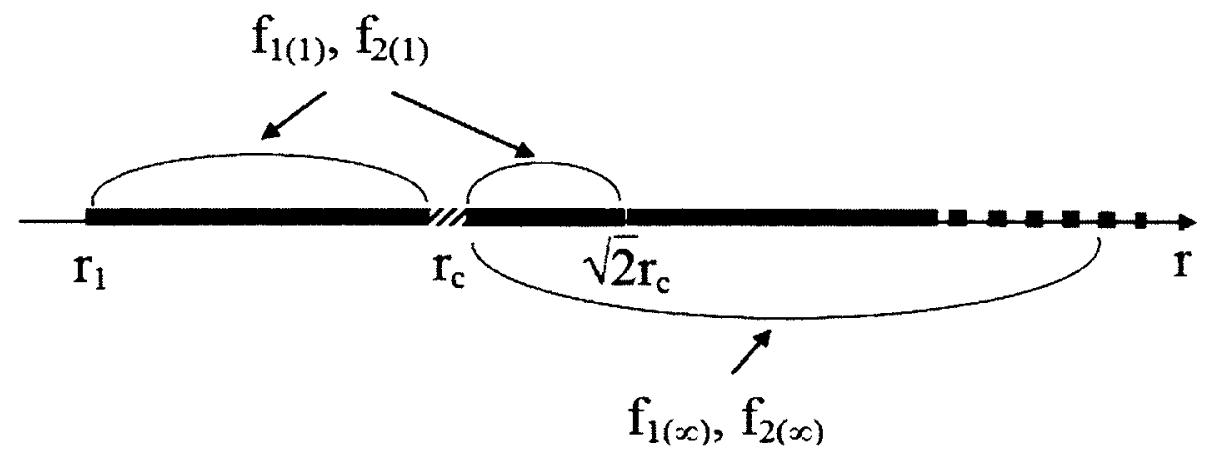

Figure 4.3: Intervals for the steady solution. The solutions $f_{1(1)}\left(\frac{r^{2}}{r_{c}^{2}}\right)$ and $f_{2(1)}\left(\frac{r^{2}}{r_{c}^{2}}\right)$, given by (4.43) and (4.42), are valid for $r_{1}<r<\sqrt{2} r_{c}, r \neq r_{c}$. The solutions $f_{1(\infty)}\left(\frac{r^{2}}{r_{c}^{2}}\right)$ and $f_{2(\infty)}\left(\frac{r^{2}}{r_{c}^{2}}\right)$, given by (4.51) and (4.52), are valid for $r>r_{c}$. In the black interval, $r_{c}<r<\sqrt{2} r_{c}$, the solutions overlap.

we can write the solution in the form

$$
\phi(r)=\left(\frac{r}{r_{1}}\right)^{ \pm k} \frac{f_{1(1)}\left(\frac{r^{2}}{r_{c}^{2}}\right)+a_{1} f_{2(1)}\left(\frac{r^{2}}{r_{c}^{2}}\right)}{f_{1(1)}\left(\frac{r_{1}^{2}}{r_{c}^{2}}\right)+a_{1} f_{2(1)}\left(\frac{r_{1}^{2}}{r_{c}^{2}}\right)}
$$

where $a_{1}=\frac{a_{2(1)}}{a_{1(1)}}$. The solutions $f_{1(1)}$ and $f_{2(1)}$ are valid for $0<\left|1-\frac{r^{2}}{r_{c}^{2}}\right|<1$, i.e. in the intervals $r_{1}<r<r_{c}$ and $r_{c}<r<\sqrt{2} r_{c}$. The leading-order terms of the solution are

$$
\begin{array}{r}
\phi(r) \sim r^{ \pm k}\left[O(1)+O\left(\left(1-\frac{r^{2}}{r_{c}^{2}}\right) \log \left(1-\frac{r^{2}}{r_{c}^{2}}\right)\right)+O\left(1-\frac{r^{2}}{r_{c}^{2}}\right)\right] \\
\sim r^{ \pm k}\left[O(1)+O\left(\left(r_{c}-r\right) \log \left(r_{c}-r\right)\right)+O\left(r_{c}-r\right)\right] \\
0<\left|1-\frac{r^{2}}{r_{c}^{2}}\right|<1 .
\end{array}
$$


or, taking into account that

$$
\log \left(1-\frac{r^{2}}{r_{c}^{2}}\right)=\log \left(r_{c}-r\right)+\log \left(r_{c}+r\right)-\log \left(r_{c}^{2}\right)
$$

we can write the leading-order terms of $(4.44)$ in the form

$$
\begin{array}{r}
\phi(r) \sim r^{ \pm k}\left[O(1)+O\left(\left(r_{c}-r\right) \log \left(r_{c}-r\right)+O\left(r_{c}-r\right)\right]\right. \\
0<\left|1-\frac{r^{2}}{r_{c}^{2}}\right|<1 .
\end{array}
$$

The logarithmic term in $(4.45)$ is $\left(1-\frac{r^{2}}{r_{c}^{2}}\right) \log \left(1-\frac{r^{2}}{r_{c}^{2}}\right)$ where

$$
\log \left(1-\frac{r^{2}}{r_{c}^{2}}\right)=\log \left|1-\frac{r^{2}}{r_{c}^{2}}\right|+i \arg \left(1-\frac{r^{2}}{r_{c}^{2}}\right)
$$

For $r<r_{c}, \arg \left(1-\frac{r^{2}}{r_{c}^{2}}\right)=0$; for $r>r_{c}, \arg \left(1-\frac{r^{2}}{r_{c}^{2}}\right)= \pm \pi$. The sign of the argument is determined by considering the time-dependent problem (see Appendix $\mathrm{C}$ of this thesis). It is found that $\arg \left(1-\frac{r^{2}}{r_{c}^{2}}\right)=-\pi$. So there is a phase shift of $-\pi$ across the critical layer from $r<r_{c}$ to $r>r_{c}$ and the amplitude of the solution is discontinuous across the critical layer. This is analogous to the conclusions of previous researchers for the problem of forced Rossby waves in a rectangular domain (e.g. Warn and Warn, 1978).

For $z>1$ the solution of (4.32) is proportional to a linear combination of the functions defined in (A.17) and (A.18):

$$
\begin{aligned}
& f_{1(\infty)}(z)=z^{-a} F\left(a, a-c+1 ; a-b+1 ; \frac{1}{z}\right), \quad|z|>1 \\
& f_{2(\infty)}(z)=z^{-b} F\left(b, b-c+1 ; b-a+1 ; \frac{1}{z}\right), \quad|z|>1 .
\end{aligned}
$$


In terms of our problem these two solutions are

$$
\begin{aligned}
& f_{1(\infty)}\left(\frac{r^{2}}{r_{c}^{2}}\right)=\left(\frac{r^{2}}{r_{c}^{2}}\right)^{\frac{\mp k-\sqrt{k^{2}+8}}{2}} \sum_{n=0}^{\infty} \frac{\left(\frac{-k+\sqrt{k^{2}+8}}{2}\right)_{n},\left(\frac{k+\sqrt{k^{2}+8}}{2}\right)_{n}}{n !\left(\sqrt{k^{2}+8}+1\right)_{n}}\left(\frac{r^{2}}{r_{c}^{2}}\right)^{-n}, r>r_{c}, \\
& f_{2(\infty)}\left(\frac{r^{2}}{r_{c}^{2}}\right)=\left(\frac{r^{2}}{r_{c}^{2}}\right)^{\frac{ \pm k+\sqrt{k^{2}+8}}{2}} \sum_{n=0}^{\infty} \frac{\left(\frac{-k-\sqrt{k^{2}+8}}{2}\right)_{n},\left(\frac{k-\sqrt{k^{2}+8}}{2}\right)_{n}}{n !\left(-\sqrt{k^{2}+8}+1\right)_{n}}\left(\frac{r^{2}}{r_{c}^{2}}\right)^{-n}, r>r_{c} .
\end{aligned}
$$

The solution of equation (4.20), valid in the interval $\left|\frac{r}{r_{c}}\right|>1$, or $r>r_{c}$ (see Figure 4.3 ), is proportional to a linear combination of $f_{1(\infty)}$ and $f_{2(\infty)}$

$$
\phi(r)=\left(\frac{r}{r_{c}}\right)^{ \pm k}\left[a_{1(\infty)} f_{1(\infty)}\left(\frac{r^{2}}{r_{c}^{2}}\right)+a_{2(\infty)} f_{2(\infty)}\left(\frac{r^{2}}{r_{c}^{2}}\right)\right], r>r_{c}
$$

where $a_{1(\infty)}$ and $a_{2(\infty)}$ are constants. The leading-order terms in the solution are

$$
\begin{aligned}
\left(\frac{r}{r_{c}}\right)^{ \pm k} f_{1(\infty)}\left(\frac{r^{2}}{r_{c}^{2}}\right) & \sim\left(\frac{r}{r_{c}}\right)^{ \pm k}\left(\frac{r^{2}}{r_{c}^{2}}\right)^{\frac{\mp k-\sqrt{k^{2}+8}}{2}}\left[1+O\left(\frac{r}{r_{c}}\right)^{-2}\right] \\
& \sim\left(\frac{r}{r_{c}}\right)^{-\sqrt{k^{2}+8}}\left[1+O\left(\frac{r}{r_{c}}\right)^{-2}\right], r>r_{c}
\end{aligned}
$$

and

$$
\begin{aligned}
\left(\frac{r}{r_{c}}\right)^{ \pm k} f_{2(\infty)}\left(\frac{r^{2}}{r_{c}^{2}}\right) & \sim\left(\frac{r}{r_{c}}\right)^{ \pm k}\left(\frac{r^{2}}{r_{c}^{2}}\right)^{ \pm k+\sqrt{k^{2}+8}} \frac{\left.1+O\left(\frac{r}{r_{c}}\right)^{-2}\right]}{}\left[\left(\frac{r}{r_{c}}\right)^{ \pm \sqrt{k^{2}+8}}\left[1+O\left(\frac{r}{r_{c}}\right)^{-2}\right], r>r_{c} .\right.
\end{aligned}
$$

The term with the positive power is divergent for $r \rightarrow \infty$. So the coefficient $a_{2(\infty)}=0$ 
and the solution is

$$
\phi(r)=\left(\frac{r}{r_{c}}\right)^{ \pm k} a_{1(\infty)} f_{1(\infty)}\left(\frac{r^{2}}{r_{c}^{2}}\right) \sim O\left(\left(\frac{r}{r_{c}}\right)^{-\sqrt{k^{2}+8}}\right), \quad r>r_{c}
$$

as expected from (4.16), and satisfies the condition $\phi(r) \rightarrow 0$ as $r \rightarrow \infty$.

We need to match the solution (4.56) with the solution (4.48). The solution (4.48) is valid in the intervals $r_{1}<r<r_{c}$ and $r_{c}<r<\sqrt{2} r_{c}$ (see Figure 4.3), while the solution (4.56) is valid for $r>r_{c}$. The two solutions should match in the overlapping interval $r_{c}<r<\sqrt{2} r_{c}$ (the black interval on Figure 4.3). The solution (4.56) decreases in this interval with increasing $r$, so the solution (4.48) should also be decreasing in the interval. To satisfy this condition we have to choose the factor $r^{-k}$ in (4.48).

With the negative sign of $k$, the wave amplitude (4.45) in the interval $r_{1}<r<r_{c}$ and $r_{c}<r<\sqrt{2} r_{c}$ is

$$
\begin{array}{r}
\phi(r) \sim\left(\frac{r^{-k}}{\alpha_{1}}\right)\left[1-2\left(1-\frac{r^{2}}{r_{c}^{2}}\right) \log \left(1-\frac{r^{2}}{r_{c}^{2}}\right)+O\left(1-\frac{r^{2}}{r_{c}^{2}}\right)\right], \\
0<\left|1-\frac{r^{2}}{r_{c}^{2}}\right|<1,
\end{array}
$$

where $\alpha_{1}$ is the denominator of (4.45)

$$
\alpha_{1}=f_{1(1)}\left(\frac{r_{1}^{2}}{r_{c}^{2}}\right)+a_{1} f_{2(1)}\left(\frac{r_{1}^{2}}{r_{c}^{2}}\right)
$$

So the solution of $(4.20)$ is

$$
\phi(r)= \begin{cases}\phi_{1}(r), & \text { for } r_{1}<r<\sqrt{2} r_{c}, r \neq r_{c} \\ \phi_{2}(r), & \text { for } r>r_{c}\end{cases}
$$


where $\phi_{1}(r)$ is

$$
\phi_{1}(r)=\left(\frac{r}{r_{c}}\right)^{-k}\left[a_{1(1)} f_{1(1)}\left(\frac{r^{2}}{r_{c}^{2}}\right)+a_{2(1)} f_{2(1)}\left(\frac{r^{2}}{r_{c}^{2}}\right)\right]
$$

where $f_{1(1)}$ and $f_{2(1)}$ are given by (4.42) and (4.43) with the negative sign of $k$. Function $\phi_{2}(r)$ is given by

$$
\phi_{2}(r)=a_{1(\infty)}\left(\frac{r}{r_{c}}\right)^{-\sqrt{k^{2}+8}} \sum_{n=0}^{\infty} \frac{\left(\frac{-k+\sqrt{k^{2}+8}}{2}\right)_{n},\left(\frac{k+\sqrt{k^{2}+8}}{2}\right)_{n}}{n !\left(\sqrt{k^{2}+8}+1\right)_{n}}\left(\frac{r^{2}}{r_{c}^{2}}\right)^{-n}, r>r_{c} .
$$

The solution for the steady-state case is plotted in figures 4.4 and 4.5 for a normal mode with wavenumber $k=2$. From the graph it is evident that the amplitude of the wave decreases across the critical layer from $r<r_{c}$ to $r>r_{c}$ where the waves are partially absorbed. 


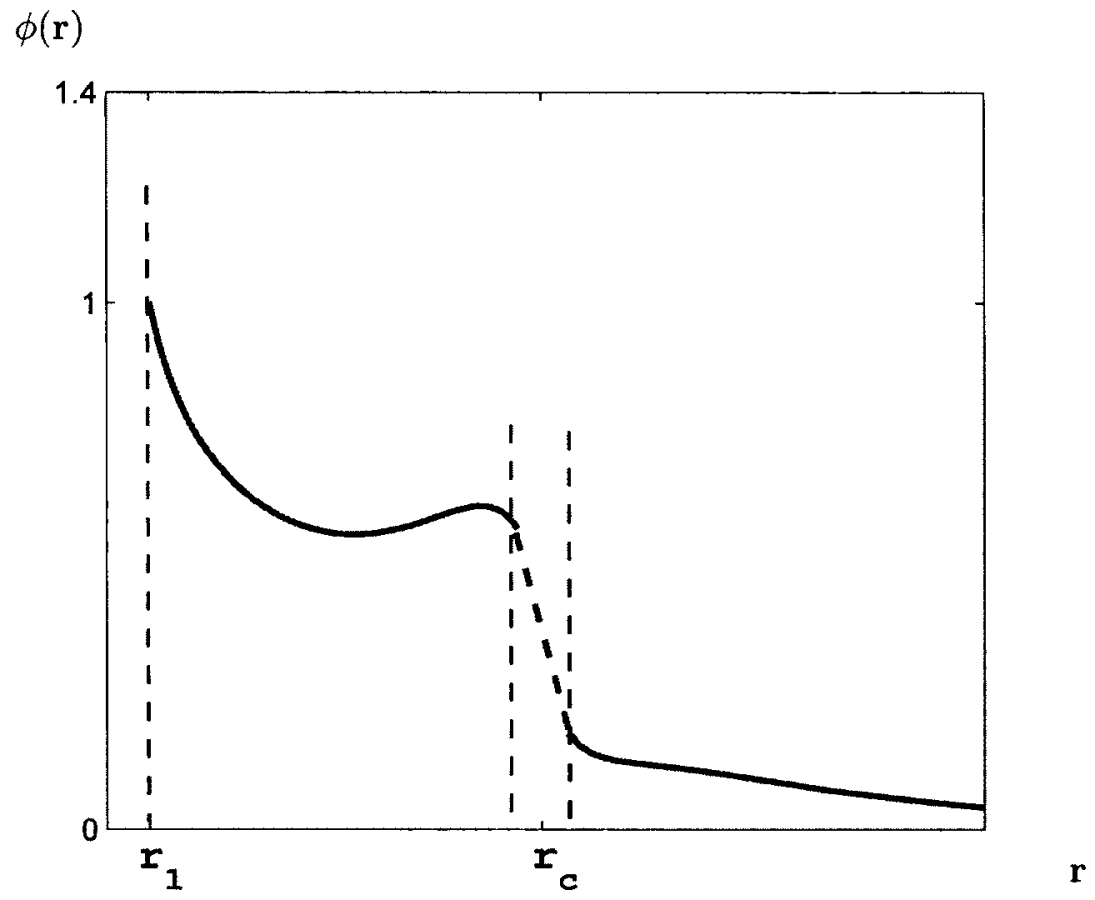

Figure 4.4: The amplitude of the steady solution. Profile of the wave amplitude $\phi(r)$ (4.45) of vortex waves which is the solution of (4.20) with $k=2, a_{1}=1$. The eyewall location $r_{1}$ is taken $r_{1}=0.1 r_{c}$. The dashed line shows that the solution is singular in the inner region close to the critical radius $r_{c}$. 

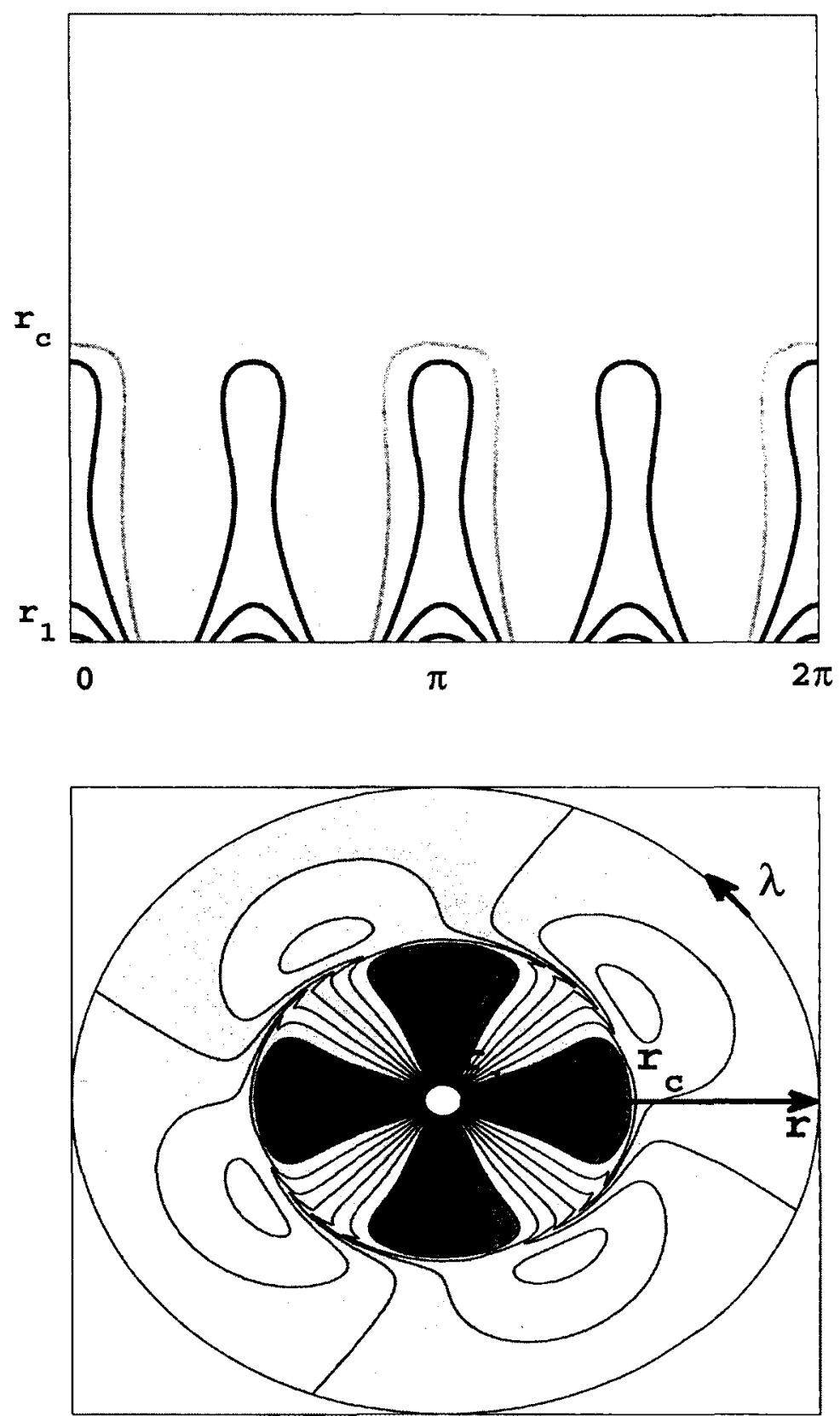

Figure 4.5: Contour plots for the steady solution. Contour plots for the steady solution $\psi(r, \lambda)=\phi(r) e^{i k \lambda}+$ c.c., where the amplitude of vortex waves $\phi(r)(4.45)$ is the solution of (4.20) with $k=2, a_{1}=1$. The eyewall location $r_{1}$ is taken $r_{1}=0.1 r_{c}$. The contour plots are presented in a rectangular domain (the upper panel) and a circular domain (the lower panel) for $r_{1} \leq r<\sqrt{2} r_{c}, 0 \leq \lambda<2 \pi$. The plots demonstrate the phase shift in the solution across the critical level at $r=r_{c}$ and resulting attenuation of the wave amplitude for $r>r_{c}$. 


\section{Chapter 5}

\section{The linear time-dependent problem}

In this chapter and the rest of the thesis we consider the time-dependent problem in which the wave amplitude is allowed to vary with time. In this chapter we solve the linear time-dependent problem on an $f$-plane, i.e. we neglect nonlinearity and the $\beta$-effect. The problem is given by equation (3.54)

$$
\left(\frac{\partial}{\partial t}+\frac{\bar{v}}{r} \frac{\partial}{\partial \lambda}\right) \nabla^{2} \psi-\frac{1}{r} \psi_{\lambda} \frac{\partial}{\partial r}\left(\bar{v}_{r}+\frac{1}{r} \bar{v}\right)=0
$$

in the domain $r_{1} \leq r<\infty, 0 \leq \lambda<2 \pi, t>0$. As in chapter 4 the boundary condition is

$$
\psi\left(r_{1}, \lambda, t\right)=A e^{i(k \lambda-\omega t)}+\text { c.c. },
$$

where $A$ is a constant that we set to 1 . We also require that $\psi(r, \lambda, t)$ and its derivatives be bounded as $r \rightarrow \infty$. The initial condition is that the wave vorticity $\nabla^{2} \psi=0$ at $t=$ 0 . As before, the mean flow is given by the angular velocity profile $\bar{\Omega}(r)=\Omega_{0}\left(1-\alpha r^{2}\right)$ and the azimuthal velocity $\bar{v}(r)=\bar{\Omega}(r) r$. Since the governing equation (5.1) is linear, 
the boundary condition (5.2) means that the solution of (5.1) must take the form

$$
\psi(r, \lambda, t)=\phi(r, t) e^{i k \lambda}+\text { c.c. }
$$

Substituting this into the governing equation (5.1) gives an equation for the wave amplitude $\phi(r, t)$,

$$
\left(\frac{\partial}{\partial t}+i k \bar{\Omega}\right)\left(\phi_{r r}+\frac{1}{r} \phi_{r}-\frac{k^{2}}{r^{2}} \phi\right)+8 i k \Omega_{0} \alpha \phi=0
$$

where the subscripts of $r$ denote partial differentiation with respect to $r$. The boundary conditions are $\phi\left(r_{1}, t\right)=e^{-i \omega t}, \phi(r, t) \rightarrow 0$ as $r \rightarrow \infty$ and the initial condition is $r \phi_{r}+r^{2} \phi_{r r}-k^{2} \phi=0$ at $t=0$. We solve this problem by making use of a Laplace transform.

\subsection{Laplace transform of the linear time-dependent equation}

Let $\tilde{\phi}(r, s)$ be the Laplace transform of the function $\phi(r, t)$, defined as

$$
\tilde{\phi}(r, s)=\int_{0}^{\infty} \phi(r, t) e^{-s t} d t
$$

where $s$ is a complex variable. Taking the Laplace transform of (5.4) and applying the zero initial condition on the vorticity we obtain

$$
(s+i k \bar{\Omega})\left(\tilde{\phi}_{r r}+\frac{1}{r} \tilde{\phi}_{r}-\frac{k^{2}}{r^{2}} \tilde{\phi}\right)+8 i k \Omega_{0} \alpha \tilde{\phi}=0
$$


The boundary condition for $\tilde{\phi}(r, s)$ is

$$
\tilde{\phi}\left(r_{1}, s\right)=\int_{0}^{\infty} e^{-i \omega t} e^{-s t} d t=\frac{1}{s+i \omega}
$$

We can rewrite equation (5.6) in the form

$$
\left(s+i k \Omega_{0}-i k \Omega_{0} \alpha r^{2}\right)\left(\tilde{\phi}_{r r}+\frac{1}{r} \tilde{\phi}_{r}-\frac{k^{2}}{r^{2}} \tilde{\phi}\right)+8 i k \Omega_{0} \alpha \tilde{\phi}=0
$$

Using the fact that $\omega=k \bar{\Omega}\left(r_{c}\right)=k \Omega_{0}\left(1-\alpha r_{c}^{2}\right)$, we can also write (5.8) in terms of $\omega$

$$
\left(s+i \omega+i k \Omega_{0} \alpha\left(r_{c}^{2}-r^{2}\right)\right)\left(\tilde{\phi}_{r r}+\frac{1}{r} \tilde{\phi}_{r}-\frac{k^{2}}{r^{2}} \tilde{\phi}\right)+8 i k \Omega_{0} \alpha \tilde{\phi}=0
$$

Following the same procedure we used for the steady-state case in chapter 4 we introduce a new variable $\hat{r}$, which now depends on $s$,

$$
\hat{r}(s)=\sqrt{\frac{i k \Omega_{0} \alpha}{s+i k \Omega_{0}}} r=\sqrt{\gamma(s)} r
$$

where

$$
\gamma(s)=\frac{i \Omega_{0} \alpha k}{s+i \Omega_{0} k}
$$

We also define $\hat{\phi}(\hat{r}, s)=\tilde{\phi}(r, s)$. In terms of the wave angular frequency $\omega$, we can write

$$
\gamma(s)=\frac{i \Omega_{0} \alpha k}{s+i \omega+i \Omega_{0} k \alpha r_{c}^{2}}
$$

Note that for $s=-i \omega$, we obtain

$$
\gamma(-i \omega)=\frac{1}{r_{c}^{2}}
$$


In terms of the new variables $\hat{r}$ and $\hat{\phi}$, we can rewrite equation (5.8) as

$$
\hat{\phi}_{\hat{r} \hat{r}}+\frac{1}{\hat{r}} \hat{\phi}_{\hat{r}}-\frac{k^{2}}{\hat{r}^{2}} \hat{\phi}-\frac{8}{\hat{r}^{2}-1} \hat{\phi}=0
$$

where the subscripts denote partial derivatives of $\hat{\phi}$ with respect to $\hat{r}$.

Equation (5.14) has the same form as the steady-state equation (4.25) and the solution is of the same form, $\hat{\phi}(\hat{r})=\hat{r}^{p} f\left(\hat{r}^{2}\right)$ with $p=-k$, as for the steady-state case. We have to consider different regions in the complex $s$-plane to find the solution of this equation and evaluate the inverse Laplace transform. Equation (5.14) has three singularities.

- $\hat{r}=0$, or $\frac{i \Omega_{0} \alpha k}{s+i \Omega_{0} k} r^{2}=0$. This case corresponds to $r \rightarrow 0$ and we do not consider it because it is not in the domain of our problem.

- $\hat{r}=1$. Near this singular point, the solution is proportional to a linear combination of $f_{1(1)}\left(\hat{r}^{2}\right)$ and $f_{2(1)}\left(\hat{r}^{2}\right)$, where $f_{1(1)}$ and $f_{2(1)}$ are given by (A.15) and (A.16) in Appendix A. So the solution is

$$
\tilde{\phi}(r, s)=(\sqrt{\gamma(s)} r)^{-k}\left[a_{1(1)}(s) f_{1(1)}\left(\gamma(s) r^{2}\right)+a_{2(1)}(s) f_{2(1)}\left(\gamma(s) r^{2}\right)\right]
$$

where $a_{1(1)}$ and $a_{2(1)}$ are functions of $s$ that can be determined from the boundary conditions. This solution is valid for $0<\left|1-\hat{r}^{2}\right|<1$ (see Abramowitz and Stegun, 1964) which corresponds to the region

$$
0<\left|\frac{i \Omega_{0} \alpha k}{s+i \Omega_{0} k} r^{2}\right|<2,\left|\frac{i \Omega_{0} \alpha k}{s+i \Omega_{0} k} r^{2}\right| \neq 1
$$

or

$$
\left|s+i \Omega_{0} k\right|>\frac{1}{2} \Omega_{0} \alpha k r^{2}, r \neq r_{c}
$$


In the complex $s$-plane this is the region in the complex $s$-plane outside the circle of radius $\frac{1}{2} \Omega_{0} \alpha k r^{2}$ centred at $s=-i \Omega_{0} k$, as shown in Figures 5.1 and 5.2. We shall refer to this as region I.

- $\hat{r} \rightarrow \infty$. For $\hat{r} \gg 1$ the solution of equation (5.14) is proportional to a linear combination of the two functions $f_{1(\infty)}\left(\hat{r}^{2}\right)$ and $f_{2(\infty)}\left(\hat{r}^{2}\right)$, where $f_{1(\infty)}$ and $f_{2(\infty)}$ are given by two functions (A.17) and (A.18) in Appendix A. So the solution is

$$
\tilde{\phi}(r, s)=(\sqrt{\gamma(s)} r)^{-k}\left[a_{1(\infty)}(s) f_{1(\infty)}\left(\gamma(s) r^{2}\right)+a_{2(\infty)}(s) f_{2(\infty)}\left(\gamma(s) r^{2}\right)\right]
$$

where $a_{1(\infty)}$ and $a_{2(\infty)}$ are functions of $s$ that can be determined by matching this solution to $(5.15)$.

This solution is valid for $\left|\hat{r}^{2}\right|=\left|\gamma(s) r^{2}\right|>2$ (see Abramowitz and Stegun, 1964).

$$
\left|s+i \Omega_{0} k\right|<\frac{1}{2} \Omega_{0} \alpha k r^{2} .
$$

This is the region in the complex $s$-plane inside the circle of radius $\frac{1}{2} \Omega_{0} \alpha k r^{2}$ centred at $s=-i \Omega_{0} k$ (the grey shaded region in Figures 5.1 and 5.2). We shall refer to this as region II.

To obtain $\phi(r, t)$ we need to compute the inverse Laplace transform of $\tilde{\phi}(r, s)$. In doing so, we have to take into account the fact that there are two different regions in the complex $s$-plane that need to be considered separately. Region I is outside of the circle $\left|s+i \Omega_{0} k\right|>\frac{1}{2} \Omega_{0} \alpha k r^{2}$ where the solution has the form (5.15), and region II is the region inside the circle $\left|s+i \Omega_{0} k\right|<\frac{1}{2} \Omega_{0} \alpha k r^{2}$ where the solution has the form (5.18) (see Figures 5.1 and 5.2). 

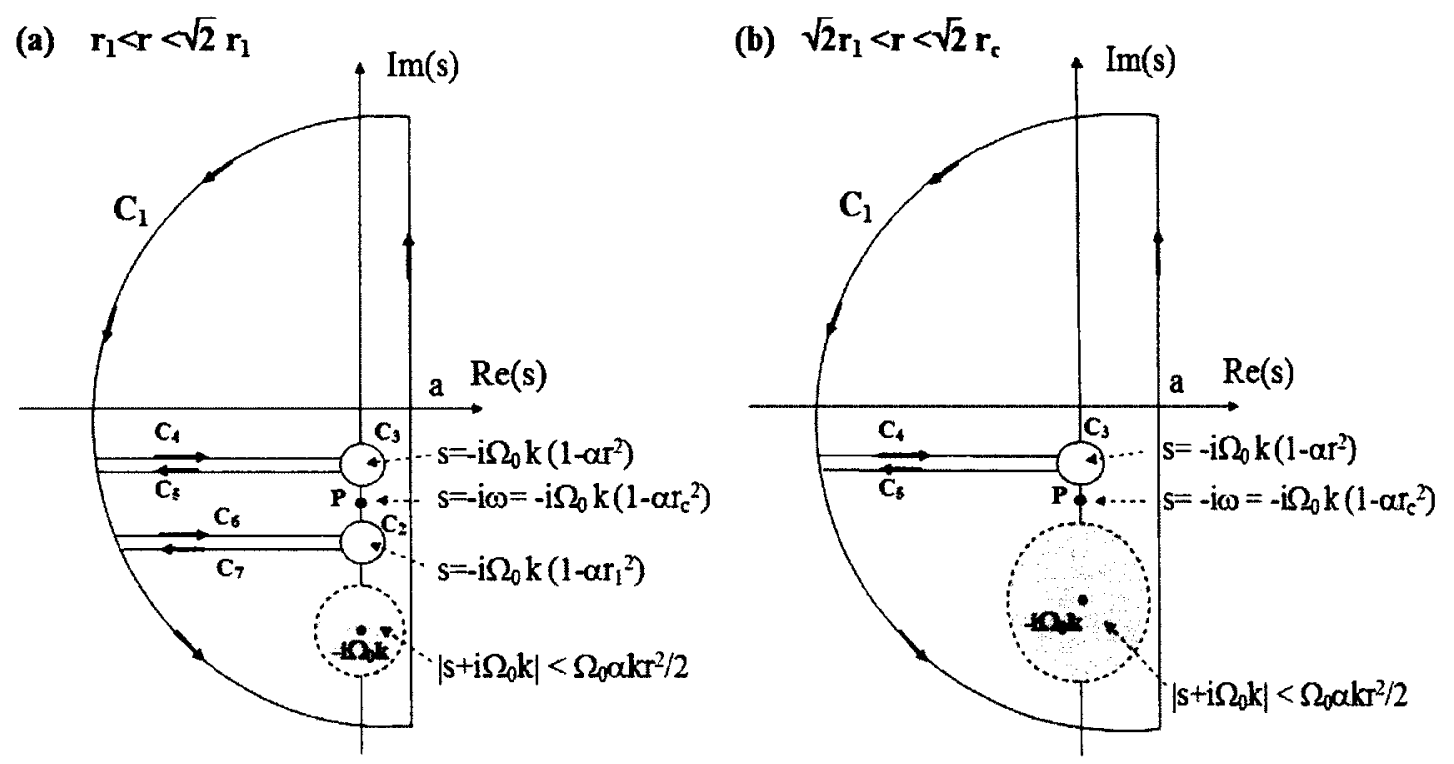

Figure 5.1: The contour of integration in the complex $s$-plane for the inverse Laplace transform for the cases where the pole is in region $I$.

The shaded region is region II where the solution (5.40) is valid. The region outside the circle is region I where the solution (5.39) is valid. The pole $P, s=-i \omega$, is in region I. It gives the steady term of the time-dependent solution in the form (4.59), which is valid for $r_{1}<r<\sqrt{2} r_{c}, r \neq r_{c}$.

(a) If $r>\sqrt{2} r_{1}$, the solution (5.39) has two branch points, $s=-i \Omega_{0} k\left(1-\alpha r^{2}\right)$ and $s=-i \Omega_{0} k\left(1-\alpha r_{1}^{2}\right)$, which are surrounded by the contours $C_{3}$ and $C_{2}$.

(b) If $\sqrt{2} r_{1}<r<\sqrt{2} r_{c}, r \neq r_{c}$, the solution (5.39) has one branch point in region I, $s=-i \Omega_{0} k\left(1-\alpha r^{2}\right)$, surrounded by contour $C_{3}$. 


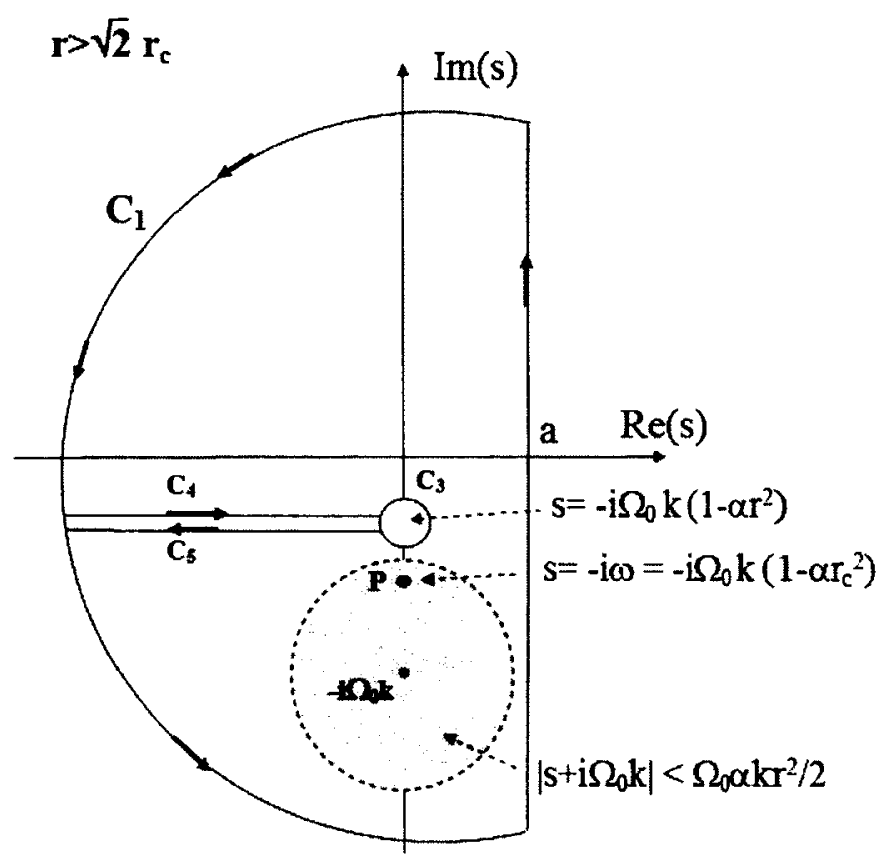

Figure 5.2: The contour of integration in the complex $s$-plane for the inverse Laplace transform for the case where the pole is in region II.

The shaded region is region II where the solution (5.40) is valid. The region outside this disc is region I where the solution (5.39) is valid.

The pole $P, s=-i \omega$, is in region II. It gives the steady term in the time-dependent solution in the form (4.60), which is valid for $r>\sqrt{2} r_{c}$. There is one branch point, in region $\mathrm{I}, s=-i \Omega_{0} k\left(1-\alpha r^{2}\right)$, surrounded by contour $C_{3}$. 


\section{Region I}

In region I (outside the grey disc in Figures 5.1 and 5.2), where $0<\left|1-\gamma(s) r^{2}\right|<1$, the solution is proportional to a linear combination of the two linearly independent solutions (see Appendix A, A.15 and A.16):

$$
f_{1(1)}\left(\gamma(s) r^{2}\right)=\left(1-\gamma(s) r^{2}\right) \sum_{n=0}^{\infty} \frac{\left(\frac{-k+\sqrt{k^{2}+8}}{2}+1\right)_{(n)}\left(\frac{-k-\sqrt{k^{2}+8}}{2}+1\right)_{(n)}}{(2)_{(n)} n !}\left(1-\gamma(s) r^{2}\right)^{n}
$$

and

$$
\begin{aligned}
f_{2(1)}\left(\gamma(s) r^{2}\right)= & -\frac{1}{2} \\
+ & \left(1-\gamma(s) r^{2}\right) \sum_{n=0}^{\infty} \frac{\left(\frac{-k+\sqrt{k^{2}+8}}{2}+1\right)_{(n)}\left(\frac{-k-\sqrt{k^{2}+8}}{2}+1\right)_{(n)}}{(2)_{(n)} n !} \\
+\left(1-\gamma(s) r^{2}\right) & \sum_{n=1}^{\infty} \frac{\left(\frac{-k+\sqrt{k^{2}+8}}{2}+1\right)_{(n)}\left(\frac{-k-\sqrt{k^{2}+8}}{2}+1\right)_{(n)}}{(2)_{(n)} n !}\left(1-\gamma(s) r^{2}\right)^{n} \\
& \left(\chi\left(\frac{-k+\sqrt{k^{2}+8}}{2}+1+n\right)-\chi\left(\frac{-k+\sqrt{k^{2}+8}}{2}+1\right)\right. \\
+\chi\left(\frac{-k-}{2}+1+n\right)-\chi\left(\frac{-k-\sqrt{k^{2}+8}}{2}+1\right)-\chi(2+n) & +\chi(2)-\chi(n+1)+\chi(1)) .
\end{aligned}
$$

Both series (5.20) and (5.21) converge for all $r$ in the interval $0<\left|1-\gamma(s) r^{2}\right|<1$ (see Abramowitz and Stegun, 1964). The solution is

$$
\tilde{\phi}_{I}(r, s)=(\sqrt{\gamma(s)} r)^{-k}\left[a_{1(1)} f_{1(1)}\left(\gamma r^{2}\right)+a_{2(1)} f_{2(1)}\left(\gamma r^{2}\right)\right]
$$

where $a_{1(1)}$ and $a_{2(1)}$ are functions of $s$ which can be determined by boundary conditions. 
The leading-order terms of $f_{1(1)}$ and $f_{2(1)}$ are

$$
\begin{array}{rr}
f_{1(1)}(r, s) \sim\left(1-\gamma(s) r^{2}\right)+O\left(\left(1-\gamma(s) r^{2}\right)^{2}\right), & \left|1-\gamma(s) r^{2}\right|<1, \\
f_{2(1)}(r, s) \sim-\frac{1}{2}+\left(1-\gamma(s) r^{2}\right) \log \left(1-\gamma(s) r^{2}\right)+O\left(1-\gamma(s) r^{2}\right), \\
& \left|1-\gamma(s) r^{2}\right|<1 .
\end{array}
$$

For small values of $\left|1-\gamma(s) r^{2}\right|$ where $\left|1-\gamma(s) r^{2}\right|<1$, the solution $f_{1(1)} \ll f_{2(1)}$. The leading-order terms for the solution are

$$
\begin{array}{r}
\tilde{\phi}_{I}(r, s) \sim(\sqrt{\gamma(s)} r)^{-k}\left(-\frac{1}{2}+\left(1-\gamma(s) r^{2}\right) \log \left(1-\gamma(s) r^{2}\right)+O\left(1-\gamma(s) r^{2}\right)\right) \\
\left|1-\gamma(s) r^{2}\right|<1 .
\end{array}
$$

After applying the boundary condition $\tilde{\phi}\left(r_{1}, s\right)=\frac{1}{s+i \omega}$, we obtain

$$
\begin{array}{r}
\tilde{\phi}_{I}(r, s) \sim \frac{1}{s+i \omega}\left(\frac{r}{r_{1}}\right)^{-k} \frac{-\frac{1}{2}+\left(1-\gamma(s) r^{2}\right) \log \left(1-\gamma(s) r^{2}\right)+O\left(1-\gamma(s) r^{2}\right)}{-\frac{1}{2}+\left(1-\gamma(s) r_{1}^{2}\right) \log \left(1-\gamma(s) r_{1}^{2}\right)+O\left(1-\gamma(s) r_{1}^{2}\right)} \\
\left|1-\gamma(s) r^{2}\right|<1
\end{array}
$$

This solution has two singular points, at $s=-i \Omega_{0} k\left(1-\alpha r^{2}\right)$ where $1-\gamma(s) r^{2}=0$ and $s=-i \Omega_{0} k\left(1-\alpha r_{1}^{2}\right)$ where $1-\gamma(s) r_{1}^{2}=0$.

\section{Region II}

Region II is the grey shaded disc shown in Figure 5.1, where $\left|\gamma(s) r^{2}\right|>2$, or $\mid s+$ $i \Omega_{0} k \mid<\frac{1}{2} \Omega_{0} \alpha k r^{2}$. The point $s=-i \Omega_{0} k$ is the centre of the disc. At the point $s=-i \Omega_{0} k, \gamma(s) \rightarrow \infty$ and for $s \neq-i \Omega_{0} k, \gamma(s)=\frac{i \Omega_{0} \alpha k}{s+i \Omega_{0} k}$ is finite. 
For $s=-i \Omega_{0} k$ equation (5.6) becomes

$$
\tilde{\phi}_{r r}+\frac{1}{r} \tilde{\phi}_{r}-\frac{k^{2}+8}{r^{2}} \tilde{\phi}=0
$$

and the solution is

$$
\tilde{\phi}\left(r,-i \Omega_{0} k\right)=a_{1} r^{\sqrt{k^{2}+8}}+a_{2} r^{-\sqrt{k^{2}+8}}
$$

where $a_{1}$ and $a_{2}$ are constants. The solution should be finite as $r \rightarrow \infty$, so we must set $a_{1}=0$. To find $a_{2}$ we apply the boundary condition (5.7) at $r=r_{1}$ :

$$
\tilde{\phi}\left(r_{1},-i \Omega_{0} k\right)=\frac{1}{-i \Omega_{0} k+i \omega}=-\frac{1}{i \Omega_{0} \alpha k r_{c}^{2}}
$$

and hence obtain

$$
\tilde{\phi}\left(r,-i \Omega_{0} k\right)=-\frac{1}{i \Omega_{0} \alpha k r_{c}^{2}}\left(\frac{r}{r_{1}}\right)^{-\sqrt{k^{2}+8}} .
$$

Equation (5.27) has no singularities in the domain of our problem, so $s=-i \Omega_{0} k$ is not a singular point for the inverse Laplace transform.

For $s \neq-i k \Omega_{0}$, when $\sqrt{\gamma(s)} r$ is large but finite, the solution of (5.6) has a form similar to the steady-state solution for large $r(4.53)$ :

$$
\begin{array}{r}
\tilde{\phi}_{I I}(r, s)=\frac{1}{s+i \omega}(\sqrt{\gamma(s)} r)^{-k}\left[a_{1(\infty)}(s) f_{1(\infty)}\left(\gamma(s) r^{2}\right)+a_{2(\infty)}(s) f_{2(\infty)}\left(\gamma(s) r^{2}\right)\right] \\
\left|\gamma(s) r^{2}\right|>2,
\end{array}
$$

where $a_{1(\infty)}$ and $a_{2(\infty)}$ are functions of $s$, and $f_{1(\infty)}$ and $f_{2(\infty)}$ are the functions given by (A.17) and (A.18) in Appendix A:

$$
\begin{array}{r}
f_{1(\infty)}\left(\gamma(s) r^{2}\right)=\left(\gamma(s) r^{2}\right)^{\frac{k-\sqrt{k^{2}+8}}{2}} \sum_{n=0}^{\infty} \frac{\left(\frac{-k+\sqrt{k^{2}+8}}{2}\right)_{n},\left(\frac{k+\sqrt{k^{2}+8}}{2}\right)_{n}}{n !\left(\sqrt{k^{2}+8}+1\right)_{n}}(\sqrt{\gamma(s)} r)^{-n} \\
\left|\gamma(s) r^{2}\right|>2
\end{array}
$$




$$
\begin{array}{r}
f_{2(\infty)}\left(\gamma(s) r^{2}\right)=\left(\gamma(s) r^{2}\right)^{\frac{k+\sqrt{k^{2}+8}}{2}} \sum_{n=0}^{\infty} \frac{\left(\frac{-k-\sqrt{k^{2}+8}}{2}\right)_{n},\left(\frac{k-\sqrt{k^{2}+8}}{2}\right)_{n}}{n !\left(-\sqrt{k^{2}+8}+1\right)_{n}}(\sqrt{\gamma(s)} r)^{-n} \\
\left|\gamma(s) r^{2}\right|>2 .
\end{array}
$$

So in region II the solution $\tilde{\phi}_{I I}(r, s)$ is a linear combination of the functions

$$
\begin{array}{r}
(\sqrt{\gamma(s)} r)^{-k} f_{1(\infty)}\left(\gamma(s) r^{2}\right) \sim(\sqrt{\gamma(s)} r)^{-k}\left(\gamma(s) r^{2}\right)^{\frac{k-\sqrt{k^{2}+8}}{2}} \sim(\sqrt{\gamma(s)} r)^{-\sqrt{k^{2}+8}} \\
\left|\gamma(s) r^{2}\right|>2
\end{array}
$$

and

$$
\begin{array}{r}
(\sqrt{\gamma(s)} r)^{-k} f_{2(\infty)}\left(\gamma(s) r^{2}\right) \sim(\sqrt{\gamma(s)} r)^{-k}\left(\gamma(s) r^{2}\right)^{\frac{k+\sqrt{k^{2}+8}}{2}} \sim(\sqrt{\gamma(s)} r)^{+\sqrt{k^{2}+8}} \\
\left|\gamma(s) r^{2}\right|>2
\end{array}
$$

The function (5.34) is divergent for $r \rightarrow \infty$. So the coefficient $a_{2(\infty)}(s)=0$ and the solution $(5.30)$ is

$$
\begin{aligned}
& \tilde{\phi}_{I I}(r, s) \sim(\sqrt{\gamma(s)} r)^{-k} a_{1(\infty)} f_{1(\infty)}\left(\gamma(s) r^{2}\right) \\
& \sim a_{1(\infty)}(\sqrt{\gamma(s)} r)^{+\sqrt{k^{2}+8}} \sum_{n=0}^{\infty} \frac{\left(\frac{-k-\sqrt{k^{2}+8}}{2}\right)_{n},\left(\frac{k-\sqrt{k^{2}+8}}{2}\right)_{n}}{n !\left(-\sqrt{k^{2}+8}+1\right)_{n}}\left(\gamma(s) r^{2}\right)^{-n} \\
& \sim a_{1(\infty)}\left(\sqrt{\frac{s+i k \Omega_{0}}{i k \alpha \Omega_{0}} \frac{1}{r}}\right)^{\sqrt{k^{2}+8}} \sum_{n=0}^{\infty} \frac{\left(\frac{-k-\sqrt{k^{2}+8}}{2}\right)_{n},\left(\frac{k-\sqrt{k^{2}+8}}{2}\right)_{n}}{n !\left(-\sqrt{k^{2}+8}+1\right)_{n}}\left(\frac{s+i k \Omega_{0}}{i k \alpha \Omega_{0}} \frac{1}{r^{2}}\right)^{n} \\
& \left|s+i k \Omega_{0}\right|<\frac{1}{2} k \Omega_{0} \alpha r^{2}
\end{aligned}
$$


After applying the boundary condition (5.7) we obtain

$$
\begin{aligned}
& \tilde{\phi}_{I I}(r, s)=\frac{1}{s+i \omega}\left(\frac{r}{r_{1}}\right)^{-\sqrt{k^{2}+8}} \frac{\sum_{n=0}^{\infty} \frac{\left.\frac{\left(-k-\sqrt{k^{2}+8}\right.}{2}\right)_{n},\left(\frac{k-\sqrt{k^{2}+8}}{2}\right)_{n}}{n !\left(-\sqrt{k^{2}+8}+1\right)_{n}}\left(\frac{s+i k \Omega_{0}}{i k \alpha \Omega_{0}} \frac{1}{r^{2}}\right)^{n}}{\sum_{n=0}^{\infty} \frac{\left(\frac{-k-\sqrt{k^{2}+8}}{2}\right)_{n},\left(\frac{k-\sqrt{k^{2}+8}}{2}\right)_{n}}{n !\left(-\sqrt{k^{2}+8}+1\right)_{n}}\left(\frac{s+i k \Omega_{0}}{i k \alpha \Omega_{0}} \frac{1}{r_{1}^{2}}\right)^{n}}, \\
& \left|s+i k \Omega_{0}\right|<\frac{1}{2} k \Omega_{0} \alpha r^{2} .
\end{aligned}
$$

The leading-order terms of the solution are

$$
\begin{array}{r}
\tilde{\phi}_{I I}(r, s) \sim \frac{1}{s+i \omega}\left(\frac{r}{r_{1}}\right)^{-\sqrt{k^{2}+8}}\left(1+O\left(\frac{s+i k \Omega_{0}}{i k \alpha \Omega_{0}} \frac{1}{r^{2}}\right)+O\left(\frac{s+i k \Omega_{0}}{i k \alpha \Omega_{0}} \frac{1}{r_{1}^{2}}\right)\right) \\
\text { for }\left|s+i k \Omega_{0}\right|<\frac{1}{2} k \Omega_{0} \alpha r^{2}
\end{array}
$$

We observe that this solution gives us (5.29) in the limit $s \rightarrow-i \Omega_{0} k$. So the solution $\tilde{\phi}_{I I}(r, s)$ is continuous in region II where it is defined. We have now obtained the solution $\tilde{\phi}(r, s)$ everywhere in the complex $s$-space and can find $\phi(r, t)$ by making use of an inverse Laplace transform.

\subsection{Evaluation of the inverse Laplace transform}

In section 5.1 we derived the solution of equation (5.6) in different regions in complex space,

$$
\tilde{\phi}(r, s)= \begin{cases}\tilde{\phi}_{I}(r, s), & \text { for }\left|s+i k \Omega_{0}\right|>\frac{1}{2} k \Omega_{0} \alpha r^{2} \text { (Region I), } \\ \tilde{\phi}_{I I}(r, s), & \text { for }\left|s+i k \Omega_{0}\right|<\frac{1}{2} k \Omega_{0} \alpha r^{2} \text { (Region II), }\end{cases}
$$


where

$$
\begin{gathered}
\tilde{\phi}_{I}(r, s) \sim \frac{1}{s+i \omega}\left(\frac{r}{r_{1}}\right)^{-k} \frac{-\frac{1}{2}+\left(1-\gamma(s) r^{2}\right) \log \left(1-\gamma(s) r^{2}\right)+O\left(\left(1-\gamma(s) r^{2}\right)\right)}{-\frac{1}{2}+\left(1-\gamma(s) r_{1}^{2}\right) \log \left(1-\gamma(s) r_{1}^{2}\right)+O\left(\left(1-\gamma(s) r_{1}^{2}\right)\right)} \\
\sim \frac{1}{s+i \omega}\left(\frac{r}{r_{1}}\right)^{-k} \frac{1-2\left(1-\frac{i k \Omega_{0} \alpha}{s+i k \Omega_{0}} r^{2}\right) \log \left(1-\frac{i k \Omega_{0} \alpha}{s+i k \Omega_{0}} r^{2}\right)+O\left(\left(1-\frac{i k \Omega_{0} \alpha}{s+i k \Omega_{0}} r^{2}\right)\right)}{1-2\left(1-\frac{i k \Omega_{0} \alpha}{s+i k \Omega_{0}} r_{1}^{2}\right) \log \left(1-\frac{i k \Omega_{0} \alpha}{s+i k \Omega_{0}} r_{1}^{2}\right)+O\left(\left(1-\frac{i k \Omega_{0} \alpha}{s+i k \Omega_{0}} r_{1}^{2}\right)\right)},
\end{gathered}
$$

and

$$
\tilde{\phi}_{I I}(r, s) \sim \frac{1}{s+i \omega}\left(\frac{r}{r_{1}}\right)^{-\sqrt{k^{2}+8}}\left(1+O\left(\frac{s+i k \Omega_{0}}{i k \alpha \Omega_{0}} \frac{1}{r^{2}}\right)+O\left(\frac{s+i k \Omega_{0}}{i k \alpha \Omega_{0}} \frac{1}{r_{1}^{2}}\right)\right)
$$

To find $\phi(r, t)$, the solution of equation (5.1), we evaluate the inverse Laplace transform of $\tilde{\phi}(r, s)$ using the Laplace inversion integral

$$
\phi(r, t)=\frac{1}{2 \pi i} \int_{a-i \infty}^{a+i \infty} \frac{1}{s+i \omega} \tilde{\phi}(r, s) e^{s t} d s
$$

where $\tilde{\phi}(r, s)$ is given by (5.38). Here $a$ is a real number chosen so that the contour of integration will lie to the right of all the singularities of the integrand. The integral is evaluated along the closed Bromwich contour in the complex $s$-plane shown in Figures 5.1 and 5.2. The contour of integration is closed around the pole of the integrand and deformed to go around any branch points of the integrand. Following the standard procedure, the radius of the large semicircle is allowed to become infinite while the radii of the circles around the branch points go to zero. Making use of the residue theorem the integral is found to be equal to the sum of the contributions from the singularities.

We evaluate the inverse Laplace transform in the complex $s$-plane for each value of $r, r_{1}<r<\infty$. Depending on the value of $r$, we have three different configurations in the complex $s$-plane. Case 1 for $r_{1}<r<\sqrt{2} r_{1}$, Case 2 for $\sqrt{r_{1}}<r<\sqrt{2} r_{c}, r \neq r_{c}$, 
and Case 3 for $r>r_{c}$. The union of the intervals in Case 1 and Case 2 is the interval $r_{1}<r<\sqrt{2} r_{c}, r \neq r_{c}$, which is where the steady solution (4.59) is valid. And we shall see that in the limit as $t \rightarrow \infty$ the time-dependent solution that is valid in this interval converges to the steady solution (4.59). The interval in Case 3 is $r>\sqrt{2} r_{c}$ which is where the steady solution (4.60) is valid. And we shall see that in the limit as $t \rightarrow \infty$, the time-dependent solution that is valid in this interval converges to the steady solution (4.60). The three cases are summarized below and are represented in Figures 5.1 and 5.2.

Case 1. $r_{1}<r<\sqrt{2} r_{1}$

In this case the integrand in (5.41) has

- a pole $P$ at $s=-i \omega$ in region $\mathrm{I}$, outside the grey disc,

- a branch point at $s=-i \Omega_{0} k\left(1-\alpha r^{2}\right)$, or $s=-i \omega+i k \Omega_{0} \alpha\left(r^{2}-r_{c}^{2}\right)$,

- a branch point at $s=-i \Omega_{0} k\left(1-\alpha r_{1}^{2}\right)$, or $s=-i \omega+i k \Omega_{0} \alpha\left(r_{1}^{2}-r_{c}^{2}\right)$.

All the points lie outside the grey disc, so they are in region I. This is shown in Figure 5.1a.

Case 2. $\sqrt{2} r_{1}<r<\sqrt{2} r_{c}, r \neq r_{c}$

In this case the integrand in (5.41) has

- a pole $P$ at $s=-i \omega$ in region $\mathrm{I}$, outside the grey disc,

- a branch point at $s=-i \Omega_{0} k\left(1-\alpha r^{2}\right)$.

The point $\left(s=-i \Omega_{0} k\left(1-\alpha r_{1}^{2}\right)\right)$, in this case, lies inside the grey disc, i.e. in region II. Since it is not in region 1 , it does not contribute to the solution. This is shown in Figure 5.1b. 
Case 3. $r>\sqrt{2} r_{c}$

In this case the integrand in (5.41) has

- a pole $P$ at $s=-i \omega$ which is now in the region II,

- a branch point of the solution (5.39) at $s=-i \Omega_{0} k\left(1-\alpha r^{2}\right)$.

As in case 2 , the point $\left(s=-i \Omega_{0} k\left(1-\alpha r_{1}^{2}\right)\right)$ lies inside the grey disc, i.e. in region II. Since it is not in region I, it does not contribute to the solution. This is shown in Figure 5.2.

We now describe the evaluation of the contributions from the pole and branch points.

\section{The residue at the pole $P, s=-i \omega$}

In all 3 cases the integrand in (5.41) has a pole at $s=-i \omega=-i \Omega_{0} k\left(1-\alpha r_{c}^{2}\right)$. The residue at the pole is

$$
\operatorname{Res}_{(s=-i \omega)}=\lim _{s \rightarrow-i \omega}\left(\tilde{\phi}(r, s) e^{s t}\right)=\tilde{\phi}(r,-i \omega) e^{-i \omega t} .
$$

For cases 1 and 2, where $r_{1}<r<\sqrt{2} r_{c}$, the pole $P$ is in region I so we use $\tilde{\phi}(r, s)=\tilde{\phi}_{I}(r, s)$ in $(5.42)$. Recalling that $\gamma(-i \omega)=\frac{1}{r_{c}^{2}}(5.13)$, we obtain

$$
\begin{aligned}
& \operatorname{Res}_{(s=-i \omega)}\left(\tilde{\phi}_{I}(r, s)\right)=\operatorname{Res}_{s=-i \omega}\left(\tilde{\phi}_{I}(r, s)\right) \\
& \sim e^{-i \omega t}\left(\frac{r}{r_{1}}\right)^{-k} \frac{-\frac{1}{2}+\left(1-\frac{r^{2}}{r_{c}^{2}}\right) \log \left(1-\frac{r^{2}}{r_{c}^{2}}\right)+O\left(1-\frac{r^{2}}{r_{c}^{2}}\right)}{-\frac{1}{2}+\left(1-\frac{r_{1}^{2}}{r_{c}^{2}}\right) \log \left(1-\frac{r_{1}^{2}}{r_{c}^{2}}\right)+O\left(1-\frac{r_{1}^{2}}{r_{c}^{2}}\right)}
\end{aligned}
$$

For cases 1 and 2 the residue corresponds to the steady-state solution (4.59) which is valid for $r_{1}<r<\sqrt{2} r_{c}, r \neq r_{c}$. For case 3 , where $r>\sqrt{2} r_{c}$, the pole $-i \omega$ is in region 
II (Figure 5.2) so we use $\tilde{\phi}(r, s)=\tilde{\phi}_{I I}(r, s)$ in $(5.42)$ and therefore

$$
\begin{aligned}
& \operatorname{Res}_{(s=-i \omega)}(\tilde{\phi}(r, s))= \operatorname{Res}_{s=-i \omega}\left(\tilde{\phi}_{I I}(r, s)\right) \\
&=\left(\frac{r}{r_{1}}\right)^{-\sqrt{k^{2}+8}} \frac{\sum_{n=0}^{\infty} \frac{\left(\frac{-k-\sqrt{k^{2}+8}}{2}\right)_{n},\left(\frac{k-\sqrt{k^{2}+8}}{2}\right)_{n}}{n !\left(-\sqrt{k^{2}+8}+1\right)_{n}}\left(\frac{r_{c}^{2}}{r^{2}}\right)^{n}}{\sum_{n=0}^{\infty} \frac{\left(\frac{-k-\sqrt{k^{2}+8}}{2}\right)_{n},\left(\frac{k-\sqrt{k^{2}+8}}{2}\right)_{n}}{n !\left(-\sqrt{k^{2}+8}+1\right)_{n}}\left(\frac{r_{c}^{2}}{r_{1}^{2}}\right)^{n}} .
\end{aligned}
$$

This residue corresponds to the steady-state solution which is valid for $r>\sqrt{2} r_{c}$.

\section{The contributions from the branch points}

To evaluate the inverse Laplace transform we integrate (5.39) along the contours shown in Figures 5.1 and 5.2. Let us consider the integral for the case 1 where the integrand has a pole and two branch points. For case 1 the contour consists of the 7 contours $C_{1}-C_{7}$ which are shown in Figure 5.1a.

So the solution is

$$
\phi(r, t)=\operatorname{Res}_{(s=-i \omega)}\left(\frac{1}{s+i \omega} \tilde{\phi}(r, s)\right)-\frac{1}{2 \pi i} \sum_{i=1}^{7} \int_{C_{i}} \frac{1}{s+i \omega} \tilde{\phi}(r, s) e^{s t} d s
$$

\section{The contours $C_{1}, C_{2}$, and $C_{3}$}

The contour $C_{1}$ can be described in polar coordinates as

$$
s=a+R e^{i \theta}, \quad \frac{\pi}{2}<\theta<\frac{3 \pi}{2} .
$$

So

$$
\int_{C_{1}} \frac{1}{s+i \omega} \tilde{\phi}(r, s) e^{s t} d s=\int_{\pi / 2}^{3 \pi / 2} \frac{1}{a+R e^{i \theta}+i \omega} e^{\left(a+R e^{i \theta}\right) t} \tilde{\phi}\left(r, a+R e^{i \theta}\right) i R e^{i \theta} d \theta
$$


As $R \rightarrow \infty$, the integrand in (5.47) is proportional to $e^{R e^{i \theta}}$ or $e^{R \cos \theta}$, and $\cos \theta$ is negative since $\frac{\pi}{2}<\theta<\frac{3 \pi}{2}$. So the integral along the semicircular contour $C_{1}$ converges to zero as the radius of the semicircle becomes infinite.

The contour $C_{2}$ is the circle surrounding the branch point $s=-i \Omega_{0} k\left(1-\alpha r^{2}\right)$. It can be described as

$$
s=-i \Omega_{0} k\left(1-\alpha r^{2}\right)+\epsilon e^{i \theta}, \quad \pi<\theta<3 \pi
$$

So

$$
\begin{aligned}
& \int_{C_{2}} \frac{1}{s+i \omega} \tilde{\phi}(r, s) e^{s t} d s \\
& =\int_{\pi}^{\pi} \frac{\tilde{\phi}\left(r,-i \Omega_{0} k\left(1-\alpha r^{2}\right)+\epsilon e^{i \theta}\right)}{-i \Omega_{0} k\left(1-\alpha\left(r^{2}-r_{c}^{2}\right)\right)+\epsilon e^{i \theta}} e^{-i \Omega_{0} k\left(1-\alpha\left(r^{2}-r_{c}^{2}\right)\right)+\epsilon e^{i \theta}} i \epsilon e^{i \theta} d \theta
\end{aligned}
$$

and the integral converges to zero when $\epsilon \rightarrow 0$.

The contour $C_{3}$ is the circle surrounding the branch point $s=-i \Omega_{0} k\left(1-\alpha r_{1}^{2}\right)$. The integral along $C_{3}$ takes a similar form to (5.49) and converges to zero as the radius of the circle converges to zero.

So the inverse Laplace transform for case 1 is equal to the contribution from the residue at the pole (5.43) plus the contributions from the contours $C_{4}$ and $C_{5}$ near the branch point $s=-i \Omega_{0} k\left(1-\alpha r^{2}\right)$ and from the contours $C_{6}$ and $C_{7}$ near the branch point $s=-i \Omega_{0} k\left(1-\alpha r_{1}^{2}\right)$.

\section{The contours $C_{4}$ and $C_{5}$}

The contours $C_{4}$ and $C_{5}$ correspond to cuts to the branch point $s=-i \Omega_{0} k\left(1-\alpha r^{2}\right)$, or $s=-i \omega+i k \Omega_{0} \alpha\left(r^{2}-r_{c}^{2}\right)$. Along the contour $C_{4}, s=s_{+}=\rho e^{i \pi}-i \omega+i k \Omega_{0} \alpha\left(r^{2}-r_{c}^{2}\right)$, 
and $\rho$ changes from $\infty$ to 0 . Along the contour $C_{5}, s=s_{-}=\rho e^{-i \pi}-i \omega+i k \Omega_{0} \alpha\left(r^{2}-r_{c}^{2}\right)$, and $\rho$ changes from 0 to $\infty$. Hence

$$
\begin{aligned}
& \left(\int_{C_{4}}+\int_{C_{5}}\right)\left(\tilde{\phi}(r, s) e^{s t}\right) d s \sim e^{-i \omega t} e^{i k \Omega_{0} \alpha\left(r^{2}-r_{c}^{2}\right) t}\left(\frac{r}{r_{1}}\right)^{-k} \\
& \quad \int_{0}^{\infty} \frac{1}{(s+i \omega)} \frac{1-2\left(1-\gamma(s) r^{2}\right) \log \left(1-\gamma(s) r^{2}\right)+O\left(1-\gamma(s) r^{2}\right)}{1-2\left(1-\gamma(s) r_{1}^{2}\right) \log \left(1-\gamma(s) r_{1}^{2}\right)+O\left(1-\gamma(s) r_{1}^{2}\right)} e^{-\rho t} d \rho
\end{aligned}
$$

For large $t$, the dominant contribution to this integral comes from small $\rho$ because of the factor of $e^{-\rho t}$, so we can obtain an approximation valid for large $t$. Let us estimate the integrand for small values of $\rho$. We can write

$$
\begin{array}{r}
\frac{1}{s+i \omega}=\frac{1}{i k \Omega_{0} \alpha\left(r+r_{c}\right)\left(r-r_{c}\right)-\rho}=\frac{1}{i k \Omega_{0} \alpha\left(r+r_{c}\right)\left(r-r_{c}\right)} \frac{1}{1-\frac{\rho}{i k \Omega_{0} \alpha\left(r-r_{c}\right)\left(r+r_{c}\right)}} \\
=\frac{1}{i k \Omega_{0} \alpha\left(r+r_{c}\right)\left(r-r_{c}\right)}\left(1-\frac{1}{i k \Omega_{0} \alpha\left(r+r_{c}\right)} \frac{\rho}{r-r_{c}}\right)^{-1}
\end{array}
$$

and expand it as a geometric series

$$
\frac{1}{s+i \omega}=\frac{1}{i k \Omega_{0} \alpha\left(r+r_{c}\right)\left(r-r_{c}\right)}\left[1+\sum_{n=1}^{\infty}\left(c_{n} \frac{\rho}{r-r_{c}}\right)^{n}\right]
$$

This expansion is valid for $\frac{\rho}{\left|r-r_{c}\right|} \ll 1$. The other terms in the integrand (5.50) can also be approximated for small $\rho$. Along the contours $C_{4}$ and $C_{5}, \gamma(s)$ can be expressed in terms of $\rho$ as

$$
\gamma(s)=\frac{i \Omega_{0} \alpha k}{i k \Omega_{0} \alpha r^{2}-\rho}
$$


In the numerator of the integrand in $(5.50)$ we have

$$
\begin{array}{r}
1-\gamma(s) r^{2}=\frac{-\rho}{i k \Omega_{0} \alpha r^{2}-\rho} \sim \frac{-\rho}{i k \Omega_{0} \alpha r^{2}}\left(1+\frac{\rho}{i k \Omega_{0} \alpha r^{2}}+O\left(\rho^{2}\right)\right), \\
\rho \ll 1 .
\end{array}
$$

In the denominator of the integrand in (5.50) we have

$$
1-\gamma(s) r_{1}^{2} \sim\left(\frac{r^{2}-r_{1}^{2}}{r^{2}}+\frac{\rho e^{i \pi}}{i k \Omega_{0} \alpha r^{2}}\right)\left(1-\frac{\rho e^{i \pi}}{i k \Omega_{0} \alpha r^{2}}+O\left(\rho^{2}\right)\right), \rho \ll 1
$$

Thus, the denominator of the integrand in (5.50) is

$$
\begin{aligned}
& 1-2\left(1-\gamma(s) r_{1}^{2}\right) \log \left(1-\gamma(s) r_{1}^{2}\right) \\
& \quad \sim 1-2 \frac{r^{2}-r_{1}^{2}}{r^{2}} \log \left(\frac{r^{2}-r_{1}^{2}}{r^{2}}\right)-2 \frac{\rho e^{i \pi}}{i k \Omega_{0} \alpha} \frac{r_{1}^{2}}{r^{4}}\left(\log \left(\frac{r^{2}-r_{1}^{2}}{r^{2}}\right)+1\right)+ \\
& \quad \begin{array}{l}
O\left(\rho^{2}\right), \\
\rho \ll 1 .
\end{array}
\end{aligned}
$$

The sum of the integrals along $C_{4}$ and $C_{5}$ is proportional to the phase shift between the two branches of the logarithm,

$$
\log \left(1-\gamma\left(s_{+}\right) r^{2}\right)-\log \left(1-\gamma\left(s_{-}\right) r^{2}\right)=-2 \pi i
$$

and the sum of the integrals is

$$
\begin{array}{r}
\left(\int_{C_{4}}+\int_{C_{5}}\right) \tilde{\phi}(r, s) e^{s t} d s \sim e^{-i \omega t} e^{i k \Omega_{0} \alpha\left(r^{2}-r_{c}^{2}\right) t}\left(\frac{r}{r_{1}}\right)^{-k}(-2 i \pi) \\
\int_{0}^{\infty} \sum_{n=1}^{\infty} a_{n}\left(\frac{\rho}{r-r_{c}}\right)^{n} e^{-\rho t} d \rho, \quad \rho \ll 1,
\end{array}
$$


where

$$
\begin{gathered}
a_{1}=\frac{2 h_{1}(r)}{\left(i k \Omega_{0} \alpha\right)^{2}\left(r+r_{c}\right) r^{2}} \\
a_{2}=\frac{h_{2}(r)}{\left(i k \Omega_{0} \alpha\right)^{3}\left(r+r_{c}\right) r^{4}} \\
h_{1}(r)=\left(1-2 \frac{r^{2}-r_{1}^{2}}{r^{2}} \log \left(\frac{r^{2}-r_{1}^{2}}{r^{2}}\right)\right)^{-1}, \\
h_{2}(r)=2 h_{1}(r)\left(r_{c}^{2}-2 r^{2}+2 h_{1}(r) \frac{r_{1}^{1}}{r^{2}}\left(r^{2}-r_{c}^{2}\right)\left(\log \left(\frac{r^{2}-r_{1}^{2}}{r^{2}}\right)+1\right)\right) .
\end{gathered}
$$

The expansion (5.58) is convergent for $\frac{\rho}{\left|r-r_{c}\right|}<1$ and so we can interchange the order of the integration and summation in (5.58). For each $n$, we can express the integral in terms of the gamma function $\Gamma(n)$ as

$$
\int_{0}^{\infty} \rho^{n-1} e^{-\rho t} d \rho=\frac{\Gamma(n)}{t^{n}}
$$

and we evaluate the integral (5.50) along the contours $C_{4}$ and $C_{5}$ as

$$
\begin{aligned}
& \left(\int_{C_{4}}+\int_{C_{5}}\right) \tilde{\phi}(r, s) e^{s t} d s \\
& \sim 2 i \pi e^{-i \omega t} e^{i k \Omega_{0} \alpha\left(r^{2}-r_{c}^{2}\right) t}\left(\frac{r}{r_{1}}\right)^{-k} \frac{1}{t} \sum_{n=1}^{\infty} a_{n} \Gamma(n+1) \frac{1}{\left(t\left(r-r_{c}\right)\right)^{n}}, \text { for }\left|r-r_{c}\right| t>1 .(5 .
\end{aligned}
$$

\section{The contours $C_{6}$ and $C_{7}$}

The contours $C_{6}$ and $C_{7}$ correspond to cuts to the branch point $s=-i \Omega_{0} k\left(1-\alpha r_{1}^{2}\right)$, or $s=-i \omega-i \Omega_{0} \alpha k\left(r_{c}^{2}-r_{1}^{2}\right)$. Along the contour $C_{6}, s=s_{+}=\rho e^{i \pi}-i \Omega_{0} k\left(1-\alpha r_{1}^{2}\right)$, and $\rho$ changes from $\infty$ to 0 . Along the contour $C_{7}, s=s_{-}=\rho e^{-i \pi}-i \Omega_{0} k\left(1-\alpha r_{1}^{2}\right)$, 
and $\rho$ changes from 0 to $\infty$. Hence

$$
\begin{aligned}
& \left(\int_{C_{6}}+\int_{C_{7}}\right) \tilde{\phi}(r, s) e^{s t} d s \sim e^{-i \omega t} e^{i k \Omega_{0} \alpha\left(r_{1}^{2}-r_{c}^{2}\right) t}\left(\frac{r}{r_{1}}\right)^{-k} \\
& \quad \int_{0}^{\infty} \frac{1}{(s+i \omega)} \frac{1-2\left(1-\gamma(s) r^{2}\right) \log \left(1-\gamma(s) r^{2}\right)+O\left(1-\gamma(s) r^{2}\right)}{1-2\left(1-\gamma(s) r_{1}^{2}\right) \log \left(1-\gamma(s) r_{1}^{2}\right)+O\left(1-\gamma(s) r_{1}^{2}\right)} e^{-\rho t} d \rho
\end{aligned}
$$

The dominant contribution to the integrand comes from small $\rho$ because of the factor of $e^{-\rho t}$. So for $t \gg 1$ we can approximate the integrand by considering small $\rho$. The term $\frac{1}{s+i \omega}$ in the integral is approximated by

$$
\frac{1}{s+i \omega} \sim \frac{1}{i k \Omega_{0} \alpha\left(r_{1}^{2}-r_{c}^{2}\right)}\left(1-\frac{\rho}{i k \Omega_{0} \alpha\left(r_{c}^{2}-r_{1}^{2}\right)}+O\left(\rho^{2}\right)\right)
$$

Along the contours $C_{6}$ and $C_{7}, \gamma(s)$ is written in terms of $\rho$ as

$$
\gamma=\frac{i \Omega_{0} \alpha k}{i k \Omega_{0} \alpha r_{1}^{2}-\rho}
$$

and for small $\rho$ it can be approximated as

$$
\gamma(s)=\frac{1}{r_{1}^{2}\left(1+\rho e^{i \pi} / i k \Omega_{0} \alpha r_{1}^{2}\right)} \sim \frac{1}{r_{1}^{2}}\left(1-\rho / i k \Omega_{0} \alpha r^{2}\right)+O\left(\rho^{2}\right)
$$

We also make the approximation

$$
\left(1-\gamma(s) r^{2}\right) \sim\left(\frac{r_{1}^{2}-r^{2}}{r_{1}^{2}}+\frac{-\rho}{i k \Omega_{0} \alpha r_{1}^{2}}\right)\left(1+\frac{-\rho}{i k \Omega_{0} \alpha r_{1}^{2}}+O\left(\rho^{2}\right)\right)
$$

So the numerator of the integrand (5.64) becomes

$$
\begin{aligned}
& 1-2\left(1-\gamma(s) r^{2}\right) \log \left(1-\gamma(s) r^{2}\right) \sim \\
& 1-2 \frac{r_{1}^{2}-r^{2}}{r_{1}^{2}} \log \left(\frac{r_{1}^{2}-r^{2}}{r_{1}^{2}}\right)+2 \frac{\rho}{i k \Omega_{0} \alpha} \frac{r^{2}}{r_{1}^{4}}\left(\log \left(\frac{r_{1}^{2}-r^{2}}{r_{1}^{2}}\right)+1\right)+O\left(\rho^{2}\right), \rho \ll 1(5.60)
\end{aligned}
$$


The sum of the integrals along the contours $C_{6}$ and $C_{7}$ depends on the phase shift between two branches of the logarithm. So again we have

$$
\log \left(1-\gamma\left(s_{+}\right) r_{1}^{2}\right)-\log \left(1-\gamma\left(s_{-}\right) r_{1}^{2}\right)=-2 \pi i
$$

So for small $\rho$ we can write the leading-order terms of the sum of the integrals along $C_{6}$ and $C_{7}$ in the form

$$
\begin{aligned}
& \left(\int_{C_{6}}+\int_{C_{7}}\right) \tilde{\phi}(r, s) e^{s t} d s \sim \\
& \quad e^{-i \omega t} e^{i k \Omega_{0} \alpha\left(r_{1}^{2}-r_{c}^{2}\right) t}\left(\frac{r}{r_{1}}\right)^{-k}(-2 i \pi) \int_{0}^{\infty}\left(\sum_{n=1}^{\infty} b_{n} \rho^{n}\right) e^{-\rho t} d \rho, \rho \ll 1,
\end{aligned}
$$

where

$$
b_{1}(r)=\frac{h_{3}(r)}{\left(i k \Omega_{0} \alpha\right)^{2}\left(r_{1}^{2}-r_{c}^{2}\right) r_{1}^{2}}
$$

with

$$
h_{3}(r)=2\left(1-2 \frac{r_{1}^{2}-r^{2}}{r_{1}^{2}} \log \left(\frac{r_{1}^{2}-r^{2}}{r_{1}^{2}}\right)\right)^{-1}
$$

So the sum of integrals along $C_{6}$ and $C_{7}$ is

$$
\left(\int_{C_{6}}+\int_{C_{7}}\right) \tilde{\phi}(r, s) e^{s t} d s \sim-2 i \pi e^{-i \omega t} e^{i k \Omega_{0} \alpha\left(r^{2}-r_{c}^{2}\right) t}\left(\frac{r}{r_{1}}\right)^{-k}\left(\sum_{n=2}^{\infty} b_{i} \frac{\Gamma(n)}{t^{n}}\right), t \gg
$$

So we have obtained approximations for the time-dependent solution, valid for $t \gg 1$, by evaluating the inverse Laplace transform in each of the cases shown in Figures 5.1 and 5.2 . 
Case 1, $r_{1}<r<\sqrt{2} r_{1}$

For $r_{1}<r<\sqrt{2} r_{1}$, the configuration for the inversion of the Laplace transform in the complex s-plane is shown in Figure 5.1a. The pole is in region I and there are two branch points. In this case the solution is

$$
\begin{aligned}
& \phi(r, t) \sim \Phi_{\infty}(r) e^{-i \omega t} \\
& +e^{-i \omega t} e^{i k \Omega_{0} \alpha\left(r^{2}-r_{c}^{2}\right) t}\left(\frac{r}{r_{1}}\right)^{-k}\left(\frac{2 h_{1}(r)}{t^{2}\left(i k \Omega_{0} \alpha\right)^{2}\left(r^{2}-r_{c}^{2}\right) r^{2}}+\frac{2 h_{2}(r)}{t^{3}\left(i k \Omega_{0} \alpha\right)^{3}\left(r^{2}-r_{c}^{2}\right)^{2} r^{4}}\right) \\
& +e^{-i \omega t} e^{i k \Omega_{0} \alpha\left(r_{1}^{2}-r_{c}^{2}\right) t}\left(\frac{r}{r_{1}}\right)^{-k}\left(\frac{h_{3}(r)}{t^{2}\left(i k \Omega_{0} \alpha\right)^{2}\left(r_{1}^{2}-r_{c}^{2}\right) r_{1}^{2}}+\frac{h_{2}(r)}{t^{3}\left(i k \Omega_{0} \alpha\right)^{3}\left(r_{1}^{2}-r_{c}^{2}\right)^{2} r_{1}^{4}}\right)
\end{aligned}
$$

where $\Phi_{\infty}(r)$ is the steady-state solution (4.59).

Case 2, $\sqrt{2} r_{1}<r<\sqrt{2} r_{c}$

For $\sqrt{2} r_{1}<r<\sqrt{2} r_{c}, r \neq r_{c}$, the configuration for the inversion of the Laplace transform in the complex $s$-plane is shown in Figure 5.1b. The pole is in region I. There is only one branch point and the solution takes the form

$$
\begin{aligned}
& \phi(r, t) \sim \Phi_{\infty}(r) e^{-i \omega t} \\
& +e^{-i \omega t} e^{i k \Omega_{0} \alpha\left(r^{2}-r_{c}^{2}\right) t}\left(\frac{r}{r_{1}}\right)^{-k}\left(\frac{2 h_{1}(r)}{t^{2}\left(i k \Omega_{0} \alpha\right)^{2}\left(r^{2}-r_{c}^{2}\right) r^{2}}+\frac{2 h_{2}(r)}{t^{3}\left(i k \Omega_{0} \alpha\right)^{3}\left(r^{2}-r_{c}^{2}\right)^{2} r^{4}}\right)
\end{aligned}
$$

where $\Phi_{\infty}(r)$ is the steady-state solution (4.59). 
Case 3, $r>\sqrt{2} r_{c}$

For $r>\sqrt{2} r_{c}$, the configuration for the inversion of the Laplace transform in the complex $s$-plane is shown in Figure 5.2. The pole is in region II. There is only one branch point and the solution is

$$
\begin{aligned}
& \phi(r, t) \sim \Phi_{\infty}(r) e^{-i \omega t} \\
& +e^{-i \omega t} e^{i k \Omega_{0} \alpha\left(r^{2}-r_{c}^{2}\right) t}\left(\frac{r}{r_{1}}\right)^{-k}\left(\frac{2 h_{1}(r)}{t^{2}\left(i k \Omega_{0} \alpha\right)^{2}\left(r^{2}-r_{c}^{2}\right) r^{2}}+\frac{2 h_{2}(r)}{t^{3}\left(i k \Omega_{0} \alpha\right)^{3}\left(r^{2}-r_{c}^{2}\right)^{2} r^{4}}\right),
\end{aligned}
$$

where $\Phi_{\infty}(r)$ is the steady-state solution of the form (4.60).

We observe that each of these solutions (5.75)-(5.77) comprises a term $\Phi_{\infty}(r) e^{-i \omega t}$, where $\Phi_{\infty}(r)$ is the corresponding steady-amplitude solution from chapter 4 , and one or two time-dependent terms with frequencies $\omega_{1}=\omega-k \Omega_{0} \alpha\left(r^{2}-r_{c}^{2}\right)=k \Omega_{0} \alpha r^{2}$ and $\omega_{2}=\omega-k \Omega_{0} \alpha\left(r_{1}^{2}-r_{c}^{2}\right)=k \Omega_{0} \alpha r_{1}^{2}$.

The solutions (5.75), (5.76) and (5.77) give a complete description of the solution of the linear time-dependent problem (5.1) for $r_{1}<r<1 / \sqrt{\alpha}, r \neq r_{c}$, the interval shown in Figure 4.2. The solution (5.76) is valid around the critical radius $r=r_{c}$, but not at $r=r_{c}$. In order to find a solution at $r=r_{c}$, we need to consider an inner region, defined by a critical layer around the point $r=r_{c}$.

In deriving the solution (5.76) we considered $\frac{\rho}{\left|r-r_{c}\right|}<1$ in order to make the approximation (5.52). Integrating the geometric series (5.52) term by term gave us series of terms proportional to $\left[\left(r-r_{c}\right) t\right]^{-n}$ which is convergent for $\left|r-r_{c}\right| t>1$. Thus, the solution (5.76) is valid for $t \gg 1$ and $\left|r-r_{c}\right| t>1$, outside of a layer of thickness $\frac{1}{t}$ centred at $r=r_{c}$. On the edge of this layer where $\left(r-r_{c}\right) t \sim O(1)$ and inside the layer where $\left|r-r_{c}\right| t<1$, the solution (5.76) is not valid. The region $\left|r-r_{c}\right|>\frac{1}{t}$ is 
considered to be the outer region.

In the inner layer where $\left|r-r_{c}\right| t \sim O(1)$ we can not use this solution because of the singularity $\frac{1}{r-r_{c}}$. The solution in the inner layer will be considered in the next section.

\subsection{Time-dependent solution close to the critical layer}

To find the solution at the edge of the critical layer where $\left|r-r_{c}\right| t \sim O(1)$, we return to the evaluation of the integral (5.58). In the outer region we considered $\frac{\rho}{\left|r-r_{c}\right|} \ll 1$ and approximated $\frac{1}{s+i \omega}$ by

$$
\frac{1}{s+i \omega} \sim \frac{1}{r-r_{c}} \sum_{n=1}^{\infty} \int_{0}^{\infty} c_{n}\left(\frac{\rho}{r-r_{c}}\right)^{n} e^{-\rho t} d \rho, \frac{\rho}{\left|r-r_{c}\right|}<1
$$

Close to the critical layer $\left|r-r_{c}\right| t \sim O(1)$ and $\frac{\rho}{r-r_{c}} \sim O(1)$, so we need to retain all the terms in the expansion (5.78).

We also expand $\bar{\Omega}(r)=\Omega_{0}\left(1-\alpha r^{2}\right)$ in powers of $r-r_{c}$. Since $\bar{\Omega}(r)$ is analytic at $r_{c}$, we can use its Taylor series expansion

$$
\bar{\Omega}(r) \sim \bar{\Omega}\left(r_{c}\right)+\bar{\Omega}^{\prime}\left(r_{c}\right)\left(r-r_{c}\right)+O\left(\left(r-r_{c}\right)^{2}\right) \sim \frac{\omega}{k}-2 \alpha \Omega_{0} r_{c}\left(r-r_{c}\right)+O\left(\left(r-r_{c}\right)^{2}\right) .
$$

Recall, that $\omega / k=\bar{\Omega}\left(r_{c}\right)$. 
Now we can write (5.58) in the form

$$
\begin{aligned}
&\left(\int_{C_{4}}+\int_{C_{5}}\right) \tilde{\phi}(r, s) e^{s t} d s \\
& \sim e^{-i \omega t} e^{i k \Omega_{0} \alpha\left(r^{2}-r_{c}^{2}\right) t}\left(\frac{r}{r_{1}}\right)^{-k}(2 i \pi) \int_{0}^{\infty} \sum_{n=0}^{\infty}\left(\frac{\rho e^{ \pm i \pi}}{i k \Omega_{0} \alpha 2 r_{c}\left(r-r_{c}\right)}\right)^{n} \\
&\left(\frac{2 \rho h_{1}(r)}{\left(i k \Omega_{0} \alpha\right)^{2} r^{2} 2 r_{c}\left(r-r_{c}\right)}+\frac{\rho^{2} h_{4}(r)}{\left(i k \Omega_{0} \alpha\right)^{3} r^{4} 2 r_{c}\left(r-r_{c}\right)}\right) e^{-\rho t} d \rho, \\
& \rho \ll 1 .
\end{aligned}
$$

where

$$
h_{4}(r)=h_{1}(r)-4 h_{1}^{2}(r)\left(i \Omega_{0} \alpha\right)^{2} r_{1}^{2} 2 r_{c}\left(r-r_{c}\right)\left(\log \frac{r^{2}-r_{1}^{2}}{r^{2}}+1\right)
$$

So

$$
\begin{aligned}
\left(\int_{C_{4}}+\right. & \left.\int_{C_{5}}\right) \tilde{\phi}(r, s) e^{s t} d s \sim e^{-i \omega t} e^{-2 i k \Omega_{0} \alpha r_{c}\left(r_{c}-r\right) t}\left(\frac{r}{r_{1}}\right)^{-k}(2 i \pi) \\
& \int_{0}^{\infty}\left(\frac{2 h_{1}}{\left(i k \Omega_{0} \alpha\right)^{2} r^{2}\left(r_{c}-r\right)} \sum_{n=0}^{\infty} \frac{(\rho)^{n+1}}{\left(i k \Omega_{0} \alpha 2 r_{c}\left(r_{c}-r\right)\right)^{n}}\right. \\
& \left.-\frac{2 h_{4}}{\left(i k \Omega_{0} \alpha\right)^{3} r^{4} 2 r_{c}\left(r_{c}-r\right)} \sum_{n=0}^{\infty} \frac{(\rho)^{n+2}}{\left(i k \Omega_{0} \alpha 2 r_{c}\left(r_{c}-r\right)\right)^{n}}\right) e^{-\rho t} d \rho, \rho \ll 1 .
\end{aligned}
$$

Again making use of (5.62), we can write (5.82) in terms of the gamma function as

$$
\begin{aligned}
&\left(\int_{C_{4}}+\int_{C_{5}}\right) \tilde{\phi}(r, s) e^{s t} d s \sim e^{-i \omega t} e^{-2 i k \Omega_{0} \alpha r_{c}\left(r_{c}-r\right) t}\left(\frac{r}{r_{1}}\right)^{-k} \frac{1}{\left(i k \Omega_{0} \alpha r^{2}\right)}(2 i \pi) \\
&\left(\frac{2 h_{1}}{i k \Omega_{0} \alpha r^{2}} \sum_{n=0}^{\infty} \frac{\Gamma(n+2)}{\left(2 i k \Omega_{0} \alpha r_{c}\right)^{n+1}\left(r_{c}-r\right)^{n+1} t^{n+2}}\right. \\
&\left.+\frac{2 h_{4}(r)}{\left(i k \Omega_{0} \alpha\right)^{2} r^{4}} \sum_{n=0}^{\infty} \frac{\Gamma(n+3)}{\left(2 i k \Omega_{0} \alpha r_{c}\right)^{n+1}\left(r_{c}-r\right)^{n+1} t^{n+3}}\right), t \gg 1
\end{aligned}
$$


Close to the critical layer where $\left|r-r_{c}\right| t \sim O(1)$, the solution is

$$
\begin{aligned}
\left(\int_{C_{4}}+\right. & \left.\int_{C_{5}}\right) \tilde{\phi}(r, s) e^{s t} d s \sim e^{-i \omega t} e^{-2 i k \Omega_{0} \alpha r_{c}\left(r_{c}-r\right) t}\left(\frac{r}{r_{1}}\right)^{-k} \frac{2 i \pi}{\left(i k \Omega_{0} \alpha r^{2}\right)} \\
& \left(\frac{2 h_{1}(r)}{i k \Omega_{0} \alpha r^{2} t} \sum_{n=0}^{\infty} \frac{\Gamma(n+2)}{\left(2 i k \Omega_{0} \alpha r_{c}\right)^{n+1}\left(r_{c}-r\right)^{n+1} t^{n+1}}\right. \\
& \left.-\frac{2 h_{4}(r)}{\left(i k \Omega_{0} \alpha\right)^{2} r^{4} t^{2}} \sum_{n=0}^{\infty} \frac{\Gamma(n+3)}{\left(2 i k \Omega_{0} \alpha r_{c}\right)^{n+1}\left(r_{c}-r\right)^{n+1} t^{n+1}}\right), t \gg 1
\end{aligned}
$$

Note that all the terms in both series are of the same order in the critical layer where $\left|r-r_{c}\right| t \sim O(1)$. Following Campbell and Maslowe (1998), the series in (5.84) can be expressed in terms of exponential integrals which are defined as

$$
E_{k}^{+}(z)=\int_{1}^{\infty} \frac{e^{-z u}}{u^{k}} d u, \operatorname{Re}(z) \geq 0
$$

and

$$
E_{k}^{-}(z)=\int_{1}^{\infty} \frac{e^{z u}}{u^{k}} d u, \operatorname{Re}(z) \geq 0
$$

After integrating by parts we get

$$
E_{k}^{ \pm}(z)=\frac{e^{\mp z}}{\Gamma(k)} \sum_{n=0}^{n=\infty} \frac{\Gamma(n+k)}{( \pm z)^{n+1}}
$$

So close to the critical layer where $\left(r-r_{c}\right) t \sim O(1)$, the solution is

$$
\begin{aligned}
\phi(r, t) & \sim \Phi_{\infty}(r) e^{-i \omega t}-e^{-i \omega t}\left(\frac{r}{r_{1}}\right)^{-k}\left(\frac{h_{1}(r)}{i k \Omega_{0} \alpha r^{2} t} E_{2}^{s g n\left(r-r_{c}\right)}\left(2 i k r_{c}^{2} \alpha\left|r_{c}-r\right| t\right)\right. \\
& \left.-\frac{2 h_{4}(r)}{\left(i k \Omega_{0} \alpha r^{2}\right)^{2} t^{2}} E_{3}^{s g n\left(r-r_{c}\right)}\left(2 i k r_{c}^{2} \alpha\left|r_{c}-r\right| t\right)+O\left(\frac{1}{t^{3}}\right)\right)
\end{aligned}
$$

where $h_{1}$ and $h_{4}$ are defined by (5.60) and (5.81) and $\Phi_{\infty}(r)$ is the steady-state solution which was derived in chapter 4 . Close to the critical layer, the solution $\Phi_{\infty}(r)$ is in the 
form (4.59). The solution (5.88) consists of the steady part and terms proportional to negative powers of $t$. As $t \rightarrow \infty$ the solution converges to the steady state solution.

So, we have found the solution (5.76) valid for $\left|r-r_{c}\right| t \gg 1, t \gg 1$, and the solution (5.88) valid for $\left|r-r_{c}\right| t \sim O(1), t \gg 1$.

\subsection{The inner layer solution}

The time-dependent solution (5.76) and its derivatives with respect to $r$ are singular at $r=r_{c}$. The steady term $\Phi_{\infty}$ includes a term proportional to $\left(1-\frac{r^{2}}{r_{c}^{2}}\right) \log \left(1-\frac{r^{2}}{r_{\mathrm{c}}^{2}}\right)$ which is singular at $r=r_{c}$ and the time-dependent terms of solution (5.76) include terms proportional to $\frac{1}{r-r_{c}}$. In order to find a solution that is nonsingular at $r=r_{c}$ we have to consider a so-called inner layer around $r=r_{c}$.

We can write the solution (5.88) for $r$ close to $r_{c}$ in terms of $\left(r-r_{c}\right)$ as

$$
\begin{aligned}
\phi(r, t) & \sim \Phi_{\infty}(r) e^{-i \omega t}-e^{-i \omega t}\left(\frac{r}{r_{1}}\right)^{-k}\left(\frac{h_{1}(r)}{i k \Omega_{0} \alpha r^{2} t} E_{2}^{s g n\left(r-r_{c}\right)}\left(2 i k r_{c}^{2} \alpha\left|r_{c}-r\right| t\right)\right. \\
& \left.-\frac{2 h_{4}(r)}{\left(i k \Omega_{0} \alpha r^{2}\right)^{2} t^{2}} E_{3}^{s g n\left(r-r_{c}\right)}\left(2 i k r_{c}^{2} \alpha\left|r_{c}-r\right| t\right)+O\left(\frac{1}{t^{3}}\right)\right)
\end{aligned}
$$

where $\Phi_{\infty}(r)$ is defined by (4.59). The leading-order terms of $\Phi_{\infty}(r)$ also can be written in terms of $\left(r-r_{c}\right)$ as

$$
\begin{aligned}
\Phi_{\infty}(r) \sim e^{-i \omega t} h_{1}\left(r_{c}\right)\left(\frac{r}{r_{c}}\right)^{-k}\left[1-\frac{4}{r_{c}^{2}}\left(r_{c}-r\right) \log \left(r_{c}-r\right)\right. \\
\left.-\frac{4}{r_{c}^{2}}\left(r_{c}-r\right) \log \left(\frac{2}{r_{c}}\right)+O\left(r_{c}-r\right)^{2}\right],\left|r-r_{c}\right|<1,
\end{aligned}
$$

where

$$
h_{1}\left(r_{c}\right)=\left(1-2\left(1-\frac{r_{1}^{2}}{r_{c}^{2}}\right) \log \left(1-\frac{r_{1}^{2}}{r_{c}^{2}}\right)\right)^{-1}
$$

We wish to find a nonsingular solution valid in the inner region, where $\left|r-r_{c}\right|<\frac{1}{t}$ 
for $t \gg 1$. On a time scale of $t \sim O\left(\mu^{-1}\right)$, where $\mu \ll 1$, the thickness of the critical layer at time $t$ is $\left|r-r_{c}\right| \sim O(\mu)$. So we define a new radial variable

$$
Z=\frac{1}{\mu}\left(r-r_{c}\right)
$$

and a slow-time variable

$$
T=\mu t
$$

At the edge of the critical layer $|Z| T \sim O(1)$, and inside the critical layer $|Z| T<O(1)$.

We rewrite (5.88) in terms of $Z, T$ and $\mu$ as

$$
\begin{array}{r}
\phi(Z, T, t)=e^{-i \omega t} h_{1}(r)\left(1+\mu \log \mu\left(\frac{4}{r_{c}}\right) Z+\mu\left(\left(\frac{4}{r_{c}} \log \frac{2}{r_{c}}\right) Z+\frac{4}{r_{c}} Z \log (-Z)\right)\right) \\
-\mu \frac{2 h_{1}(r)}{i k \Omega_{0} \alpha r^{2} T} E_{2}\left(i k \Omega_{0} 2 \alpha r_{c}|Z| T\right)+O\left(\mu^{2}\right) .(5
\end{array}
$$

Making use of the following property of the exponential integral $E_{2}$ (Abramowitz and Stegun, 1964, equation 5.1.12) that

$$
E_{2}^{ \pm}(z) \sim \pm\left(z \log ( \pm z)+\left(\gamma_{E}-1\right) z\right)-\sum_{n=0, n \neq 1}^{\infty} \frac{(-z)^{n}}{(n-1) n !}
$$

where $\gamma_{E}$ is Euler's constant, we can rewrite (5.94) as

$$
\begin{array}{r}
\phi(Z, T, t) \sim\left(\frac{r}{r_{1}}\right)^{-k} e^{-i \omega t}\left(h_{1}\left(r_{c}\right)+\mu \log \mu\left(\frac{4 h_{1}}{r_{c}}\right) Z+\mu\left(\frac{4 h_{1}}{r_{c}} \log \frac{2}{r_{c}}\right) Z\right. \\
+\mu \frac{4 h_{1}}{r_{c}} Z \log (-Z)-\mu \frac{2 h_{1}\left(r_{c}\right)}{i k \Omega_{0} \alpha r_{c}^{2} T}\left(i k 2 \Omega_{0} \alpha r_{c} Z T\right) \log \left(-i k 2 \Omega_{0} \alpha r_{c} Z T\right) \\
+\mu \frac{2 h_{1}\left(r_{c}\right)}{i k \Omega_{0} \alpha r_{c}^{2} T}\left(\gamma_{E}-1\right)\left(i k 2 \Omega_{0} \alpha r_{c} Z T\right)+ \\
\left.\mu \frac{2 h_{1}\left(r_{c}\right)}{i k \Omega_{0} \alpha r_{c}^{2} T} \sum_{n=0, n \neq 1}^{\infty} \frac{\left(-2 i k \Omega_{0} \alpha r_{c} Z T\right)}{(n-1) n !}+O\left(\mu^{2}\right)\right) .
\end{array}
$$


After some simplifications, (5.96) becomes

$$
\begin{array}{r}
\phi(Z, T, t) \sim e^{-i \omega t}\left(\frac{r}{r_{1}}\right)^{-k}\left[h_{1}\left(r_{c}\right)+\mu \log \mu\left(\frac{4 h_{1}}{r_{c}}\right) Z+\mu\left(\frac{4 h_{1}}{r_{c}} \log \frac{2}{r_{c}}\right) Z\right. \\
+\mu \frac{4 h_{1}}{r_{c}} Z \log (-Z)-\mu \frac{4 h_{1}\left(r_{c}\right)}{r_{c}} Z \log \left(2 i k \Omega_{0} \alpha r_{c} T\right)-\mu \frac{4 h_{1}\left(r_{c}\right)}{r_{c}} Z \log (-Z) \\
\left.-\mu \frac{4 h_{1}\left(r_{c}\right)}{r_{c}}\left(\gamma_{E}-1\right) Z+\mu \frac{2 h_{1}\left(r_{c}\right)}{i k \Omega_{0} \alpha r_{c}^{2} T} \sum_{n=0, n \neq 1}^{\infty} \frac{\left(-2 i k \Omega_{0} \alpha r_{c} Z T\right)^{n}}{(n-1) n !}+O\left(\mu^{2}\right)\right] \\
|Z T| \lesssim 1 .
\end{array}
$$

We see that the terms with $Z \log (-Z)$ cancel each other and so we obtain a nonsingular inner solution

$$
\begin{aligned}
\phi_{\text {inner }}(Z, T, t) & \sim e^{-i \omega t}\left(\frac{r}{r_{1}}\right)^{-k} e^{-i \omega t} h_{1}\left(r_{c}\right)\left(1+\mu \log \mu \frac{4}{r_{c}} Z+\mu\left(\frac{4}{r_{c}} \log \frac{2}{r_{c}}\right) Z\right. \\
& -\mu \frac{4}{r_{c}} Z \log \left(-2 i k \Omega_{0} \alpha r_{c} T\right)-\mu \frac{4}{r_{c}}\left(\gamma_{E}-1\right) Z \\
& \left.+\mu \frac{1}{T}\left[\frac{2}{i k \Omega_{0} \alpha r_{c}^{2}}+8 i k \Omega_{0} \alpha Z^{2} T^{2}+\frac{4 k^{2} \Omega_{0}^{2} \alpha^{2} r_{c} Z^{3} T^{3}}{3}+O\left(Z^{4} T^{4}\right)\right]\right) .
\end{aligned}
$$

In terms of the variables $\left(r-r_{c}\right)$ and $t,(5.98)$ can be written as

$$
\begin{aligned}
& \phi_{\text {inner }}(r, t) \sim e^{-i \omega t}\left(\frac{r}{r_{1}}\right)^{-k} e^{-i \omega t} h_{1}\left(r_{c}\right)\left(1+\log \mu \frac{-4}{r_{c}}\left(r_{c}-r\right)\right. \\
& -\left(\frac{4}{r_{c}} \log \frac{2}{r_{c}}\right)\left(r_{c}-r\right)+\frac{4}{r_{c}}\left(r_{c}-r\right) \log \left(-2 i k \mu \Omega_{0} \alpha r_{c} t\right)+\frac{4}{r_{c}}\left(\gamma_{E}-1\right)\left(r_{c}-r\right)+ \\
& \left.+\frac{1}{t}\left[\frac{2}{i k \Omega_{0} \alpha r_{c}^{2}}+8 i k \Omega_{0} \alpha\left(r_{c}-r\right)^{2} t^{2}+\frac{4 k^{2} \Omega_{0}^{2} \alpha^{2} r_{c}\left(r_{c}-r\right)^{3} t^{3}}{3}+O\left(\left(r_{c}-r\right)^{4} t^{4}\right)\right]\right)
\end{aligned}
$$

This solution is valid for $|Z| T \lesssim 1$, or $\left|\left(r-r_{c}\right)\right| t \lesssim 1$. In contrast with the outer region where the solution tends to a steady state as $t \rightarrow \infty$, the inner solution grows like $\log (t)$. From this we note that the qualitative behaviour of the solution for this linear configuration for vortex waves with $\beta=0$ is the same as for Rossby waves 
(with $\beta \neq 0$ ) in a configuration with rectangular geometry (Dickinson, 1970, Warn and Warn, 1976).

In the next chapter we introduce nonlinearity and the $\beta$-effect to the vortex wave problem.

\subsection{Discussion of the linear time-dependent solu- tion}

We discuss the linear time-dependent solution we have obtained here and compare with the conclusions made by other researchers who have examined similar configuration. The problem of wave-mean flow interactions at a critical layer has been studied by many previous researchers in the context of barotropic Rossby waves in a rectangular domain (Dickinson, 1970, Warn and Warn, 1976, Warn and Warn, 1978, Campbell and Maslowe, 1998, Campbell, 2004). We see some similarities between the solutions we have derived in this chapter and those obtained in these previous studies. In the rectangular configuration as well, the time-dependent solution comprises the steady solution and time-dependent terms which are arises from the branch points of the integrand in the inverse Laplace transform. One branch point arises from the effect of the mean flow, the other from the effect of the boundary condition. In both problems in the outer layer time-dependent terms decrease with time as $1 / t$, and the inner layer solution is valid for $\left|r-r_{c}\right| t<1$. We note that in the rectangular configuration these effects arise because of the variation of Coriolis force, in our problem they arise even in the $f$-plane configuration.

The problem of deriving analytical solutions for vortex wave propagation in cyclones was considered by other researchers (see, e.g., Montgomery and Kallenbach, 
1997 and Brunet and Montgomery, 2002). We can compare our results with the results obtained by Brunet and Montgomery (2002) whose configuration is the most closely related to ours. They solved the initial-value problem on an $f$-plane and used an angular velocity profile in the form of a quadratic function, similar to the profile we used. They solved the initial-value problem using a Hankel transform. Their solution for large $r$ in the notation of this thesis is written as

$$
\phi(r, t) \sim(1+i t)^{-\mu / 4-1}\left(-r^{2}(1+i t)\right)^{k / 2} M\left(\frac{k}{2}+\frac{\sqrt{k^{2}+8}}{2}, k+1,-r^{2}(1+i t)\right),
$$

where $M(a, b, z)$ is Kummer's function.

Let us compare this result with the solution we derived in this thesis. For negative values of the real part of $z$, as $|z| \rightarrow \infty$, Kummer's function has the approximation (Abramowitz and Stegun, 1964, equation 13.1.5)

$$
M(a, b, z) \sim \frac{\Gamma(b)}{\Gamma(b-a)}(-z)^{-a}\left[1+O\left(|z|^{-1}\right)\right]
$$

for $|z| \rightarrow \infty$, where $\operatorname{Re}(z)<0$. So the asymptotic behaviour of the solution for large values of $\left|r^{2}(1+i t)\right|$ is

$$
\phi(r, t) \sim O\left(\left[-r^{2}(1+i t)\right]^{k / 2}\left[-r^{2}(1+i t)\right]^{-\frac{k}{2}-\frac{\sqrt{k^{2}+8}}{2}}\right) \sim O\left(r^{-\sqrt{k^{2}+8}}[1+i t]^{-\frac{\sqrt{k^{2}+8}}{2}}\right)
$$

For large $t$ this solution is proportional to $O\left(r^{-\sqrt{k^{2}+8}}\right)$ and it has the same qualitative behaviour as the steady-state solution (4.56) we obtained for $r>r_{c}$ which is proportional to $r^{-\sqrt{k^{2}+8}}$.

To figure out the reason for the constant of 8 in this expression, we have to go 
back to the beginning of chapter 4 , to the equation we solved for the steady case

$$
\phi^{\prime \prime}+\frac{\phi^{\prime}}{r}-\frac{k^{2}}{r^{2}} \phi+\frac{\frac{k}{r} \frac{\partial}{\partial r}\left(\bar{v}^{\prime}+\bar{v} / r\right)}{\omega-k \bar{\Omega}} \phi=0
$$

The numerator in the last term of equation (5.103) is $\frac{k}{r} \frac{\partial}{\partial r}\left(\bar{v}^{\prime}+\bar{v} / r\right)$. This expression can be written in terms of the streamfunction of the mean flow $\bar{\psi}(r)$ as

$$
\frac{1}{r} \frac{\partial}{\partial r}\left(\bar{v}^{\prime}+\bar{v} / r\right)=\frac{1}{r} \frac{\partial}{\partial r}\left(\bar{\psi}_{r r}+\frac{1}{r} \bar{\psi}_{r}\right)=\frac{1}{r} \frac{\partial}{\partial r} \nabla^{2} \bar{\psi}
$$

which is proportional to the radial gradient of the vorticity of the mean flow. With our choice of the angular velocity profile $\bar{\Omega}=\Omega_{0}\left(1-\alpha r^{2}\right)$, we have $\bar{v}(r)=\Omega_{0}\left(r-\alpha r^{3}\right)$ and so the gradient of the mean flow vorticity $(5.103)$ is $-8 \Omega_{0} \alpha r$. This is the reason for the 8 that appears in the power of $r$ in the solution. We conclude that the rate of attenuation of the vortex wave amplitude depends on the wave number $k$ and the gradient of the vorticity of the mean flow. 


\section{Chapter 6}

\section{Effect of nonlinearity and $\beta$-effect}

\subsection{Weakly-nonlinear analysis}

The linear time-dependent solutions derived in chapter 5 can be considered as a first approximation to the solution of the nonlinear equation (3.53)

$$
\left(\frac{\partial}{\partial t}+\frac{\bar{v}}{r} \frac{\partial}{\partial \lambda}\right) \nabla^{2} \psi-\frac{1}{r} \psi_{\lambda} \frac{\partial}{\partial r}\left(\bar{v}_{r}+\frac{1}{r} \bar{v}\right)=-\frac{\varepsilon}{r}\left(\psi_{r} \nabla^{2} \psi_{\lambda}-\psi_{\lambda} \nabla^{2} \psi_{r}\right)
$$

To obtain a better approximation we can consider (3.53) and carry out a weaklynonlinear analysis for $\varepsilon \ll 1$. We can express the solution of $(6.1)$ in powers of $\varepsilon$

$$
\psi(r, \lambda, t) \sim \psi^{(0)}(r, \lambda, t)+\varepsilon \psi^{(1)}(r, \lambda, t)+O\left(\varepsilon^{2}\right)
$$

where $\psi^{(1)}(r, \lambda, t) \sim O(1)$. The leading-order term satisfies the boundary condition $\psi^{(0)}\left(r_{1}, \lambda, t\right)=e^{i k \lambda}+$ c.c. and can be thus written as

$$
\psi^{(0)}(r, \lambda, t)=\phi^{(0)}(r, t) e^{i k \lambda}+\text { c.c. }
$$


When the expansion (6.2) is substituted into the nonlinear equation (6.1), at $O(1)$ we obtain the linear equation (3.54) which tells us that $\psi^{(0)}(r, \lambda, t)$ is the linear solution (5.76) that was derived in chapter 5 .

At $O(\varepsilon)$ we obtain

$$
\begin{array}{r}
\left(\frac{\partial}{\partial t}+\frac{\bar{v}}{r} \frac{\partial}{\partial \lambda}\right) \nabla^{2} \psi^{(1)}-\frac{1}{r} \psi_{\lambda}^{(1)} \frac{\partial}{\partial r}\left(\bar{v}_{r}+\frac{1}{r} \bar{v}\right) \\
=-\frac{\varepsilon}{r}\left(\psi_{r}^{(0)} \nabla^{2} \psi_{\lambda}^{(0)}-\psi_{\lambda}^{(0)} \nabla^{2} \psi_{r}^{(0)}\right)
\end{array}
$$

with the boundary conditions $\psi^{(1)}\left(r_{1}, \lambda, t\right)=0$. Substituting (6.3) into (6.4) gives

$$
\begin{gathered}
\left(\frac{\partial}{\partial t}+\frac{\bar{v}}{r} \frac{\partial}{\partial \lambda}\right) \nabla^{2} \psi^{(1)}-\frac{1}{r} \psi_{\lambda}^{(1)} \frac{\partial}{\partial r}\left(\bar{v}_{r}+\frac{1}{r} \bar{v}\right) \\
=-\frac{i k}{r}\left(\left(\phi^{(0)} \phi_{r r r}^{*(0)}+\phi_{r}^{(0)} \phi_{r r}^{*(0)}+\frac{1}{r} \phi^{(0)} \phi_{r r}^{*(0)}-\phi^{*(0)} \phi_{r r r}^{(0)}+\phi_{r}^{*(0)} \phi_{r r}^{(0)}+\frac{1}{r} \phi^{*(0)} \phi_{r r}^{(0)}\right)\right. \\
-e^{2 i k \lambda}\left(\left(\phi_{r}^{(0)}\right)^{2}+\phi_{r}^{(0)} \phi_{r r}^{(0)}-\frac{1}{r} \phi^{(0)} \phi_{r r}^{(0)}-\frac{1}{r} \phi^{(0)} \phi_{r r r}^{(0)}\right) \\
\left.-e^{-2 i k \lambda}\left(\left(\phi_{r}^{*(0)}\right)^{2}+\phi_{r}^{*(0)} \phi_{r r}^{*(0)}-\frac{1}{r} \phi^{*(0)} \phi_{r r}^{*(0)}-\frac{1}{r} \phi^{*(0)} \phi_{r r r}^{*(0)}\right)\right) \\
=-\frac{i k}{r}\left(2 \operatorname{Im}\left(\frac{\partial}{\partial r}\left(r \phi^{(0)} \phi_{r r}^{*(0)}\right)\right)-e^{2 i k \lambda}\left(\left(\phi_{r}^{(0)}\right)^{2}+\phi_{r}^{(0)} \phi_{r r}^{(0)}-\frac{1}{r} \phi^{(0)} \phi_{r r}^{(0)}-\frac{1}{r} \phi^{(0)} \phi_{r r r}^{(0)}\right)\right. \\
\left.-e^{-2 i k \lambda}\left(\left(\phi_{r}^{*(0)}\right)^{2}+\phi_{r}^{*(0)} \phi_{r r}^{*(0)}-\frac{1}{r} \phi^{*(0)} \phi_{r r}^{*(0)}-\frac{1}{r} \phi^{*(0)} \phi_{r r r}^{(* 0)}\right)\right)
\end{gathered}
$$

This implies that $\psi^{(1)}$ takes the form

$$
\psi^{(1)}(r, \lambda, t)=\bar{\phi}^{(1)}(r, t)+\phi^{(1)}(r, t) e^{2 i k \lambda}+\text { c.c. }
$$

where $\bar{\phi}^{(1)}(r, t)$ and $\phi^{(1)}(r, t)$ are complex functions. The first term in (6.6) is the zerowavenumber component which arises from the wave-mean flow interactions. This term satisfies

$$
\frac{\partial}{\partial t} \nabla^{2} \bar{\phi}^{(1)}=\frac{2 i k}{r} \operatorname{Im}\left(\frac{\partial}{\partial r}\left(r \phi^{(0)} \phi_{r r}^{*(0)}\right)\right)
$$


The evolution of this component is discussed in more detail in section 6.4. The second term in (6.6) and its complex conjugate are the leading-order contributions to the second harmonic. The function $\phi^{(1)}(r, t)$ satisfies

$$
\begin{aligned}
& \left(\frac{\partial}{\partial t}+\frac{\bar{v}}{r} \frac{\partial}{\partial \lambda}\right) \nabla^{2} \phi^{(1)}-\frac{1}{r} \phi_{\lambda}^{(1)} \frac{\partial}{\partial r}\left(\bar{v}_{r}+\frac{1}{r} \bar{v}\right) \\
& \quad=-\frac{i k}{r^{2}}\left(\left(\phi_{r}^{(0)}\right)^{2}-\phi^{(0)} \phi_{r r}^{(0)}+r \phi_{r}^{(0)} \phi_{r r}^{(0)}-r \phi^{(0)} \phi_{r r r}^{(0)}\right)
\end{aligned}
$$

In the outer region where $\left|r-r_{c}\right| t \gg 1$, for $t \gg 1$, the linear solution $\phi^{(0)}(r, t)$ is $(5.76)$

$$
\begin{aligned}
\phi^{(0)}(r, t) & \sim \Phi_{\infty}(r) e^{-i \omega t} \\
+ & e^{i k \Omega_{0} \alpha r^{2} t}\left(\frac{r}{r_{1}}\right)^{-k}\left(\frac{2 h_{1}(r)}{\left.t^{2}\left(i k \Omega_{0} \alpha\right)^{2}\right)\left(r^{2}-r_{c}^{2}\right) r^{2}}+\frac{2 h_{2}(r)}{t^{3}\left(i k \Omega_{0} \alpha\right)^{3}\left(r^{2}-r_{c}^{2}\right)^{2} r^{4}}\right) \\
+ & e^{i k \Omega_{0} \alpha r_{1}^{2} t}\left(\frac{r}{r_{1}}\right)^{-k}\left(\frac{h_{3}(r)}{\left.t^{2}\left(i k \Omega_{0} \alpha\right)^{2}\right)\left(r_{1}^{2}-r_{c}^{2}\right) r_{1}^{2}}+\frac{h_{2}(r)}{t^{3}\left(i k \Omega_{0} \alpha\right)^{3}\left(r_{1}^{2}-r_{c}^{2}\right)^{2} r_{1}^{4}}\right)
\end{aligned}
$$

where $\Phi_{\infty}(r)$ is the steady-state solution. In the inner region where $\left|r-r_{c}\right| t \ll 1$, the linear solution is

$$
\begin{aligned}
& \phi^{(0)}(r, t)=\phi_{\text {inner }}(r, t) \sim\left(\frac{r}{r_{1}}\right)^{-k} e^{-i \omega t} h_{1}\left(r_{c}\right)\left(1+\left(\frac{4}{r_{c}} \log \frac{2}{r_{c}}\right)\left(r-r_{c}\right)\right. \\
& -\frac{4}{r_{c}}\left(r-r_{c}\right) \log \left(-2 i k \Omega_{0} \alpha r_{c} t\right)-\frac{4}{r_{c}}\left(\gamma_{E}-1\right)\left(r-r_{c}\right) \\
& \left.+\frac{1}{t}\left[\frac{2}{i k \Omega_{0} \alpha r_{c}^{2}}+8 i k \Omega_{0} \alpha\left(r-r_{c}\right)^{2} t^{2}-\frac{4 k^{2} \Omega_{0}^{2} \alpha^{2} r_{c}\left(r-r_{c}\right)^{3} t^{3}}{3}+O\left(\left(r-r_{c}\right)^{4} t^{4}\right)\right]\right)
\end{aligned}
$$

The nonlinear terms on the right-hand side of (6.5) contain derivatives with respect to $r$. In the outer region where $\phi^{(0)}(r, t)$ is given by (6.9), we observe that each time that $\phi^{(0)}(r, t)$ is differentiated with respect to $r$, the terms with the factor of $e^{i k \Omega_{0} \alpha r^{2} t}$ are multiplied by a factor of $t$. This means that for $t \gg 1$, the leading-order terms on 
the right-hand side of (6.5) are proportional to

$$
t^{3} \frac{e^{i k \Omega_{0} \alpha\left(r^{2}-r_{c}^{2}\right)}}{t} \sim O\left(t^{2}\right)
$$

In the inner layer where $\phi^{(0)}(r, t)$ is given by $(6.10)$, we observe that $\phi^{(0)}(r, t)$ is $O(1)+O\left(r-r_{c}\right)+O(\log t)\left(r-r_{c}\right)(5.99)$ and so the first derivative of $\phi^{(0)}$ with respect to $r$ is $O(1)+O(\log t)$. We can write it in the form

$$
\begin{aligned}
\phi_{r}^{(0)}(r, t) \sim \frac{4 h_{1}\left(r_{c}\right)}{r_{c}} & \left(\log \frac{2}{r_{c}}-1+\frac{1}{i k \Omega_{0} 2 \alpha r_{c}\left(r-r_{c}\right) t} e^{i k \Omega_{0} 2 \alpha r_{c}\left(r-r_{c}\right) t}\right. \\
+ & \log \left(-i k \Omega_{0} 2 \alpha r_{c} t\right)+\left(1-\gamma_{E}\right) \frac{1}{i k \Omega_{0} 2 \alpha r_{c}\left(r-r_{c}\right) t} \\
& \left.+\sum_{n=1}^{\infty} \frac{\left(-i k \Omega_{0} 2 \alpha r_{c}\left(r-r_{c}\right) t\right)^{n}}{n(n+1) !}\right),\left|r-r_{c}\right| t<1
\end{aligned}
$$

In the first derivative (6.11) there is the term proportional to $e^{i k \Omega_{o} \alpha 2 r_{c}\left(r-r_{c}\right) t}$. When $\phi_{r}^{(0)}$ is differentiated with respect to $r$, it is multiplied by a factor of $t$. So, the second derivative with respect to $r$ is $O(t)$, and the third derivative is $O\left(t^{2}\right)$.

Thus, for large $t$, the terms on the right-hand side of equation (6.1) are $O\left(t^{2}\right)$. For large $t$ the nonlinear terms increase as $O\left(t^{2}\right)$ and become $O(1)$ on a time-scale of $t \sim O\left(\varepsilon^{-1 / 2}\right)$. We can conclude that the nonlinear expansion (6.3) is valid for $t<O\left(\varepsilon^{-1 / 2}\right)$. To examine the late-time nonlinear solution we would have to define a slow-time variable $\tau=\varepsilon^{1 / 2} t$.

All the solutions derived up to this point are for vortex wave propagation on an $f$-plane where the Coriolis parameter is assumed to be a nonzero constant. In the next section we reintroduce the $\beta$-effect into the governing equations and examine first a linear and then, in section 6.3, the nonlinear configuration. 


\subsection{Effect of the variation of the Coriolis force}

The vortex Rossby wave equation with the $\beta$-effect included is (3.52)

$$
\begin{array}{r}
\left(\frac{\partial}{\partial t}+\frac{\bar{v}}{r} \frac{\partial}{\partial \lambda}\right) \nabla^{2} \psi-\frac{1}{r} \psi_{\lambda} \frac{\partial}{\partial r}\left(\bar{v}_{r}+\frac{1}{r} \bar{v}\right)-\frac{\beta}{r} \psi_{\lambda} \sin \lambda+\frac{\beta}{\varepsilon} \bar{v} \cos \lambda+\beta \psi_{r} \cos \lambda= \\
-\frac{\varepsilon}{r}\left(\psi_{r} \nabla^{2} \psi_{\lambda}-\psi_{\lambda} \nabla^{2} \psi_{r}\right) .
\end{array}
$$

The problem is governed by two nondimensional parameters $\beta$ and $\varepsilon$. In section 3.1 we demonstrated that $\beta$ can be considered as a small parameter given the typical length scale for our problem, which gives $\beta \sim 10^{-2}$. In general, wave amplitudes are small relative to the magnitude of the mean flow so the parameter $\varepsilon$ can also be considered to be small. That justifies the use of weakly-nonlinear analyses such as the analysis that was carried out in section 6.1. The first step in our investigation of the $\beta$-effect shall be to examine the linear problem with the $\beta$-effect added. This corresponds to a configuration where $\beta \gg \varepsilon$ and so the nonlinear $O(\varepsilon)$ terms in (6.12) appear at higher order than the $O(\beta)$ terms. However this scaling means that the $O\left(\beta \varepsilon^{-1}\right)$ term in $(6.12)$ becomes the dominant term in the equation. Since this term cannot satisfy the specified $O(1)$ boundary conditions, the linear problem is not welldefined in that case. In order to overcome this issue we would have to neglect the

$O\left(\beta \varepsilon^{-1}\right)$ term. We can do this by adding an additional term $\tilde{B}(r, \lambda)=-\frac{\beta}{\varepsilon} \bar{v} \cos \lambda$ to the governing equation as discussed in section 3.1. In section 6.3 we consider the full nonlinear equation (6.12) with both parameters $\varepsilon$ and $\beta$ included. We shall assume there that $\beta \ll \varepsilon$ which is the more likely physical configuration.

The linear equation with the addition of the term $\tilde{B}$ becomes

$$
\left(\frac{\partial}{\partial t}+\frac{\bar{v}}{r} \frac{\partial}{\partial \lambda}\right) \nabla^{2} \psi-\frac{1}{r} \psi_{\lambda} \frac{\partial}{\partial r}\left(\bar{v}_{r}+\frac{1}{r} \bar{v}\right)-\frac{\beta}{r} \psi_{\lambda} \sin \lambda+\beta \psi_{r} \cos \lambda=0
$$


To solve (6.13) we look for a solution of the form

$$
\psi(r, \lambda, t)=\psi^{(0)}(r, \lambda, t)+\beta \psi^{(1)}(r, \lambda, t)+O\left(\beta^{2}\right)
$$

where $\psi^{(0)}(r, \lambda, t)$ is the linear solution of (3.54) obtained in chapter 5 for the $f$-plane configuration. As in section 6.1 , we write $\psi^{(0)}(r, \lambda, t)$ as

$$
\psi^{(0)}(r, \lambda, t)=\phi^{(0)}(r, t) e^{i k \lambda}+\text { c.c. }
$$

After substitution of (6.14) into the governing equation (6.13) we obtain at $O(\beta)$ :

$$
\left(\frac{\partial}{\partial t}+\frac{\bar{v}}{r} \frac{\partial}{\partial \lambda}\right) \nabla^{2} \psi^{(1)}-\frac{1}{r} \psi_{\lambda}^{(1)} \frac{\partial}{\partial r}\left(\bar{v}_{r}+\frac{1}{r} \bar{v}\right)=\frac{\beta}{r} \psi_{\lambda}^{(0)} \sin \lambda-\beta \psi_{r}^{(0)} \cos \lambda
$$

with the boundary conditions $\psi^{(1)}(r, \lambda, t)=0$. Writing $\cos \lambda=\left(e^{i \lambda}+e^{-i \lambda}\right) / 2$ and $\sin \lambda=\left(e^{i \lambda}-e^{-i \lambda}\right) / 2 i$ and substituting (6.15) into (6.16), we obtain

$$
\begin{aligned}
& \left(\frac{\partial}{\partial t}+\frac{\bar{v}}{r} \frac{\partial}{\partial \lambda}\right) \nabla^{2} \psi^{(1)}-\frac{1}{r} \psi_{\lambda}^{(1)} \frac{\partial}{\partial r}\left(\bar{v}_{r}+\frac{1}{r} \bar{v}\right) \\
& \quad=\frac{1}{2}\left[\left(\frac{k}{r} \phi^{(0)}-\phi_{r}^{(0)}\right) e^{i(k+1) \lambda}-\left(\frac{k}{r} \phi^{(0)}+\phi_{r}^{(0)}(r)\right) e^{i(k-1) \lambda}\right]+\text { c.c. }
\end{aligned}
$$

This tells us that $\psi^{(1)}(r, \lambda, t)$ can be written as

$$
\psi^{(1)}(r, \lambda, t)=\psi^{(1 a)}(r, \lambda, t)+\psi^{(1 b)}(r, \lambda, t)
$$

where $\psi^{(1 a)}$ satisfies

$$
\begin{array}{r}
\left(\frac{\partial}{\partial t}+\frac{\bar{v}}{r} \frac{\partial}{\partial \lambda}\right) \nabla^{2} \psi^{(1 a)}-\frac{1}{r} \psi_{\lambda}^{(1 a)} \frac{\partial}{\partial r}\left(\bar{v}_{r}+\frac{1}{r} \bar{v}\right) \\
=\frac{1}{2}\left(\frac{k}{r} \phi^{(0)}-\phi_{r}^{(0)}\right) e^{i(k+1) \lambda}+\text { c.c. }
\end{array}
$$


and $\psi^{(1 b)}$ satisfies

$$
\begin{array}{r}
\left(\frac{\partial}{\partial t}+\frac{\bar{v}}{r} \frac{\partial}{\partial \lambda}\right) \nabla^{2} \psi^{(1 b)}-\frac{1}{r} \psi_{\lambda}^{(1 b)} \frac{\partial}{\partial r}\left(\bar{v}_{r}+\frac{1}{r} \bar{v}\right) \\
=-\frac{1}{2}\left(\frac{k}{r} \phi^{(0)}+\phi_{r}^{(0)}(r)\right) e^{i(k-1) \lambda}+\text { c.c. }
\end{array}
$$

with boundary conditions $\psi^{(1 a)}\left(r_{1}, \lambda, t\right)=0$ and $\psi^{(1 b)}\left(r_{1}, \lambda, t\right)=0$.

Each of the equations (6.19) and (6.20) is a nonhomogeneous differential equation, so we can write the general solution in each case as the sum of the general solution of the corresponding homogeneous equation and a particular solution of the nonhomogeneous equation, which is proportional to $e^{i(k \pm 1) \lambda}+$ c.c. Recall that the function $\phi^{(0)}(r, t)$ is the sum of $\Phi_{\infty}(r) e^{-i \omega t}$ and two time-dependent terms that go to zero as $t \rightarrow \infty$. Therefore, a particular solution of each of (6.19) and (6.20) is proportional to $e^{i((k \pm 1) \lambda-\omega t)}$ for $t \gg 1$. The zero boundary condition at $r=r_{1}$ tells us that the solution of the homogeneous equation is also proportional to $e^{i((k \pm 1) \lambda-\omega t)}$ for $t \gg 1$. Thus the solutions of the homogeneous equations corresponding to each of (6.19) and (6.20) are of the same form as the solution $\phi^{(0)}$, but with the wavenumber $k$ replaced by $k \pm 1$. For $t \gg 1$ the solution of the homogeneous equations at leading order are

$$
\psi_{\text {hom }}^{(1 a)}(r, \lambda, t)=\tilde{\phi}^{(1 a)}(r) e^{i((k+1) \lambda-\omega t)},
$$

and

$$
\psi_{h o m}^{(1 b)}(r, \lambda, t)=\tilde{\phi}^{(1 b)}(r) e^{i((k-1) \lambda-\omega t)}
$$

where $\tilde{\phi}^{(1 a)}$ and $\tilde{\phi}^{(1 b)}$ satisfy equations

$$
\tilde{\phi}^{(1 a)^{\prime \prime}}+\frac{\tilde{\phi}^{(1 a)^{\prime}}}{r}-\frac{(k+1)^{2}}{r^{2}} \tilde{\phi}^{(1 a)}-\frac{8 \Omega_{0} \alpha}{\left(\Omega_{0}\left(1-\alpha r^{2}\right)(k+1)-\omega\right)} \tilde{\phi}^{(1 a)}=0
$$


and

$$
\tilde{\phi}^{(1 b)^{\prime \prime}}+\frac{\tilde{\phi}^{(1 b)^{\prime}}}{r}-\frac{(k-1)^{2}}{r^{2}} \tilde{\phi}^{(1 b)}-\frac{8 \Omega_{0} \alpha}{\left(\Omega_{0}\left(1-\alpha r^{2}\right)(k-1)-\omega\right)} \tilde{\phi}^{(1 b)}=0
$$

Equation (6.23) has a singular point at the value of $r$ where $\omega=(k+1) \Omega_{0}\left(1-\alpha r^{2}\right)$, i.e. the mode with wavenumber $k+1$ has a critical radius at

$$
r_{c_{k+1}}=\frac{1}{\alpha^{1 / 2}}\left(1-\frac{\omega}{(k+1) \Omega_{0}}\right)^{1 / 2}
$$

Similarly, the mode with wavenumber $(k-1)$ from $(6.24)$ has a critical radius at

$$
r_{c_{k-1}}=\frac{1}{\alpha^{1 / 2}}\left(1-\frac{\omega}{(k-1) \Omega_{0}}\right)^{1 / 2}
$$

The wavenumber $k$ is the number of wavelengths in a full circle $0 \leq \lambda<2 \pi$, so $k$ is a positive integer. We can consider two cases $k=1$ and $k>1$. If $k=1$, then $k+1=2$ and $k-1=0$. This means that a zerowavenumber (non-oscillatory) component is generated by the $\beta$-effect. This component represents a change in the mean flow and will be discussed in section 6.4. If $k>1$ then $k+1$ and $k-1$ are both nonzero and there is no effect on the mean flow.

The critical radii for the 3 modes are related by

$$
r_{c_{k-1}}<r_{c}<r_{c_{k+1}}
$$

as shown in Figure 6.1. All three waves (with wavenumbers $k, k+1$ and $k-1$ ) experience critical layer absorption at their respective critical radii. This means that as the waves propagate outwards from the eye of the vortex, the $(k-1)$ mode is absorbed first at $r_{c_{k-1}}$ and its amplitude is greatly reduced while the other waves 
continue to propagate. Then the $k$ mode is absorbed at $r_{c}$ while the $(k+1)$ mode continues to propagate and is absorbed at $r_{c_{k+1}}$. This means that at $r_{c}$ there are only two components of the solution with non-negligible amplitude, corresponding to wavenumbers $k$ and $(k+1)$. In the critical layer around $r_{c}$, the inner layer solution for the mode $k$ is valid, but to obtain information about the effect that the $(k+1)$ mode has on the evolution of the critical layer around $r_{c}$, we need an outer solution for the $(k+1)$ mode.

In the critical layer near $r_{c}$, the $(k+1)$ mode is described by its outer solution. We write it as

$$
\psi^{(1 a)}(r, \lambda, t) \sim\left[\phi^{(1 a)}(r) e^{-i \omega t}+Y_{p a r t i c u l a r}\right] e^{i(k+1) \lambda}, \quad t \gg 1
$$

where $\phi^{1(a)}(r)$ is the solution of $(6.23)$, and can be written as a linear combination of the hypergeometric functions of the form

$$
\begin{aligned}
& f_{1(1)}(r) \sim r^{-(k+1)}\left[\left(1-\frac{r^{2}}{r_{c_{k+1}}^{2}}\right)+O\left(\left(1-\frac{r^{2}}{r_{c_{k+1}}^{2}}\right)^{2}\right)\right] \\
& f_{2(1)}(r) \sim r^{-k-1}\left[1-2\left(1-\frac{r^{2}}{r_{c_{k+1}}^{2}}\right) \log \left(1-\frac{r^{2}}{r_{c_{k+1}}^{2}}\right)+O\left(1-\frac{r^{2}}{r_{c_{k+1}}^{2}}\right)\right] .
\end{aligned}
$$

To find $Y_{\text {particular }}(r, t)$, we return to the nonhomogeneous equation (6.19) and substitute in the inner solution for $\phi^{(0)}(r, t)$ since we are considering the critical layer near $r_{c}$. The inner solution for $\phi^{(0)}(r, t)$ is (5.99). Substituting this into the right-hand side 


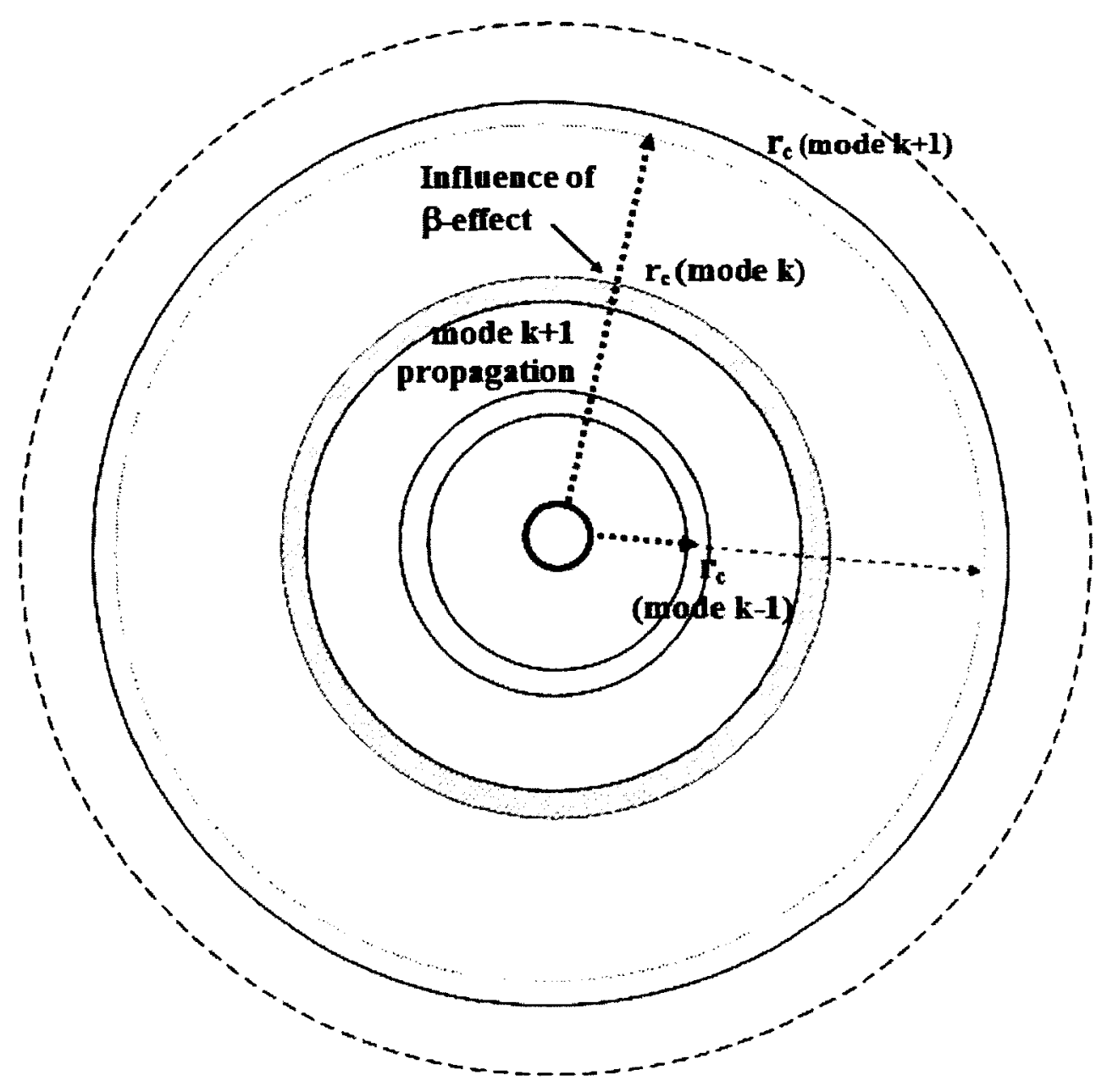

Figure 6.1: Schematic diagram of the $k, k-1, k+1$ modes propagation. Schematic diagram of the propagation of the waves with wavenumber $k$ (arising from the boundary forcing at $r=r_{1}$ ), and wavenumber $k \pm 1$ (arising from the $\beta$-effect). The shaded regions show the inner layers for the critical radii of each wave. The arrows show propagation of waves up to their critical radii. The wave with wavenumber $k+1$ is influenced by $\beta$ effect at the critical layer for wave with wavenumber $k$. 
of $(6.19)$ gives

$$
\begin{array}{r}
\frac{k}{r} \phi^{(0)}-\phi_{r}^{(0)} \sim e^{-i \omega t}\left(\frac{r}{r_{1}}\right)^{-k}\left(\frac{k}{r} h_{1}\left(r_{c}\right)-C(t) h_{1}\left(r_{c}\right) \frac{k}{r}\left(r-r_{c}\right)+C(t) h_{1}\left(r_{c}\right)+O(1 / t)\right. \\
\left.+\sum_{n=1}^{\infty} c_{n}\left[\left(r-r_{c}\right)(t)\right]^{n}\right), t \gg 1,(6
\end{array}
$$

where

$$
C(t)=\frac{4}{r_{c}}\left(\log \left(-i k \Omega_{0} \alpha r_{c}^{2} t\right)+\left(\gamma_{E}-1\right)\right)
$$

Then we use the variation of parameters formula to obtain the particular solution

$$
\begin{array}{r}
Y_{\text {particular }}(r, t) \sim\left(\int \frac{1}{W(r)} f_{1(1)}\left(\frac{k}{r} \phi^{(0)}-\phi_{r}^{(0)}\right) d r\right) f_{2(1)} \\
-\left(\int \frac{1}{W(r)} f_{2(1)}\left(\frac{k}{r} \phi^{(0)}-\phi_{r}^{(0)}\right) d r\right) f_{1(1)}, t \gg 1
\end{array}
$$

where $W(r)$ is the Wronskian of the two functions $f_{1(1)}$ and $f_{2(1)}$. So, the general solution for the nonhomogeneous equation can be written in the form

$$
\begin{gathered}
\phi^{(1 a)}(r, t) \sim\left(\int \frac{1}{W(r)} f_{1(1)}\left(\frac{k}{r} \phi^{(0)}-\phi_{r}^{(0)}\right) d r+A_{2}\right) f_{2(1)} \\
-\left(\int \frac{1}{W(r)} f_{2(1)}\left(\frac{k}{r} \phi^{(0)}-\phi_{r}^{(0)}\right) d r-A_{1}\right) f_{1(1)}, t \gg 1
\end{gathered}
$$

Since we are evaluating the solution near $r_{c}$, we write $(6.29)$ and $(6.30)$ in terms of $\left(r-r_{c}\right)$ and get

$$
\begin{array}{r}
f_{1(1)} \sim r^{-(k+1)}\left[1-2 \log \left(1-\frac{r^{2}}{r_{c_{k+1}}^{2}}\right)\left(1-\frac{r^{2}}{r_{c_{k+1}}^{2}}\right)+O\left(1-\frac{r^{2}}{r_{c_{k+1}}^{2}}\right)\right] \\
\sim r^{-(k+1)}\left[a_{0}+a_{1}\left(r-r_{c}\right)\right],\left|r-r_{c}\right|<1
\end{array}
$$


and

$$
f_{2(1)} \sim r^{-(k+1)}\left(1-\frac{r^{2}}{r_{c_{k+1}}^{2}}\right) \sim r^{-(k+1)}\left[a_{2}+a_{3}\left(r-r_{c}\right)\right],\left|r-r_{c}\right|<1,
$$

where

$$
\begin{array}{r}
a_{0}=1-2 \log \left(1-\frac{r_{c}^{2}}{r_{c_{k+1}}^{2}}\right)\left(1-\frac{r_{c}^{2}}{r_{c_{k+1}}^{2}}\right), \\
a_{1}=-\frac{2}{r_{c_{k+1}}^{2}}\left(2 \log \left(1-\frac{r_{c}^{2}}{r_{c_{k+1}}^{2}}\right)-1\right), \\
a_{2}=1-\frac{r_{c}^{2}}{r_{c_{k+1}}^{2}}, \\
a_{3}=-\frac{2 r_{c}}{r_{c_{k+1}}^{2}} .
\end{array}
$$

The Wronskian for the functions $f_{1(1)}$ and $f_{2(2)}$ is

$$
W(r)=\left|\begin{array}{ll}
f_{1(1)} & f_{2(1)} \\
f_{1(1)}^{\prime} & f_{2(1)}^{\prime}
\end{array}\right| \sim r^{-2(k+1)}\left(a_{0} a_{3}-a_{1} a_{2}\right),\left|r-r_{c}\right|<1 .
$$

So $\phi^{(1 a)}(r, t)$ is

$$
\begin{gathered}
\phi^{(1 a)}(r, t) \sim e^{-i \omega t}\left[\int \frac { ( a _ { 0 } + a _ { 1 } ( r - r _ { c } ) ) } { a _ { 0 } a _ { 3 } - a _ { 1 } a _ { 2 } } \frac { r ^ { ( 2 k + 2 ) } } { r ^ { ( 2 k + 1 ) } } \left(\frac{k}{r} h_{1}(r)-C(t) h_{1} \frac{k}{r}\left(r-r_{c}\right)\right.\right. \\
\left.\left.+C(t) h_{1}+\sum_{n=1}^{\infty} c_{n}\left(\left(r-r_{c}\right) t\right)^{n}\right) d r+A_{1}\right] r^{-k}\left[a_{2}+a_{3}\left(r-r_{c}\right)\right] \\
-\left[\int \frac { ( a _ { 2 } + a _ { 3 } ( r - r _ { c } ) ) } { a _ { 0 } a _ { 3 } - a _ { 1 } a _ { 2 } } \frac { r ^ { ( 2 k + 2 ) } } { r ^ { ( 2 k + 1 ) } } \left(\frac{k}{r} h_{1}(r)-C(t) h_{1} \frac{k}{r}\left(r-r_{c}\right)+C(t) h_{1}\right.\right. \\
\left.\left.+\sum_{n=1}^{\infty} c_{n}\left(\left(r-r_{c}\right) t\right)^{n}\right) d r+A_{2}\right] r^{-k}\left[a_{0}+a_{1}\left(r-r_{c}\right)\right],\left|r-r_{c}\right|<1, t \gg 1 .
\end{gathered}
$$


To find $\phi^{(1 a)}(r)$ we have to evaluate the integrals in (6.39). Note that

$$
\int \sum_{n=1}^{\infty} c_{n}\left[\left(r-r_{c}\right) t\right]^{n} d r=\int \sum_{n=1}^{\infty} c_{n}\left[\left(r-r_{c}\right) t\right]^{n} d r=\frac{1}{t} \sum_{n=2}^{\infty} c_{n}^{\prime}\left[\left(r-r_{c}\right) t\right]^{n} \sim O\left(\frac{1}{t}\right)
$$

After integrating (6.39) we obtain

$$
\phi^{(1 a)}(r, t) \sim e^{-i \omega t} r^{(-k)}\left(A_{1}\left[a_{2}+a_{3}\left(r-r_{c}\right)\right]-A_{2}\left[a_{0}+a_{1}\left(r-r_{c}\right)\right]+\frac{2}{3} r^{2} C(t)\right)
$$

or,

$$
\phi^{(1 a)}(r, t) \sim\left[O(1)+O\left(r-r_{c}\right)+O(\log t)\right] e^{-i \omega t}
$$

This solution represents the leading-order contribution to the solution near the critical layer (near $r_{c}$ ), arising from the variation of the Coriolis parameter. The wave amplitude increases as $\log t$ because of the $\beta$-effect, and it is the result of interaction between the $k$ mode and the $k+1$ mode in the critical layer for the $k$ mode.

\subsection{Combined effects of nonlinearity and the $\beta$ - effect}

We now consider equation (3.52) which includes both nonlinearity and the $\beta$-effect.

$$
\begin{array}{r}
\left(\frac{\partial}{\partial t}+\frac{\vec{v}}{r} \frac{\partial}{\partial \lambda}\right) \nabla^{2} \psi-\frac{1}{r} \psi_{\lambda} \frac{\partial}{\partial r}\left(\vec{v}_{r}+\frac{1}{r} \bar{v}\right)-\frac{\beta}{r} \psi_{\lambda} \sin \lambda+\frac{\beta}{\epsilon} \bar{v} \cos \lambda+\beta \psi_{r} \cos \lambda \\
=-\frac{\varepsilon}{r}\left(\psi_{r} \nabla^{2} \psi_{\lambda}-\psi_{\lambda} \nabla^{2} \psi_{r}\right)
\end{array}
$$

The parameters $\varepsilon$ and $\beta$ are both small, the solution of (6.43) can be written as

$$
\psi \sim \psi^{(0)}+\frac{\beta}{\varepsilon} \psi^{(\tilde{B})}+\varepsilon \psi^{(1)}+\beta \psi^{(1 a)}+\beta \psi^{(1 b)}+O\left(\varepsilon^{2}\right)+O(\varepsilon \beta)+O\left(\beta^{2}\right)
$$


where $\psi^{(0)}, \psi^{(\tilde{B})}, \psi^{(1)}, \psi^{(1 a)}, \psi^{(1 b)}$ are functions of $r, \lambda$, and $t$.

As noted in chapter 3 and section 6.2 , with the reference length scale for our problem it is reasonable to consider the situation where $\beta \ll \varepsilon \ll 1$. In that case $\beta \varepsilon^{-1} \ll 1$ and so the $\beta \varepsilon^{-1}$ term that arises in the solution is not a leading order term. We first investigate the $O(\varepsilon)$ and $O(\beta)$ terms in the solution and then at the end of this section we will add the $\beta \varepsilon^{-1}$ term to the analysis.

We can rewrite equation $(6.43)$ in the form

$$
L(\psi)+\beta B(\psi)=-\varepsilon N(\psi, \psi)
$$

where $L, B$, and $N$ are the partial differential operators given by

$$
\begin{gathered}
L(\psi)=\left(\frac{\partial}{\partial t}+\frac{\bar{v}}{r} \frac{\partial}{\partial \lambda}\right) \nabla^{2} \psi-\frac{1}{r} \psi_{\lambda} \frac{\partial}{\partial r}\left(\bar{v}_{r}+\frac{1}{r} \bar{v}\right) \\
N\left(\psi_{1}, \psi_{2}\right)=-\frac{\varepsilon}{r}\left(\psi_{1 r} \nabla^{2} \psi_{2 \lambda}-\psi_{1 \lambda} \nabla^{2} \psi_{2 r}\right) \\
B(\psi)=-\frac{1}{r} \psi_{\lambda} \sin \lambda+\psi_{r} \cos \lambda=-\frac{1}{2 i} \psi_{\lambda}\left(e^{i \lambda}-e^{-i \lambda}\right)+\frac{1}{2} \psi_{r}\left(e^{i \lambda}+e^{-i \lambda}\right) .
\end{gathered}
$$

Substituting (6.44) into equation (6.45), we obtain

$$
\begin{gathered}
L\left(\psi^{(0)}\right)+\beta L\left(\psi^{(1 a)}\right)+\beta L\left(\psi^{(1 b)}\right)+\varepsilon L\left(\psi^{(1)}\right)+\beta B\left(\psi^{(0)}\right)+\varepsilon \beta B\left(\psi^{(1)}\right) \\
=-\varepsilon N\left(\psi^{(0)}, \psi^{(0)}\right)-\varepsilon \beta N\left(\psi^{(0)}, \psi^{(1 a)}\right)-\varepsilon \beta N\left(\psi^{(0)}, \psi^{(1 b)}\right) \\
-\varepsilon^{2} N\left(\psi^{(0)}, \psi^{(1)}\right)+O\left(\varepsilon^{2} \beta\right)+O\left(\varepsilon \beta^{2}\right)+O\left(\varepsilon^{3}\right)+O\left(\beta^{3}\right) .
\end{gathered}
$$

To analyze the order of the different terms in (6.49), their wavenumbers and their possible interactions, we first note that at leading order $L\left(\psi^{(0)}\right)=0$, where $\psi^{(0)}$ is the linear solution defined in chapter 5 without the $\beta$-effect and corresponds to the modes $\pm k$ specified at the forced boundary $r=r_{1}$. We note also that the $O(\varepsilon)$ term 
corresponds to wavenumbers 0 and $\pm 2 k$, as shown in section 6.1 . The $O(\beta)$ term corresponds to wavenumbers $\pm(k+1)$ and $\pm(k-1)$, as shown in section 6.2 , the $O\left(\varepsilon^{2}\right)$ term corresponds to wavenumbers $\pm k$ and $\pm 3 k$, the $O(\varepsilon \beta)$ term corresponds to wavenumbers $\pm 1, \pm(2 k+1)$ and $\pm(2 k-1)$, and the $O\left(\beta^{2}\right)$ term corresponds to wavenumbers $\pm k, \pm(k+2)$ and $\pm(k-2)$. This information is summarized in Table 6.1 .

If $\beta \sim \varepsilon, \varepsilon \ll 1$ and $\beta \ll 1$, then $\varepsilon$ and $\beta$ are the same order of magnitude and the solution up to $O(\varepsilon)$ and $O(\beta)$ is simply the sum of the solutions obtained respectively in sections 6.1 and 6.2 .

Table 6.1: Wavenumbers corresponding to the terms in equation (6.49)

\begin{tabular}{cccc}
\hline \hline order & terms & wavenumber & $\begin{array}{c}\text { wavenumber } \\
\text { special case } k=1\end{array}$ \\
\hline$\varepsilon$ & $N\left(\psi^{(0)}, \psi^{(0)}\right) ; \quad L\left(\psi^{(1)}\right)$ & $0 ; \pm 2 k$ & $0, \pm 2$ \\
$\beta$ & $L\left(\psi^{(1 a)}\right) ; \quad L\left(\psi^{(1 b)}\right) ; \quad B\left(\psi^{(0)}\right)$ & $\pm(k-1) ; \pm(k+1)$ & $0, \pm 2$ \\
$\beta^{2}$ & $B\left(\psi^{(1 a)}\right) ; \quad B\left(\psi^{(1 b)}\right)$ & $\pm(k-2) ; \pm k ; \pm(k+2)$ & $\pm 1, \pm 3$ \\
$\varepsilon \beta$ & $B\left(\psi^{(1)}\right)$ & $\pm 1 ; \pm(2 k-1) ; \pm(2 k+1)$ & $\pm 1, \pm 3$ \\
$\varepsilon^{2}$ & $N\left(\psi^{(0)}, \psi^{(1 a)}\right) ; N\left(\psi^{(0)}, \psi^{(1 b)}\right)$ & $\pm 1 ; \pm(2 k-1) ; \pm(2 k+1)$ & $\pm 1, \pm 3$ \\
\hline & $N\left(\psi^{(0)}, \psi^{(1)}\right)$ & $\pm k ; \pm 3 k$ & $\pm 1, \pm 3$ \\
\hline
\end{tabular}

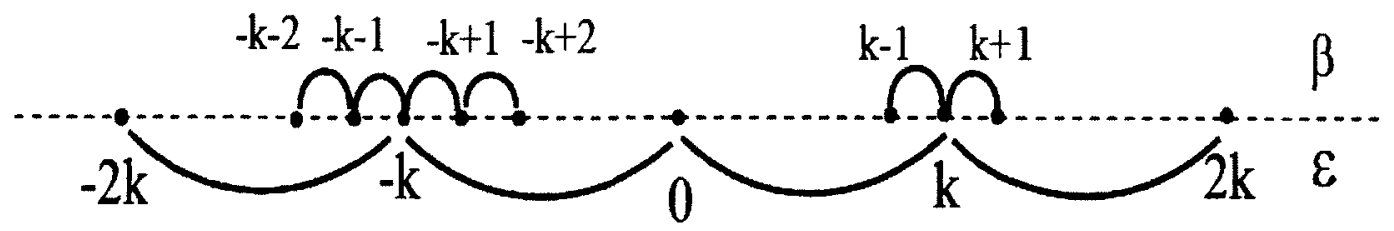

Figure 6.2: Schematic diagram for wavenumbers. Schematic diagram for the wavenumbers in equation (6.49) of the terms of $O(\varepsilon)$ and $O(\beta)$, the case where $k>0$, $k \neq 1$. The $O(\varepsilon)$ terms include a component with zero wave number, but $O(\beta)$ do not. There is no interaction between the $\varepsilon$ and $\beta$ terms in the mean flow. 
If $k=1$, then the $O(\varepsilon)$ and $O(\beta)$ terms both correspond to the same wavenumbers, 0 and \pm 2 .

If $\beta \ll \varepsilon \ll 1$ then the solution can be written in the form

$$
\psi \sim \psi^{(0)}+\varepsilon \psi^{(1)}+\beta \psi^{(1 a)}+\beta \psi^{(1 b)}+O(\varepsilon \beta)+O\left(\varepsilon^{2}\right)+O\left(\beta^{2}\right)
$$

Substituting this equation into (6.49) we obtain

$$
\begin{array}{ll}
O(1): & L\left(\psi^{(0)}\right)=0 \\
O(\varepsilon): & L\left(\psi^{(1)}\right)=-N\left(\psi^{(0)}, \psi^{(0)}\right), \\
O(\beta): & L\left(\psi^{(1 a)}\right)+L\left(\psi^{(1 b)}\right)=-B\left(\psi^{(0)}\right), \\
O(\varepsilon \beta): & L\left(\psi^{(2 \varepsilon \beta)}\right)=-N\left(\psi^{(0)}, \psi^{(1 a)}\right)-N\left(\psi^{(0)}, \psi^{(1 b)}\right)-B\left(\psi^{(1)}\right), \\
O\left(\varepsilon^{2}\right): & L\left(\psi^{(2 \varepsilon)}\right)=-N\left(\psi^{(0)}, \psi^{(1)}\right)-N\left(\psi^{(1)}, \psi^{(0)}\right), \\
O\left(\beta^{2}\right): & L\left(\psi^{(2 \beta)}\right)=-B\left(\psi^{(1 a)}+\psi^{(1 b)}\right) .
\end{array}
$$

If $k>1$, then, up to the orders shown in the table, there are no interactions between the terms of each order because they all correspond to different wavenumbers. The larger the value of $k$ is, the further apart the wavenumbers for the $O(\varepsilon)$ and $O(\beta)$ terms are. This situation is illustrated in Figure 6.2. Continuing to higher orders of $\varepsilon$ and $\beta$ the solution can be represented as a discrete spectrum of contributions from several wavenumbers. Since the $O(\varepsilon)$ term includes a zero wavenumber component, it means that nonlinearity affects the mean flow. With $k>1$, however, the $O(\beta)$ term has no zero wavenumber component, so the $\beta$-effect does not affect the mean flow. The $O\left(\beta^{2}\right)$ and $O\left(\varepsilon^{2}\right)$ include terms corresponding to the fundamental wavenumber $\pm k$. All the $O\left(\beta^{2}\right), O(\varepsilon \beta)$ and $O\left(\varepsilon^{2}\right)$ terms contribute higher wavenumbers to the solution. Figure 6.2 demonstrates that there are no further contributions to the zero 
wavenumber component until higher orders. So we can conclude that if $k>1$ the mean flow is influenced only by nonlinearity.

The only situation where terms of different orders have the same wavenumbers is the special case where $k=1$. In that case the $O(\varepsilon)$ and $O(\beta)$ terms both correspond to the wavenumbers $k=0$ and $k=2$, and so the solution up to this order is the sum of both effects. Since the $O(\varepsilon)$ and $O(\beta)$ terms both have zero wavenumber components, we conclude that both nonlinearity and the $\beta$-effect influence the mean flow. The evaluation of the mean flow is discussed in section 6.4 .

We now investigate the $O\left(\beta \varepsilon^{-1}\right)$ term in the solution

$$
\psi \sim \psi^{(0)}+\frac{\beta}{\varepsilon} \psi^{(\tilde{B})}+\varepsilon \psi^{(1)}+\beta \psi^{(1 a)}+\beta \psi^{(1 b)}+O\left(\varepsilon^{2}\right)+O(\varepsilon \beta)+O\left(\beta^{2}\right)
$$

The function $\psi^{(\bar{B})}(r, \lambda, t)$ satisfies the equation

$$
O(\beta / \varepsilon): \quad L\left(\psi^{(\tilde{B})}\right)=-\frac{\bar{v}}{2}\left(e^{i \lambda}+e^{-i \lambda}\right)
$$

Thus $\psi^{(\tilde{B})}(r, \lambda, t)$ corresponds to a wavenumber of 1 and must take the form

$$
\psi^{(\tilde{B})}=\phi^{\tilde{B}}(r, t) e^{i \lambda}+\text { c.c. }
$$

with the boundary condition $\phi^{\tilde{B}}\left(r_{1}, t\right)=0$. The solution of the corresponding homogeneous equation will take the same general form as that obtained for the leading order solution and can be expressed in terms of hypergeometric functions as it was done in chapter 4 . The solution has the same qualitative form as the steady solution $\Phi_{\infty}$. Since the nonhomogeneous term is independent of time, we can find a steady particular solution to this equation. Because of the zero boundary condition, the homogeneous solution should be steady as well. So the function $\phi^{\tilde{B}}$ is steady and 
satisfies the equation

$$
\phi_{r r}^{\bar{B}}+\frac{\phi_{r}^{\bar{B}}}{r}-\frac{1}{r^{2}} \phi^{\bar{B}}+\frac{\frac{1}{r} \frac{\partial}{\partial r}\left(\bar{v}_{r}+\bar{v} / r\right)}{\omega-\bar{\Omega} k} \phi^{\bar{B}}=-\frac{\bar{v}}{2}
$$

Its solution has only a quantitative effect on the solution for the modes \pm 1 . If $k=1$, $\phi^{\tilde{B}}$ has the same wavenumber as the fundamental mode. If $k \neq 1$, then the $O\left(\beta \varepsilon^{-1}\right)$ term does not interact with the fundamental mode or any of the $O(\varepsilon)$ terms (which correspond to wavenumbers $0, \pm k, \pm 2 k$ ).

\subsection{Evolution of the mean flow in the inner layer}

From equation (6.7) we see that the mean flow changes with time due to the nonlinear interaction. We also saw in section 6.3 that in the case where $k=1$, the $\beta$-effect contributes to the mean flow change. In that case the change in the mean angular velocity is the sum of the changes due to the nonlinear and $\beta$ effects. Let us consider first the change of the mean flow that results from the nonlinear wave-mean flow interaction.

According to (3.49) the total streamfunction is

$$
\Psi(r, \lambda, t)=\bar{\psi}(r)+\varepsilon \psi(r, \lambda, t)
$$

The perturbation $\varepsilon \psi(r, \lambda, t)$ now includes a zero wave number component which we will call $\psi_{0}(r, t)$. Thus the mean streamfunction at time $t$ is the sum of the initial mean streamfunction $\bar{\psi}(r)$ and the time-dependent mean streamfunction $\psi_{0}(r, t)$ induced by the nonlinear interaction. We let $\bar{\psi}_{\text {total }}(r, t)$ be the mean streamfunction at time $t$. Then we can write

$$
\bar{\psi}_{\text {total }}(r, t)=\bar{\psi}(r)+\psi_{0}(r, t)
$$


Similarly, the mean azimuthal velocity is

$$
\bar{v}_{t o t a l}(r, t)=\bar{v}(r)+v_{0}(r, t)
$$

where $v_{0}(r, t)$ is the wave-induced mean velocity. We can obtain an equation for the evolution of the mean flow either from equation (6.7) or directly from the azimuthal momentum equation as done in Appendix B. In either case, we end up with (B.16):

$$
\frac{\partial \tilde{v}_{\text {total }}}{\partial t}=\varepsilon^{2} \frac{1}{r^{2}} \frac{\partial}{\partial r}\left(2 k r \operatorname{Im}\left(\phi^{(0)} \phi_{r}^{*(0)}\right)\right)
$$

where $\bar{v}_{\text {total }}$ is the azimuthal velocity of the main flow.

To find the change in the azimuthal velocity in the inner layer where $\left|r-r_{c}\right| t<1$ we use the leading-order solution $\phi^{(0)}(r, t)(5.99)$

$$
\begin{array}{r}
\phi^{(0)} \sim e^{-i \omega t}\left(\frac{r_{c}}{r_{1}}\right)^{-k} h_{1}\left(1+b_{1}\left(r_{c}-r\right)-i b_{2} \frac{\pi}{2}\left(r_{c}-r\right)\right. \\
\left.+b_{2}\left(r_{c}-r\right) \log (t)-i \frac{b_{3}}{t}+i b_{4} t\left(r-r_{c}\right)^{2}+O\left(\left(r-r_{c}\right)^{3} t^{2}\right)\right)
\end{array}
$$

where $b_{i}$ for $i=1,2,3,4$ are real numbers which are defined as

$$
\begin{array}{r}
b_{1}=\frac{4}{r_{c}}\left(\log \left(k \Omega_{0} \alpha r_{c}^{2}\right)+\gamma_{E}-1\right), \\
b_{2}=\frac{4}{r_{c}}, \\
b_{3}=\frac{2}{k \Omega_{0} \alpha r_{c}^{2}}, \\
b_{4}=8 k \Omega_{0} \alpha .
\end{array}
$$


The derivative of $\phi^{*(0)}$ with respect to $r$ in the inner layer is

$$
\begin{array}{r}
\phi_{r}^{*(0)} \sim e^{i \omega t}\left(\frac{r_{c}}{r_{1}}\right)^{-k} h_{1}\left(-b_{1}-i b_{2} \frac{\pi}{2}-b_{2} \log (t)\right. \\
\left.-2 i b_{4}\left(r_{c}-r\right) t+O\left(\left(r-r_{c}\right)^{2} t^{2}\right)\right)
\end{array}
$$

Substituting this into (6.59) gives

$$
\frac{\partial \bar{v}_{\text {total }}}{\partial t} \sim \varepsilon^{2} \frac{2 k}{r_{c}^{2}}\left(\frac{r_{c}}{r_{1}}\right)^{-2 k}\left(-b_{2} \frac{\pi}{2}+b_{2} b_{3} \frac{\log (t)}{t}+O\left(\left(r-r_{c}\right)^{2} t^{2}\right)\right)
$$

So the time derivative of the mean azimuthal velocity satisfies

$$
\left|\frac{\partial \bar{v}_{\text {total }}}{\partial t}\right| \sim \varepsilon^{2}\left(O(1)+O\left(\frac{\log t}{t}\right)+O\left(t^{2}\left(r-r_{c}\right)^{2}\right)\right)
$$

Integrating with respect to time from the initial time to time $t$ gives an equation for the azimuthal velocity of the mean flow in the critical layer as a function of time $t$,

$$
\bar{v}_{t o t a l}(r, t) \sim \bar{v}(r)-\varepsilon^{2} \frac{2 k}{r_{c}^{3}} h_{1}^{2}\left(\frac{4}{r_{c}} \frac{\pi}{2} t-\frac{8}{k \Omega_{0} \alpha r_{c}^{3}}(\log t)^{2}\right)
$$

So we see that the azimuthal velocity decreases with time.

We saw in section 6.3 that in the case where $k=1$, nonlinearity and the $\beta$-effect both influence the mean flow. In this case we find that

$$
\bar{v}_{\text {total }}(r, t) \sim \bar{v}(r)-\varepsilon^{2} \frac{2 k}{r_{c}^{3}} h_{1}^{2}\left(\frac{4}{r_{c}} \frac{\pi}{2} t+O(\log t)^{2}\right)+\varepsilon \beta O(\log t) e^{i \omega t}+\text { c.c. }
$$

So the mean flow change consists of two parts: a term decreasing with time and some oscillations with amplitude proportional to $\log t$. This change in the azimuthal velocity means that the mean angular velocity $\bar{\Omega}_{\text {total }}(r, t)$ also decreases with time in the critical layer and hence the location of the critical radius changes with time. 
For any initial $\bar{\Omega}_{\text {total }}(r)$ profile that decreases monotonically with $r$ (such as the profile $\bar{\Omega}_{\text {total }}(r, 0)=\bar{\Omega}(r)=\Omega_{0}\left(1-\alpha r^{2}\right)$ used in our study), a decrease in $\bar{\Omega}_{\text {total }}(r, t)$ means that the critical radius moves inwards, towards the centre of the vortex. This is seen in Figure 6.3. This result is in agreement with hurricane observations where the secondary eyewall moves toward the centre of the hurricane (see, e.g., Sitkowski et al., 2011).

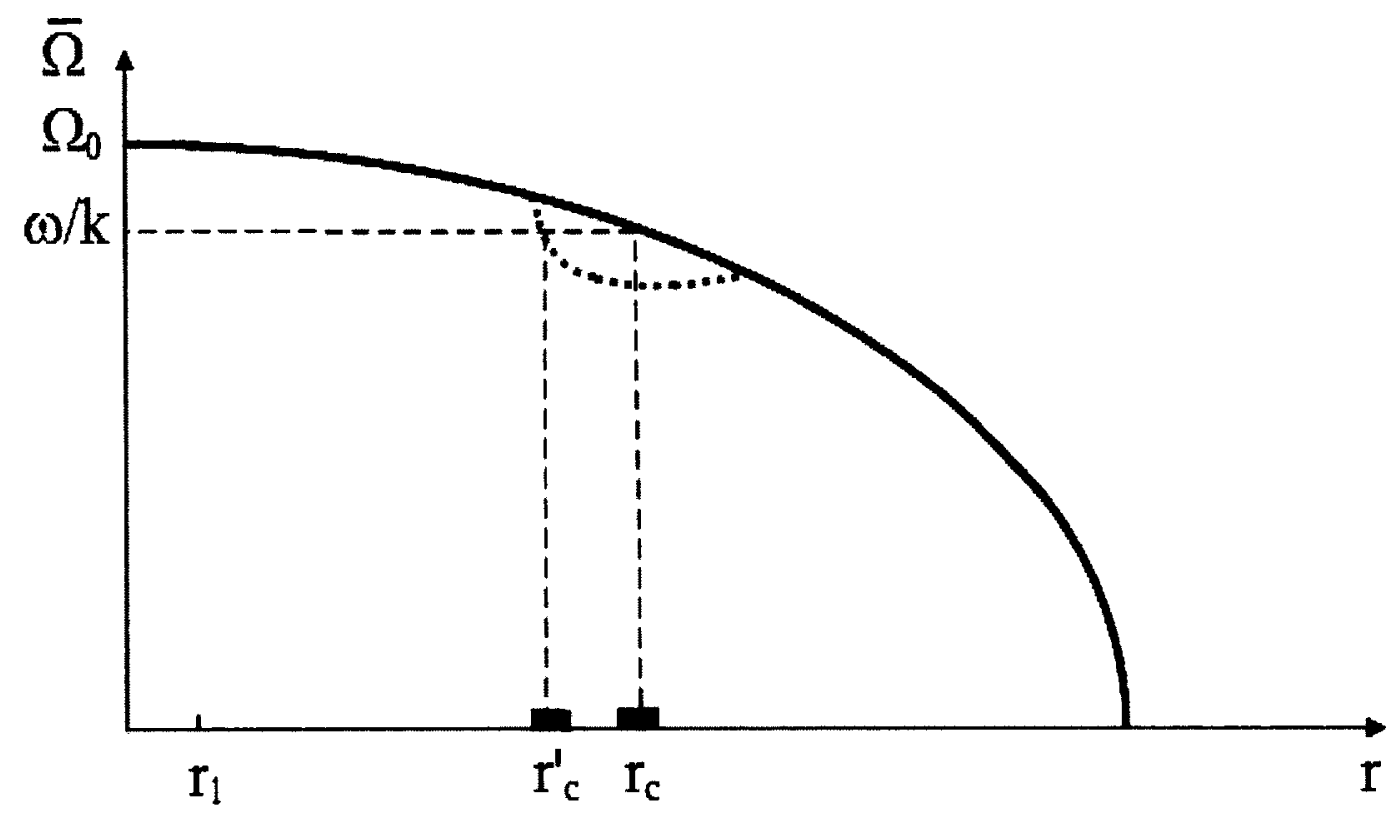

Figure 6.3: Dynamics of the critical radius location. The initial critical radius is given by $\bar{\Omega}=\omega / k$, where $\bar{\Omega}(r)$ is the initial angular velocity. If the angular velocity decreases in the critical layer near $r_{c}$, as shown by the dashed line, the critical radius moves towards the centre of the vortex, from location $r_{c}$ to $r_{c}^{\prime}<r_{c}$. 


\section{Chapter 7}

\section{Conclusions}

\subsection{Summary}

In this thesis we presented approximate asymptotic solutions for a problem representing vortex Rossby waves propagation in a tropical cyclone. The cyclone was considered on a horizontal plane defined by polar coordinates $r$ and $\lambda$. The cyclone was represented as a vortex with angular velocity $\bar{\Omega}(r)$ in a domain given by $r_{1} \leq r<\infty$ and $0 \leq \lambda<2 \pi$. At $r=r_{1}$, a sinusoidal wave was generated by a boundary condition $\psi\left(r_{1}, \lambda, t\right)=e^{i(k \lambda-\omega t)}$. We considered waves propagating outwards in the cyclone in the form $\psi(r, \lambda, t)=\phi(r, t) e^{i k \lambda}+\phi^{*}(r, t) e^{-i k \lambda}$. We derived equations for the wave amplitude starting from the governing equations for fluid dynamics in a coordinate frame rotating with the Earth. Several different configurations were examined.

First, we considered the linear problem where the wave amplitude $\phi$ is independent on $t$. In this case $\phi$ is given by an ordinary differential equation in $r$, which is singular at $r_{c}$, where the phase speed of the wave mode is equal to the angular velocity of the cyclone rotation. This equation was solved for a quadratic profile of the angular velocity. The solution demonstrates the attenuation of the waves in the cyclone at 
the critical radius.

Next we studied the linear time-dependent problem where the wave amplitude $\phi$ depends on both $r$ and $t$. We derived the solutions valid for late time for the different regions in the tropical cyclone: the region between the eyewall and the critical layer, the region inside the critical layer, and the region outside the critical layer, far from the centre of the vortex. These solutions were expressed in terms of hypergeometric functions. Each solution comprises a term with steady amplitude and some timedependent terms that go to zero as $t \rightarrow \infty$.

To find a nonsingular solution near the critical radius $r=r_{c}$, we introduced an inner layer and an inner variable $Z=\left(r-r_{c}\right) / \mu$ with a corresponding slow time variable $T=\mu t$, where $\mu$ is the thickness of the inner layer. We found that the inner solution contains terms proportional to $\log (t)$. Then we reintroduced the nonlinear terms to the problem and observed that these terms would grow like $t^{2}$ in both the outer and inner solutions. This means that the nonlinear solution is valid for $t<$ $O\left(\varepsilon^{-1 / 2}\right)$. The nonlinear effects arise in the wave mode with wavenumber $2 k$ and in the mean flow with wavenumber zero.

We also added a $\beta$-term into consideration to evaluate the effects of the variation of the Coriolis force on the vortex Rossby wave dynamics. Our analyses showed that the $\beta$ term gives rise to wave modes with wavenumbers $(k+1)$ and $(k-1)$. All three waves, modes $(k+1), k$ and $(k-1)$, experience critical layer absorption at their respective critical radii. While the waves propagate outwards from the eye of the cyclone, the $(k-1)$ mode is absorbed first at its critical layer $r_{c_{k-1}}$ and its amplitude is reduced at this radius. The $k$ mode is absorbed at $r_{c}$ while the $(k+1)$ mode continues to propagate and is absorbed at $r_{c_{k}+1}$. So there are two modes, with wavenumbers $k$ and $(k+1)$, in the critical layer $r_{c}$ and the wave amplitude of the $k+1$ mode grows like $\log (t)$ in the vicinity of the critical layer $r_{c}$. We also considered the more general 
configuration that includes both nonlinearity and the $\beta$-effect.

We concluded that if the fundamental mode has wavenumber $k>1$, the $\beta$-effect does not influence the mean flow. If $k=1$ then the mean flow is influenced by both effects. The azimuthal velocity of the mean flow decreases with time because of the nonlinear interaction, and the $\beta$-effect adds oscillations to the mean flow.

The main features of our solutions, namely the critical layer absorption of the waves, the development of concentric rings of high wave activity and the changes in the location of these rings with time, can be used to explain some of the observed characteristics of tropical cyclones, such as the formation and evolution of the secondary eyewall.

An important issue in the study of vortex Rossby wave dynamics in tropical cyclones is the interaction between the waves and the mean flow in the vortex. The problem of wave-mean flow interaction has been studied by many previous researchers in the context of barotropic Rossby waves in a rectangular domain (Dickinson, 1970, Warn and Warn, 1976, Warn and Warn, 1978, Campbell and Maslowe, 1998, Campbell, 2004). We see some similarities in our conclusions compared with these previous studies. They found that wave-mean flow interactions occur in the critical layer, and the thickness of the critical layer is $O\left(\varepsilon^{-1 / 2}\right)$ which is similar to our conclusions for the vortex problem. In both problems there is also the phase shift in the logarithmic term of the solution which defines the attenuation in the wave amplitude. The timedependent solution also has similar features. The mean flow changes with time due to nonlinearity.

It is interesting to note that in rectangular coordinates these features of the solution arise because of the $\beta$-effect. In the context of vortex dynamics these effects are obtained even without the inclusion of the $\beta$-effect.

Our results give some insight into the secondary eyewall cycle. We have identified 
at least two mechanisms that could result in changes in the initial eyewall configuration. One is the development of multiple concentric rings of critical layers at different orders, due to the inclusion of the $\beta$-effect; these could be seen as concentric secondary eyewalls. Another observation is the modification of the mean flow by the wave momentum flux divergence which changes the location of the critical radius for the $O(1)$ wave (the strongest of the concentric eyewalls) for time scales up to $t \sim O\left(\varepsilon^{-1 / 2}\right)$. For longer time scales when the nonlinear terms have become the same order as the linear terms, a late-time solution may be possible through the introduction of a "slow" time variable. This is one of the possibilities for further work on this problem. Numerical simulations can also be used to shed further light on the evolution of the waves and their effects on the mean flow.

A possible further extension of this problem would be the introduction of a vertical coordinate, making the problem three-dimensional and allowing the introduction of internal gravity waves in addition to vortex Rossby waves. There has been considerable debate in the past few decades as to which type of waves, vortex Rossby waves or vortex gravity waves, play a greater role in hurricane dynamics. Brunet and Montgomery (2002), and Schecter and Montgomery (2004) discussed the influence of inertia-buoyancy oscillations, i.e. gravity waves, on the vortex dynamics. They conclude that these oscillations are important for cyclones in the middle latitudes. Schecter and Montgomery (2004) showed that the influence of vortex waves exceeds the effect of gravity wave propagation on the vortex dynamics. Hendricks et al. (2010) included the inertia-gravity waves in their numerical simulations of the vortical motion in a hurricane. They concluded that inertia-gravity waves are insignificant in the process of intensification or decay of the vortex. An investigation that includes both types of waves could provide some insight to help address this question. 


\section{Appendix A}

\section{Hypergeometric functions}

\section{A.1 Generalized hypergeometric functions}

The generalized hypergeometric function has the form (see Erdelyi, 1953)

$$
{ }_{p} F_{q}\left(a_{1}, a_{2} \ldots, a_{p} ; b_{1}, b_{2}, \ldots, b_{q} ; z\right)=\sum_{n=0}^{\infty} \frac{\left(a_{1}\right)_{n} \ldots\left(a_{p}\right)_{n}}{\left(b_{1}\right)_{n} \ldots\left(b_{q}\right)_{n} n !} z^{n}
$$

where $z$ is a complex variable, $a_{k}$, with $k=1, \ldots, p$, and $b_{j}$ with $j=1, \ldots, q$, are complex constants. The notation $(a)_{n}$ is the Pochhammer symbol,

$$
(a)_{n}=\Pi_{i=0}^{n-1}(a+i)
$$

and $(a)_{0}=1,(0)_{0}=1$. For the case where $p=2$ and $q=1$, the generalized hypergeometric function is called simply the hypergeometric function $F$ and is given by

$$
F={ }_{2} F_{1}\left(a_{1}, a_{2} ; b_{1} ; z\right)=\sum_{n=0}^{\infty} \frac{\left(a_{1}\right)_{n}\left(a_{2}\right)_{n}}{\left(b_{1}\right)_{n} n !} z^{n}
$$




\section{A.2 The solutions of the hypergeometric equation}

The hypergeometric function (A.3) is a solution of the hypergeometric differential equation

$$
z(1-z) f^{\prime \prime}+(c-(a+b+1) z) f^{\prime}-a b f=0
$$

where $z$ is a complex variable, and $a, b$ and $c$ are complex constants. This equation has three regular singular points, at $z=0,1, \infty$ (see Abramowitz and Stegun, 1964). The solutions depend on the values of the constants $a, b$, and $c$. In our problem (chapter $4, \mathrm{p} .55), c=a+b+1=p+1, a b=-2$. We find that $p=-k$ or $p=k$ so the values of $a$ and $b$ are

$$
\begin{array}{r}
a=\frac{k+\sqrt{k^{2}+8}}{2} \\
b=\frac{k-\sqrt{k^{2}+8}}{2} \\
c=k+1
\end{array}
$$

for $p=k$. And

$$
\begin{array}{r}
a=\frac{-k+\sqrt{k^{2}+8}}{2} \\
b=\frac{-k-\sqrt{k^{2}+8}}{2} \\
c=-k+1
\end{array}
$$

for $p=-k$. Near the singular point $z=0$ equation (A.4) has two linearly independent solutions valid for $|z|<1$ (equations 15.5.18 and 15.5.19 from Abramowitz and Stegun, 
1964). These solutions are

$$
\begin{gathered}
f_{1(0)}(z)=F(a, b ; 1+k, z)=F\left(\frac{k+\sqrt{k^{2}+8}}{2}, \frac{k-\sqrt{k^{2}+8}}{2}, 1+k, z\right) \\
\sum_{n=0}^{\infty} \frac{\left(\frac{k+\sqrt{k^{2}+8}}{2}\right)_{n}\left(\frac{k-\sqrt{k^{2}+8}}{2}\right)_{n}}{(1+k)_{n} n !} z^{n}, \\
f_{2(0)}=F(a, b ; 1+k, z) \log z+\sum_{n=1}^{\infty} \frac{(a)_{n}(b)_{n}}{(1+k)_{n} n !} z^{n} \\
{[\chi(a+n)-\chi(a)+\chi(b+n)-\chi(b)-} \\
\chi(k+1+n)+\chi(k+1)-\chi(n+1)+\chi(1)] \\
-\sum_{n=0}^{k} \frac{(n-1) !(-k)_{n}}{(1-a)_{n}(1-b)_{n}} z^{-n} .
\end{gathered}
$$

$F(a ; b ; c ; z)$ in solutions (A.7) and (A.8) is a hypergeometric function which is defined by the formula

$$
F(a ; b ; c ; z)=\sum_{n=0}^{\infty} \frac{(a)_{n}(b)_{n}}{n !(c)_{n}} z^{n}
$$

where $(a)_{n}$ is the Pochhammer symbol,

$$
(a)_{n}=\Pi_{i=0}^{n-1}(a+i)
$$

with $(a)_{0}=1,(0)_{0}=1$. In our problem the singular point $z=0$ is not in the domain of the problem. So we do not need solutions (A.7) and (A.8).

Near the other singular point $z=1$ we introduce a new variable $z_{1}=1-z$ and write (A.4) as

$$
z_{1}\left(1-z_{1}\right) f^{\prime \prime}+(0-(-k+1)) z_{1} f^{\prime}-2 f=0
$$

In this case the solution can be written following equations (15.5.20) and (15.5.21) in 
Abramowitz and Stegun (1964) for the case where $c=0$. For $p=-k$ two linearly independent solutions of the hypergeometric equation (4.34), valid for $|z-1|<1$, are

$$
f_{1(1)}(1-z)=f_{1(1)}\left(z_{1}\right)=(1-z) F(a+1, b+1 ; 2 ;(1-z))
$$

and

$$
\begin{gathered}
f_{2(1)}(1-z)=f_{2(1)}\left(z_{1}\right)=(1-z) F(a+1, b+1 ; 2 ;(1-z)) \log (1-z) \\
+(1-z) \sum_{n=1}^{\infty} \frac{(a+1)_{(n)}(b+1)_{(n)}}{(2)_{(n)} n !}(1-z)^{n} \\
{[\chi(a+1+n)-\chi(a+1)+\chi(b+1+n)-\chi(b+1)-} \\
\chi(2+n)+\chi(2)-\chi(n+1)+\chi(1)]+\frac{1}{a b},
\end{gathered}
$$

where

$$
\chi(z)=\frac{d \log (\Gamma(z))}{d z} .
$$

These solutions are valid for $0<|z|<2,|z| \neq 1$. In our problem $a$ and $b$ are defined by (A.5) and (A.6). For $p= \pm k$ these solutions can be written as

$$
f_{1(1)}(1-z)=(1-z) \sum_{n=0}^{\infty} \frac{\left(\frac{ \pm k+\sqrt{k^{2}+8}}{2}+1\right)_{(n)}\left(\frac{ \pm k-\sqrt{k^{2}+8}}{2}+1\right)_{(n)}}{(2)_{(n)} n !}(1-z)^{n}
$$


and

$$
\begin{aligned}
f_{2(1)}(1-z) & =-\frac{1}{2} \\
+ & (1-z) \sum_{n=0}^{\infty} \frac{\left(\frac{ \pm k+\sqrt{k^{2}+8}}{2}+1\right)_{(n)}\left(\frac{ \pm k-\sqrt{k^{2}+8}}{2}+1\right)_{(n)}}{(2)_{(n)} n !}(1-z)^{n} \log (1-z)+ \\
& (1-z) \sum_{n=1}^{\infty} \frac{\left(\frac{ \pm k+\sqrt{k^{2}+8}}{2}+1\right)_{(n)}\left(\frac{ \pm k-\sqrt{k^{2}+8}}{2}+1\right)_{(n)}}{(2)_{(n)} n !}(1-z)^{n} \\
& \left(\chi\left(\frac{ \pm k+\sqrt{k^{2}+8}}{2}+1+n\right)-\chi\left(\frac{ \pm k+\sqrt{k^{2}+8}}{2}+1\right)\right. \\
& +\chi\left(\frac{ \pm k-\sqrt{k^{2}+8}}{2}+1+n\right)-\chi\left(\frac{ \pm k-\sqrt{k^{2}+8}}{2}+1\right)-\chi(2+n)+ \\
& \chi(2)-\chi(n+1)+\chi(1)) .
\end{aligned}
$$

These two functions $f_{1(1)}$ and $f_{2(1)}$ are plotted in Figure A.1 for $z=\frac{r^{2}}{r_{c}^{2}}$. We see that the function $f_{1(1)}$ is an order of magnitude smaller than $f_{2(1)}$.

The third singularity of equation (A.4) is the singular point at infinity. The solutions for $z \rightarrow \infty$ are given by equations (15.5.7) and (15.5.8) in Abramowitz and Stegun (1964):

$$
\begin{aligned}
& f_{1(\infty)}(z)=z^{-a} F\left(a, a-c+1 ; a-b+1 ; \frac{1}{z}\right) \\
& f_{2(\infty)}(z)=z^{-b} F\left(b, b-c+1 ; b-a+1 ; \frac{1}{z}\right) .
\end{aligned}
$$

With the values of $a, b$, and $c$ for our problem (A.5) and (A.6) and with $p= \pm k$ these solutions are

$$
f_{1(\infty)}(z)=(z)^{\frac{ \pm k-\sqrt{k^{2}+8}}{2}} \sum_{n=0}^{\infty} \frac{\left(\frac{-k+\sqrt{k^{2}+8}}{2}\right)_{n},\left(\frac{k+\sqrt{k^{2}+8}}{2}\right)_{n}}{n !\left(\sqrt{k^{2}+8}+1\right)_{n}}(z)^{-n}
$$




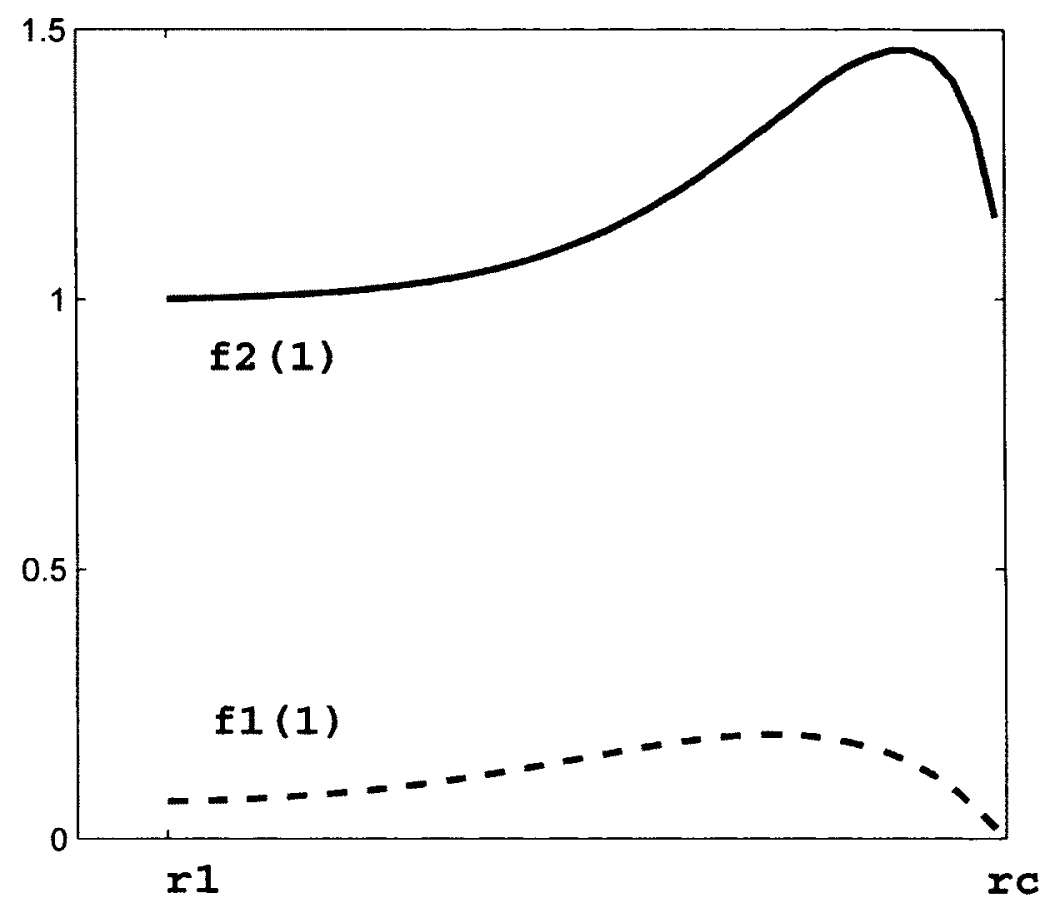

Figure A.1: The hypergeometric functions. The hypergeometric functions $f_{1(1)}\left(r^{2} / r_{c}^{2}\right)$ and $f_{2(1)}\left(r^{2} / r_{c}^{2}\right)$ in the interval $r_{1}<r<r_{c}, k=2, r_{1}=0.1 r_{c}$.

and

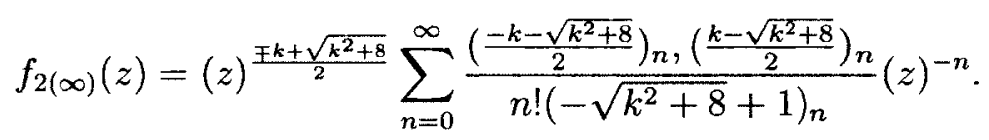

These solutions are valid for $|z|>1$. 


\section{Appendix B}

\section{Nonlinear evolution of the mean}

\section{flow}

The angular velocity of the background flow changes with time due to the momentum flux divergence at the critical layer (Montgomery and Kallenbach, 1997). To derive the equation for the time evolution of the angular velocity let us write the $\lambda$ component of the momentum equation (2.6) in terms of the streamfunction $\Psi(r, \lambda, t)$,

$$
\frac{\partial \Psi_{r}}{\partial t}=\left(-\frac{1}{r^{2}} \Psi_{\lambda} \frac{\partial}{\partial r}\left(r \Psi_{r}\right)+\frac{\Psi_{r}}{r} \Psi_{r \lambda}\right)+\frac{1}{\rho} \frac{\partial p}{\partial \lambda}
$$

We take the mean of each term in (B.1) in the azimuthal direction by integrating one with respect to $\lambda$ from 0 to $2 \pi$ and then dividing by $2 \pi$. This gives

$$
\frac{\partial \bar{\Psi}_{r}}{\partial t}=\overline{-\frac{1}{r^{2}} \Psi_{\lambda} \frac{\partial}{\partial r}\left(r \Psi_{r}\right)}+\overline{\frac{1}{r} \Psi_{r} \Psi_{r \lambda}}
$$

where the bar denotes the azimuthal mean. Using the fact that

$$
\frac{1}{r^{2}} \Psi_{\lambda} \frac{\partial}{\partial r}\left(r \Psi_{r}\right)=\frac{1}{r^{2}} \frac{\partial}{\partial r}\left(r \Psi_{\lambda} \Psi_{r}\right)-\frac{1}{r} \Psi_{r} \Psi_{\lambda r}
$$


we can rewrite equation (B.2) in the form

$$
\frac{\partial \bar{\Psi}_{r}}{\partial t}=\overline{-\frac{1}{r^{2}} \frac{\partial r \Psi_{r} \bar{\Psi}_{\lambda}}{\partial r}}+\overline{\frac{2}{r} \Psi_{r} \Psi_{r \lambda}}
$$

The mean of $\frac{1}{r} \Psi_{r} \Psi_{r \lambda}$ is

$$
\frac{1}{2 \pi} \int_{0}^{2 \pi} \frac{1}{r} \Psi_{r} \Psi_{r \lambda} d \lambda=\left.\frac{1}{2 \pi} \frac{1}{r}\left(\Psi_{r}\right)^{2}\right|_{0} ^{2 \pi}-\frac{1}{2 \pi} \int_{0}^{2 \pi} \frac{1}{r} \Psi_{r} \Psi_{r \lambda} d \lambda
$$

The first term on the right-hand side of (B.5) is zero. So

$$
2 \int_{0}^{2 \pi} \frac{1}{r} \Psi_{r} \Psi_{r \lambda} d \lambda=0
$$

and we can rewrite equation (B.4) in the form

$$
\frac{\partial \bar{\Psi}_{r}}{\partial t}=-\frac{1}{r^{2}} \frac{\partial r \Psi_{r} \Psi_{\lambda}}{\partial r}
$$

Recall from (3.49) that the streamfunction consists of 2 parts

$$
\Psi(r, \lambda, t)=\bar{\psi}(r)+\varepsilon \psi(r, \lambda, t)
$$

Let $\psi(r, \lambda, t)$ be the wave

$$
\psi(r, \lambda, t)=\phi(r, t) e^{i k \lambda}+\text { c.c. }=\phi(r, t) e^{i k \lambda}+\phi^{*}(r, t) e^{-i k \lambda}
$$

Derivatives of $\psi(r, \lambda, t)$ with respect to $r$ and $\lambda$ are

$$
\psi_{r}(r, \lambda, t)=\phi_{r}(r, t) e^{i k \lambda}+\phi_{r}^{*}(r, t) e^{-i k \lambda}
$$


and

$$
\psi_{\lambda}(r, \lambda, t)=i k \phi(r, t) e^{i k \lambda}-i k \phi_{r}^{*}(r, t) e^{-i k \lambda} .
$$

Hence,

$$
\begin{array}{r}
\psi_{r} \psi_{\lambda}=\left(\phi_{r}(r, t) e^{i k \lambda}+\phi_{r}^{*}(r, t) e^{-i k \lambda}\right)\left(i k \phi(r, t) e^{i k \lambda}-i k \phi_{r}^{*}(r, t) e^{-i k \lambda}\right) \\
=i k\left(\phi_{r} \phi^{*}-\phi_{r}^{*} \phi\right)=-2 k \operatorname{Im}\left(\phi \phi_{r}^{*}\right)
\end{array}
$$

In section 6.4 we discussed the time evolution of the mean flow by representing the mean streamfunction as a sum

$$
\bar{\psi}_{\text {total }}(r, t)=\bar{\psi}(r)+\psi_{0}(r, t)
$$

where $\bar{\psi}(r)$ is the initial mean flow, and $\psi_{0}(r, t)$ is the time-dependent mean streamfunction induced by nonlinearity. The corresponding azimuthal velocity is

$$
\bar{v}_{t o t a l}(r, t)=\bar{v}(r)+v_{0}(r, t)
$$

where

$$
\bar{v}_{\text {total }}(r, t)=\frac{\partial \bar{\Psi}_{\text {total }}(r, t)}{\partial r} .
$$

So from (B.7) and (B.12) the equation for the evolution of the mean azimuthal velocity is

$$
\frac{\partial \bar{v}_{\text {total }}}{\partial t}=\varepsilon^{2} \frac{1}{r^{2}} \frac{\partial}{\partial r}\left(2 k r \operatorname{Im}\left(\phi \phi_{r}^{*}\right)\right)
$$

or, for the mean angular velocity,

$$
\frac{\partial \vec{\Omega}_{\text {total }}}{\partial t}=\varepsilon^{2} \frac{1}{r^{3}} \frac{\partial}{\partial r}\left(2 k r \operatorname{Im}\left(\phi \phi_{r}^{*}\right)\right) .
$$




\section{Appendix C}

\section{The phase shift for the steady solution}

Here we will show that the phase shift of the steady solution (4.59) in the critical layer is $-\pi$. The steady-state solution (4.59), which is valid in the interval $r_{1}<r<\sqrt{2} r_{c}$, $r \neq r_{c}$, or $\left|1-\frac{r^{2}}{r_{c}^{2}}\right|<1$, includes the term $\left(1-r^{2} / r_{c}^{2}\right) \log \left(1-r^{2} / r_{c}^{2}\right)$. This term is singular close to the critical radius where $r=r_{r}$. We observe that $\log \left(1-\frac{r^{2}}{r_{c}^{2}}\right)=$ $\log \left|1-\frac{r^{2}}{r_{c}^{2}}\right|+i \theta$, where $\theta$ is the argument of $\left(1\right.$ of $\left(1-\frac{r^{2}}{r_{c}^{2}}\right): r<r_{c}, \theta=0$. For $r>r_{c}, \theta= \pm \pi$, and following Miles (1961), we can determine the sign by considering the inverse Laplace transform in the time-dependent problem that was considered in chapter 5 .

We can write the logarithmic term in the time-dependent solution (5.26) in the $s$-plane in the form 


$$
\begin{aligned}
\log (1 & \left.-\gamma(s) r^{2}\right) \\
& =\log \left(1-\frac{i k \Omega_{0} \alpha r^{2}}{s+i k \Omega_{0}}\right)=\log \left(1-\frac{i k \Omega_{0} \alpha r^{2}\left(\operatorname{Re}(s)-i \operatorname{Im}(s)-i k \Omega_{0}\right)}{\operatorname{Re}(s)^{2}+\left(\operatorname{Im}(s)+k \Omega_{0}\right)^{2}}\right) \\
= & \log \left(\left[1+\frac{k \Omega_{0} \alpha r^{2}\left(\operatorname{Im}(s)+k \Omega_{0}\right)}{\operatorname{Re}(s)^{2}+\left(\operatorname{Im}(s)+k \Omega_{0}\right)^{2}}\right]-i\left[\frac{k \Omega_{0} \alpha r^{2} \operatorname{Re}(s)}{\operatorname{Re}(s)^{2}+\left(\operatorname{Im}(s)+k \Omega_{0}\right)^{2}}\right]\right) .
\end{aligned}
$$

Consider the complex-valued function

$$
\zeta(s)=\left[1+\frac{k \Omega_{0} \alpha r^{2}\left(\operatorname{Im}(s)+k \Omega_{0}\right)}{\operatorname{Re}(s)^{2}+\left(\operatorname{Im}(s)+k \Omega_{0}\right)^{2}}\right]-i\left[\frac{k \Omega_{0} \alpha r^{2} \operatorname{Re}(s)}{\operatorname{Re}(s)^{2}+\left(\operatorname{Im}(s)+k \Omega_{0}\right)^{2}}\right] .
$$

We note that $\operatorname{Re}(s)>0$ along the contour of integration $(a-i \infty, a+i \infty)$ since $a>0$. So $\operatorname{Im}(\zeta(s))<0$. This means that

$$
-\pi<\arg (\zeta)<0
$$

The steady solution corresponds to the pole $s=-i \omega$. We consider the limit $s \rightarrow-i \omega$, which means that $\operatorname{Re}(s) \rightarrow 0$ and $\operatorname{Im}(s) \rightarrow-\omega$. As $s \rightarrow-i \omega$,

$$
\zeta(s) \rightarrow 1-\frac{k \Omega_{0} \alpha r^{2}\left(-\omega+k \Omega_{0}\right)}{\left(-\omega+k \Omega_{0}\right)^{2}}=1-\frac{k \Omega_{0} \alpha r^{2}}{k \Omega_{0} \alpha r_{c}^{2}}=1-\frac{r^{2}}{r_{c}^{2}}
$$

This means that $\log (\zeta(s)) \rightarrow \log \left(1-\frac{r^{2}}{r_{c}^{2}}\right)$, which can be written as

$$
\log |\zeta(s)|+i \arg (\zeta(s)) \rightarrow \log \left|1-\frac{r^{2}}{r_{c}^{2}}\right|+i \arg \left(1-\frac{r^{2}}{r_{c}^{2}}\right)
$$

Taking this limit allows us to determine the correct sign of the argument of $\left(1-\frac{r^{2}}{r_{c}^{2}}\right)$ 
in the steady-state solution (4.59). We noted in chapter 4 that

$$
\arg \left(1-\frac{r^{2}}{r_{c}^{2}}\right)=\left\{\begin{array}{l}
0, \quad \text { if } r<r_{c} \\
\pm \pi=\theta, \quad \text { if } r>r_{c}
\end{array}\right.
$$

Since $-\pi<\arg (\zeta)<0$, we conclude that as $s \rightarrow-i \omega, \arg (\zeta(s)) \rightarrow 0$ if $r<r_{c}$ and $\arg (\zeta(s)) \rightarrow-\pi$ if $r>r_{c}$. This means that the phase shift $\theta=-\pi$. 


\section{Bibliography}

[1] Abramowitz, M., Stegun, I.A., 1964, Handbook of mathematical functions with formulas, graphs and mathematical tables, Nat. Bur. Stands, 1046 pp.

[2] Abarca, S.F., Corbosiero, K.L., 2011, Secondary eyewall formation in WRF simulations of Hurricanes Rita and Katrina(2005), Geophys. Res. Lett., 38, L07802, doi:10.1029/2011GL047015.

[3] Abdullah, A.J., 1966, The spiral bands of a hurricane: A possible dynamic explanation, J. Atmos. Sci., 23, 365-375.

[4] Booker, J.R., Bretherton, F.P., 1967, The critical layer for gravity waves in a shear flow, J. Fluid Mech., 27, 513-539.

[5] Brunet, G., Montgomery, T.M., 2002, Vortex Rossby waves on smooth circular vortices, Part 1, theory, Dyn. Atmos. Oceans, 35, 135-177.

[6] Caillol, P., 2012, Multiple vortices induced by a threedimensional critical layer in a rapidly rotating vortex, J. Appl. Math., 77, 282-292.

[7] Campbell, L.J., 2004, Wave mean-flow interactions in a forced Rossby wave packet, Stud. Appl. Math, 112, 39-85.

[8] Campbell, L.J., Maslowe, S.A., 1998, Forced Rossby wave packets in barotropic shear flows with critical layers, Dyn. Atmos. Oceans, 28, 9-37. 
[9] Dickinson, R.E., 1970, Development of a Rossby wave critical level, J. Atmos. Sci., 27, 627-633.

[10] Emanuel, K., 2003, Tropical cyclones, Annu. Rev. Earth Pl. Sc., 31, 75-104.

[11] Erdelyi, A., 1953, Higher Transcendental Functions, Mc Graw-Hill, New York, Volume 1, $302 \mathrm{pp}$.

[12] Fortner, L.E., 1958, Typhoon Sarah, 1956, B. Am. Meteorol. Soc. 30, 633639.

[13] Gill, A.E., 1982, Atmosphere-ocean dynamics, Academic Press, 662 pp.

[14] Guinn, T.A., Shubert, W.H., 1993, Hurricane spiral bands, J. Atmos. Sci., 50, $3380-3403$

[15] Hawkins, J.D., Helveston, M., 2008, Tropical cyclone multiple eyewall characteristics, 28th Conference of Hurricanes and Tropical Meteorology, Amer. Meteor. Soc., Orlando, FL, 28 April - 2 May.

[16] Hendricks, E.A., Schubert, W.H., Fulton, S.R., McNoldy, B.D., 2010, Spontaneous-adjustment emission of inertia-gravity waves by unsteady vortical motion in the hurricane core, Q. J. R. Meteorol. Soc., 136, 537-548.

[17] Holton, J.R., 1992, An introduction to dynamic meteorology, third edition, Elsevier Academic Press, 511 pp.

[18] Holton, J.R., Lindzen, R.S., 1972, An updated theory for the Quasi-Biennial cycle of the tropical stratosphere, J. Atmos. Sci., 29, 1076-1080.

[19] Houze Jr., R.A., Shuyi, S.C., Bradley, F.S., Wen-Chau, L., Bell, M.M., 2007, Hurricane intensity and eyewall replacement, Science, 315, 1235-1239. 
[20] Irish, J.L., Resio, D.T., Ratcliff, J.J., 2008, The influence of storm size on hurricane surge, J. Phys. Oceanogr., 38, 2003-2013.

[21] Judt, F., Chen, S.S., 2010, Convectively generated potential vorticity in rainbands and formation of the secondary eyewall in Hurricane Rita of 2005, J. Atmos. Sci., 67, 3581-3599.

[22] Kossin, J.P., Sitkowski, M., 2009, An objective model for identifying secondary eyewall formation in hurricanes, Mon. Weather Rev., 137, 876-892.

[23] Kundu, P.K., Cohen, I.M., 2004, Fluid Mechanics, third edition, Elsevier Academic Press, 759 pp.

[24] Kurihara, Y., 1976, On the development of spiral bands in a tropical cyclone, J. Atmos. Sci., 33, 940-958.

[25] Kurihara, Y., Tuleya, R.E., Bender, M.A., 1998, The GFDL hurricane prediction system and its performance in the 1995 hurricane season, Mon. Weather Rev., 126, 1306-1322.

[26] Kuo, H.-C., Schubert, W.H., Tsai, C.-L., Kuo Y.F., 2008, Vortex interactions and barotropic aspects of concentric eyewall formation, Mon. Weather Rev., 136, 5183-5198.

[27] Landau, L.D., Lifshitz, E.M., 1953, Course of theoretical physics, Fluid Mechanics, Nauka, $630 \mathrm{pp}$.

[28] MacDonald, N.J., 1968, The evidence for the existence of Rossby-like waves in the hurricane vortex, Tellus, 20, 138-150.

[29] Maclay, K.S., DeMaria, M., Vonder Haar, T.H., 2008, Tropical Cyclone InnerCore Kinetic Energy Evolution, Mon. Wea. Rev., 136, 48824898. 
[30] Martinez, Y., Brunet, G., Yau, M.K., Wang, X, 2011, On the dynamics of concentric eyewall genesis: space-time empirical normal modes diagnosis, $J$. Atmos. Sci., 68, 457-476.

[31] Martinez, Y., Brunet, G., Yau, M.K., 2010a, On the dynamics of twodimensional hurricane-like vortex symmetrization, J. Atmos. Sci., 67, 35593580.

[32] Martinez, Y., Brunet, G., Yau, M.K., 2010b, On the dynamics of twodimensional hurricane-like concentric rings vortex formation, J. Atmos. Sci., 67, 3253-3268.

[33] Miles, J.W., 1961, On the stability of heterogeneous flows, J. Fluid Mech., 10, 496-508.

[34] Montgomery, M.T., Kallenbach, R.J., 1997, A theory of vortex Rossby-waves and its application to spiral bands and intensity changes in hurricanes, $Q . J . R$. Meteorol. Soc., 123, 435-465.

[35] Nong, S., Emanuel, K., 2003, A numerical study of the genesis of concentric eyewalls in hurricanes, Q. J. R. Meteorol. Soc., 129, 3323-3338.

[36] Ogawa, A., 1992, Vortex flow, CRC Press, $311 \mathrm{pp.}$

[37] Schecter, D.A., Montgomery, M.T., 2004. Damping and pumping of a vortex Rossby wave in a monotonic cyclone: Critical layer stirring versus inertiabuoyancy emission, Phys. Fluids, 16, 1334-1348.

[38] Sitkowski, M., Kossin, J.P., Rozoff, C.M., 2011, Intensity and structure changes during hurricane eyewall replacement cycles, Mon. Weather Rev., 139, 38293847 . 
[39] Stewartson, K., 1978. The evolution of the critical layer of a Rossby wave, Geophys. Astro. Fluid, 9, 185-200.

[40] Terwey, W.D., Montgomery, M.T., 2008. Secondary eyewall formation in two idealized, full-physics modeled hurricanes, J. Geophys. Res., 113, D12112, doi:10.1029/2007JD008897.

[41] Terwey, W.D., Abarca, S.F., Montgomery, M.T., 2012. Question on "Convective generated potential vorticity in rainbands and formation of the secondary eyewall in hurricane Rita of 2005", to appear in J. Atmos. Sci., early-online release, e-View, doi: http://dx.doi.org/10.1175/JAS-D-12-0151.1

[42] Warn, T., Warn, H., 1976. On the development of a Rossby wave critical level. J. Atmos. Sci., 33, 2021-2024.

[43] Warn, T., Warn, H., 1978. The evolution of a nonlinear critical level. Stud. Appl. Math., 59, 37-71.

[44] Willoughby, H.E., Black, P.G., 1996, Hurricane Andrew in Florida: dynamics of a disaster, B. Am. Meteorol. Soc., 77, 543-549.

[45] Willoughby, H.E., Clos, J.A., Shoreibah, M.G., 1982. Concentric eyewalls, secondary wind maxima, and the evolution of the hurricane vortex, J. Atmos. Sci., 39, 395-411.

[46] Willoughby, H.E., Jin, H.-L., Lord, S.J., Piotrowicz, J.M., 1984, Hurricane structure and evolution as simulated by an axisymmetric nonhydrostatic numerical model, J. Atmos. Sci., 41, 1169-1186.

[47] Willoughby, H.E., Shapiro, L.J., 1982, The response of balanced hurricanes to local sources of heat and momentum, J. Atmos. Sci., 39, 378-394. 